Supporting Information

\title{
Selective Formylation and Methylation of Amines Using Carbon Dioxide and Hydrosilane Catalyzed by Alkaline Metal Carbonates
}

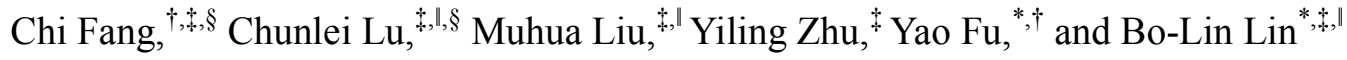 \\ ${ }^{\dagger}$ Department of Chemistry, University of Science and Technology of China, Hefei \\ 230026, China \\ ${ }^{*}$ School of Physical Science and Technology (SPST), ShanghaiTech University, \\ Shanghai 201210, China \\ "Shanghai Institute of Organic Chemistry, Chinese Academy of Sciences, Shanghai \\ 200032, China
}

Author Email Addresses: linb1@shanghaitech.edu.cn

fuyao@ustc.edu.cn 


\section{Table of Contents}

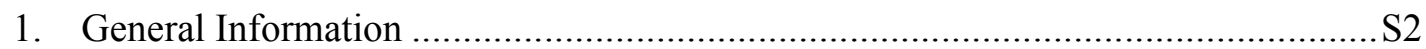

2. General procedure for the formylation of amines using carbon

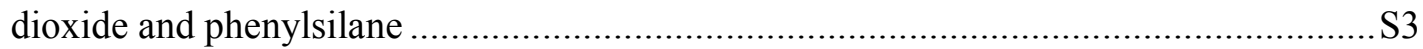

3. General procedure for the methylation of amines using carbon dioxide and

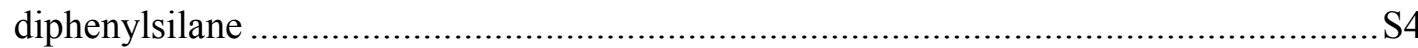

4. A "cesium effect" for $\mathrm{MACH}$ ………………………………………………....

5. Control experiments for the methylation of amines using carbon dioxide and

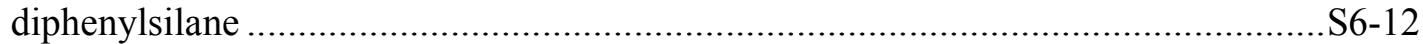

6. A proposed mechanism for the formylation ........................................................ 12

7. Procedure for stepwise hydrosilylation of $\mathrm{CO} 2$ and formylation of

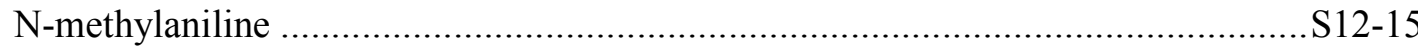

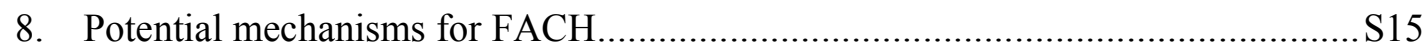

9. DFT calculations for the formation of silyl formate ………………….............. $16-18$

10. Reaction with $\mathrm{CsOCOH}$ or $\mathrm{KOSi}\left(\mathrm{CH}_{3}\right)_{3}$ or $\mathrm{CsOH}$ as the catalysts ........................... 19

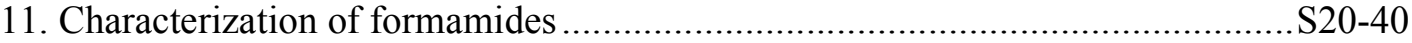

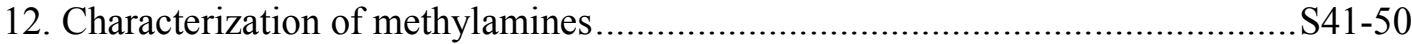

13. Characterization of N-methylated Cinacalcet ......................................................... 51

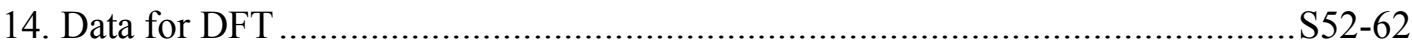

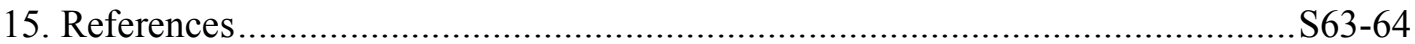




\section{General information}

\section{a. Materials}

All reactions were carried out in oven-dried Schlenk tubes under a carbon dioxide atmosphere (99.99\%). $\mathrm{Li}_{2} \mathrm{CO}_{3}$ was purchased from J\&K Scientific Ltd. $\mathrm{Na}_{2} \mathrm{CO}_{3}$ and $\mathrm{K}_{2} \mathrm{CO}_{3}$ were purchased from Sinopharm Chemical Reagent Co., Ltd. $\mathrm{Rb}_{2} \mathrm{CO}_{3}$ and $\mathrm{Cs}_{2} \mathrm{CO}_{3}$ were purchased from Aladdin Reagent Co., Ltd. Amines were purchased from Adamas, J\&K Scientific Ltd and Alfa Aesar. Silanes were purchased from J\&K Scientific Ltd. All solvents mentioned in this text were HPLC-grade and purchased from J\&K Scientific Ltd. All solvents were treated with solvent purification systems before use. The deuterated solvents $\left(\mathrm{CD}_{3} \mathrm{CN}, \mathrm{CDCl}_{3}\right)$ were purchased from J\&K Scientific Ltd. All chemicals were used as received unless otherwise stated.

\section{b. Analytical methods}

${ }^{1} \mathrm{H}$ and ${ }^{13} \mathrm{C}$ NMR spectra were recorded on Bruker Avance 500 spectrometer at ambient temperature in $\mathrm{CDCl}_{3}$ unless otherwise noted. Data for ${ }^{1} \mathrm{H}-\mathrm{NMR}$ are reported as follows: chemical shift ( $\delta \mathrm{ppm})$, multiplicity, integration, and coupling constant $(\mathrm{Hz})$. Data for ${ }^{13} \mathrm{C}-\mathrm{NMR}$ are reported in terms of chemical shift $(\delta \mathrm{ppm})$, multiplicity, and coupling constant (Hz). GC-MS analysis was performed on Thermo Scientific Ultimate 3000 GC-MS System. Gas chromatographic (GC) analyses were performed on a Jiedao GC-1620 series GC system equipped with a flame-ionization detector using anisole as an internal standard. Glove box (Vigor Tech USA) was used for the set-up of the reactions. Flash column chromatographic purification of the products was accomplished using forced-flow chromatography on Silica Gel (200-300 mesh). 


\section{General procedure for the formylation of amines using carbon dioxide and} phenylsilane

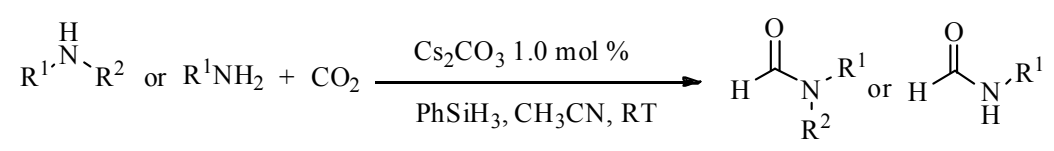

In a glovebox, $\mathrm{Cs}_{2} \mathrm{CO}_{3}(1 \mathrm{~mol} \%, 1.6 \mathrm{mg})$, phenylsilane $(0.5 \mathrm{mmol}, 65 \mu \mathrm{L})$, amine $(0.5 \mathrm{mmol})$ and $1 \mathrm{~mL}$ acetonitrile were added into a Schlenk tube equipped with a stir bar. The Schlenk tube was sealed in the glovebox, then the vessel was degassed by three freeze-pump-thaw cycles and then exposed to $c a$. 1 atmosphere of $\mathrm{CO}_{2}$ on the Schlenk line. The reaction mixture was stirred at room temperature for $12 \mathrm{~h}(450 \mathrm{rpm})$. The yields were determined by ${ }^{1} \mathrm{H}$ NMR spectroscopy of the crude reaction mixture using a specific amount of ferrocene as an internal standard. To isolate the products, the reaction mixture was diluted with EtOAc, filtered through a short silica gel column with EtOAc as the eluent. The resultant solution was concentrated and purified by silica gel column chromatography to give the corresponding formamides. 


\section{General procedure for the methylation of amines using carbon dioxide and}

\section{diphenylsilane}

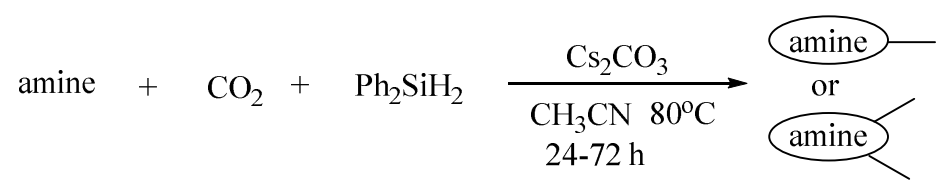

In a glovebox, $\mathrm{Cs}_{2} \mathrm{CO}_{3}(5-10 \mathrm{~mol} \%, 8 \mathrm{mg})$, diphenylsilane (1.5 mmol, $\left.281 \mu \mathrm{L}\right)$, amine $(0.25-0.5 \mathrm{mmol})$ and $1.6 \mathrm{~mL}$ acetonitrile were added into a Schlenk tube equipped with a stir bar. The Schlenk tube was sealed in the glovebox, then the vessel was degassed by three freeze-pump-thaw cycles and then sealed after being filled with ca. 1 atmosphere of $\mathrm{CO}_{2}$ on the Schlenk line. The reaction mixture was stirred at 80 ${ }^{\circ} \mathrm{C}$ for $24-72 \mathrm{~h}$ (450 rpm). The yields were determined by ${ }^{1} \mathrm{H}$ NMR spectroscopy of the crude reaction mixture using a specific amount of ferrocene as an internal standard. To determine the isolated yield of the methylated amines, the mixture was filtered, concentrated, and purified by silica gel column chromatography (petroleum ether) to give the corresponding methylated amines. The detailed numbers for the amount of amines and reaction times are shown below.

0.5 mmol: 1a, $1 \mathbf{n}$

0.25 mmol : 1b-1 m, 1o-1t

24h: 1a, 1n

48h: 1b, 1d, 1e, 1g, 1i, 11, 1m, 1s, $1 \mathrm{t}$

72h: 1c, 1f, 1h, 1j, 1k, 1o, 1p, 1q, 1r 


\section{A "cesium effect" for MACH}

Table S1. The methylation of $N$-methylaniline catalyzed by various carbonates. ${ }^{a}$

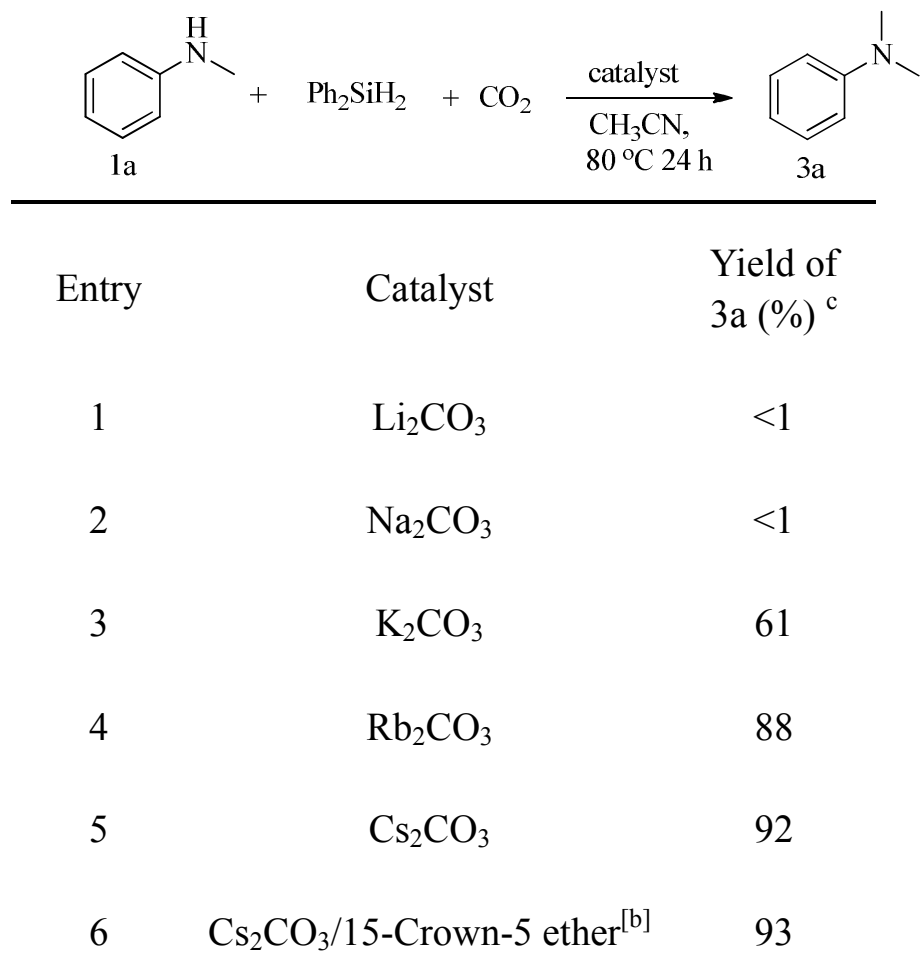

${ }^{a}$ Reaction conditions: $N$-methylaniline $(0.5 \mathrm{mmol}), \mathrm{Ph}_{2} \mathrm{SiH}_{2}(1.5 \mathrm{mmol})$, catalyst $(5.0$ mol \%), $\mathrm{CH}_{3} \mathrm{CN}$ (1.6 mL), $\mathrm{CO}_{2}$ (4 equiv), $80{ }^{\circ} \mathrm{C}, 24$ h. ${ }^{b} 15$-Crown-5 ether (10 mol \%). ${ }^{c}$ Yields were determined by ${ }^{1} \mathrm{H}$ NMR spectroscopy of the crude reaction mixture using ferrocene as the internal standard. 


\section{Control experiments for the methylation of amines using carbon dioxide and diphenylsilane}

\subsection{Reaction of $N, N$ '-diphenyl- $N$, $N$ '-dimethylurea (path A).}

In a glovebox, $\mathrm{Cs}_{2} \mathrm{CO}_{3}(10 \mathrm{~mol} \%, 8 \mathrm{mg})$, diphenylsilane $(1.5 \mathrm{mmol}, 281 \mu \mathrm{L})$, $N, N^{\prime}$-diphenyl- $N, N$ '-dimethylurea $(0.25 \mathrm{mmol}, 50 \mathrm{mg})$ and $1.6 \mathrm{~mL}$ acetonitrile were added into a Schlenk tube equipped with a stir bar. The Schlenk tube was sealed in the glovebox. Then the vessel was degassed by three freeze-pump-thaw cycles and sealed after being filled with $c a$. 1 atmosphere of $\mathrm{CO}_{2}$ on the Schlenk line. The mixture was stirred at $80^{\circ} \mathrm{C}$ for $24 \mathrm{~h}(450 \mathrm{rpm})$. Neither $N, N$-dimethylaniline nor $N$-methylaniline was detected by ${ }^{1}$ H NMR (Scheme S1).

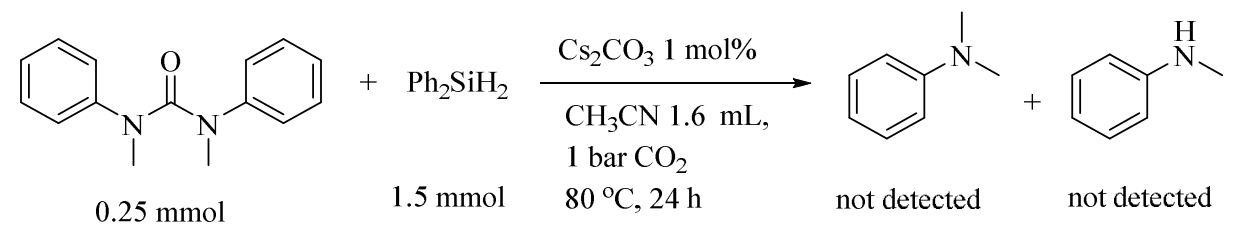

Scheme S1. Reaction between $N, N^{\prime}$-diphenyl- $N, N^{\prime}$-dimethylurea and diphenylsilane.

\subsection{Sequential formation of the formamide and the methylamine (path $B$, Scheme S2).}

In a glovebox, a $10 \mathrm{~mL}$ Schlenk tube equipped with a stir bar was charged with a magnetic stir bar, N-methylaniline ( $1 \mathrm{mmol}, 112 \mu \mathrm{L})$, phenylsilane $(1 \mathrm{mmol}, 130 \mu \mathrm{L})$, $\mathrm{Cs}_{2} \mathrm{CO}_{3}(5 \mathrm{~mol} \%, 16 \mathrm{mg})$, ferrocene $(0.2 \mathrm{mmol}, 37.2 \mathrm{mg}$, internal standard $)$ and $\mathrm{CD}_{3} \mathrm{CN}(1.5 \mathrm{~mL})$. The deuterated acetonitrile was dried with calcium hydride for $48 \mathrm{~h}$ and then distilled under an nitrogen atmosphere. A portion of the liquid mixture $(0.4$ $\mathrm{mL}$ ) was transferred into an NMR tube for ${ }^{1} \mathrm{H}$ NMR analysis (Figure S1). The Schlenk tube with the remaining mixture was degassed via three freeze-pump-thaw cycles and then exposed to $c a$. 1 atmosphere of $\mathrm{CO}_{2}$ on the Schlenk line. After stirring at room temperature for 12 hours, the $\mathrm{CO}_{2}$ in the tube was replaced with $\mathrm{N}_{2}$ on the Schlenk line. The Schlenk tube was then moved into the glovebox. And $0.4 \mathrm{~mL}$ reaction liquid was transferred into an NMR tube for ${ }^{1} \mathrm{H}$ NMR analysis (Figure S2). The formamide was detected. Then, the second equivalent of phenylsilane $(1 \mathrm{mmol}$, 
$130 \mu \mathrm{L}$ ) was added into the Schlenk tube and the resultant mixture was allowed to be stirred at $80{ }^{\circ} \mathrm{C}$ for $24 \mathrm{~h}$. Finally, a $0.4 \mathrm{~mL}$ of the reaction mixture was transferred into an NMR tube for ${ }^{1} \mathrm{H}$ NMR analysis (Figure S3). The methylamine was detected.

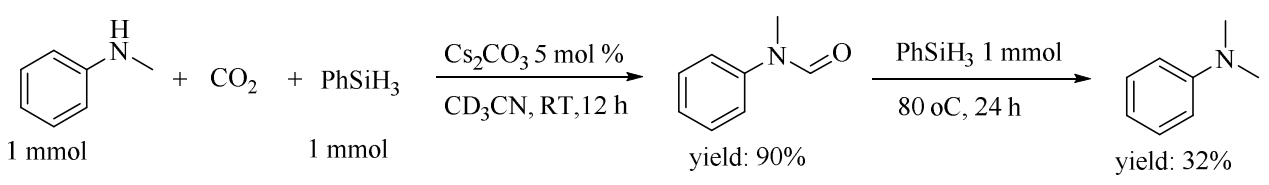

Scheme S2. Sequential formation of the formamide and the methylamine.

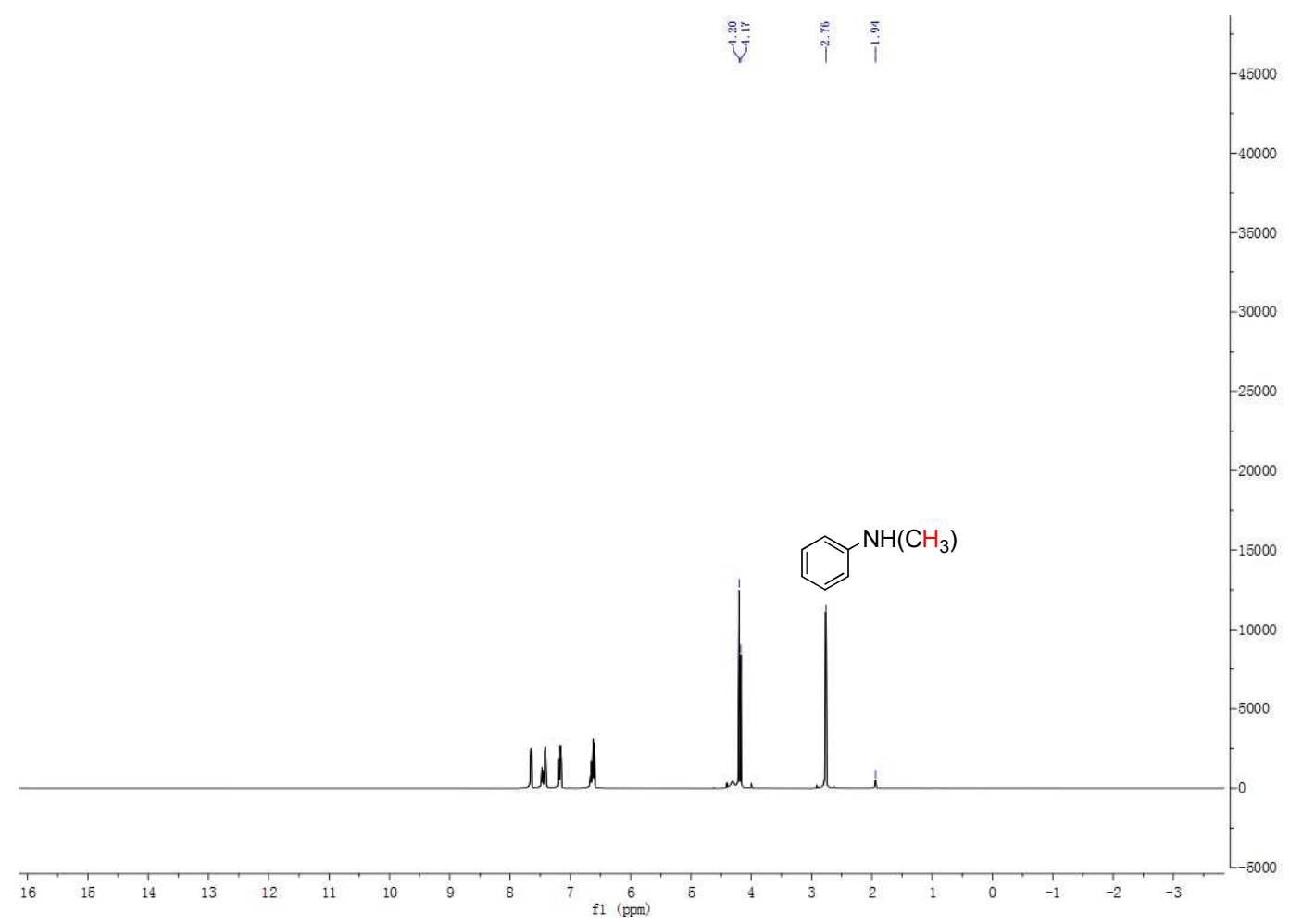

Figure S1. The ${ }^{1} \mathrm{H}$ NMR spectrum of the starting materials. 


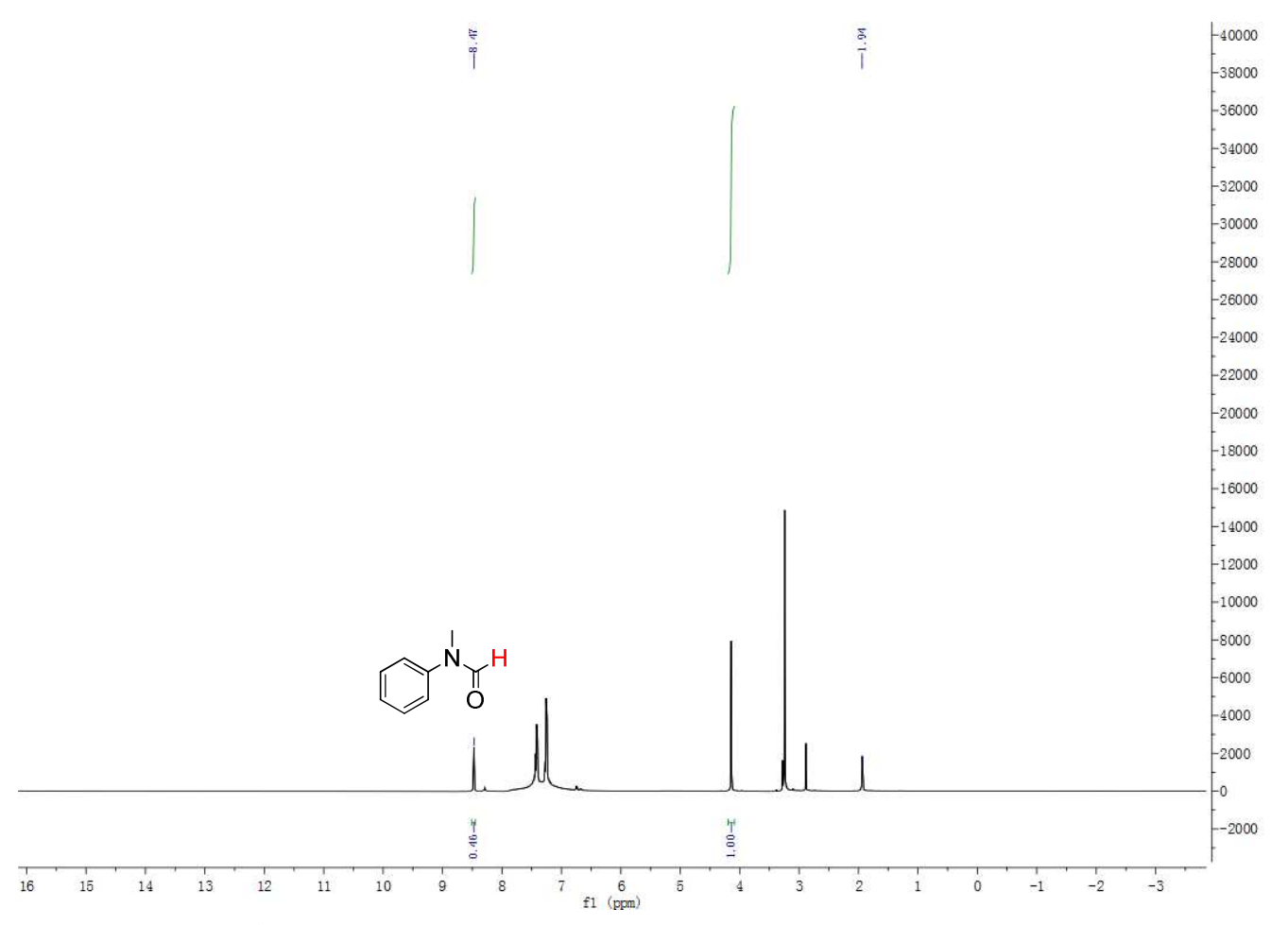

Figure S2. The ${ }^{1} \mathrm{H}$ NMR spectrum for the formylation product mixture.

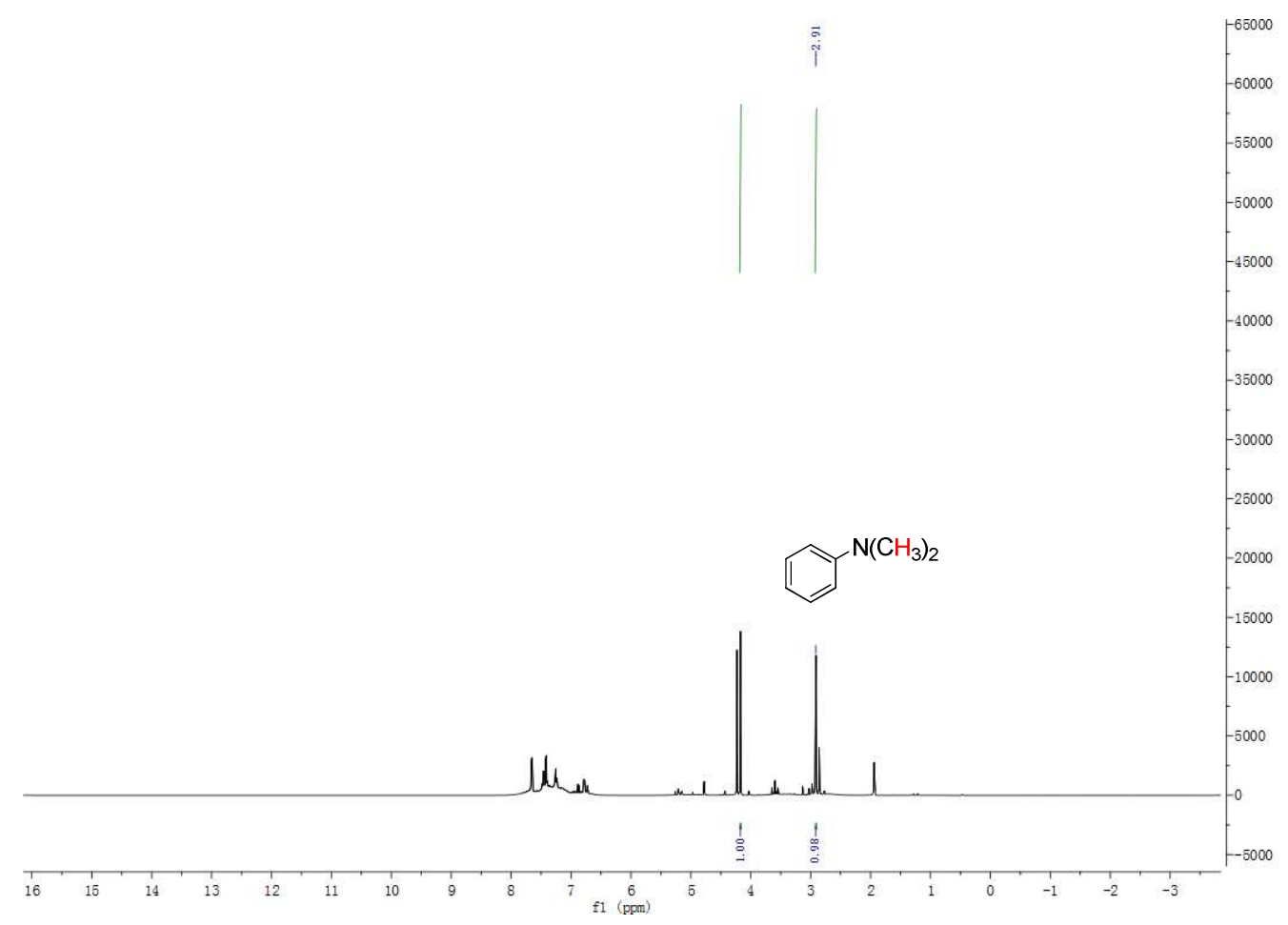

Figure S3. The ${ }^{1} \mathrm{H}$ NMR spectrum for the methylation product mixture. 
5.3 Sequential formation of the silyl methoxide and the methylamine (path $C$, Scheme S3).

In a glovebox, diphenylsilane $(2 \mathrm{mmol}, 375 \mu \mathrm{L}), \mathrm{Cs}_{2} \mathrm{CO}_{3}(10 \mathrm{~mol} \%, 8 \mathrm{mg})$, ferrocene $(0.2 \mathrm{mmol}, 37.2 \mathrm{mg})$ and $\mathrm{CD}_{3} \mathrm{CN}(2.0 \mathrm{~mL})$ were added into a Schlenk tube equipped with a stir bar. The deuterated acetonitrile was dried with calcium hydride for $48 \mathrm{~h}$ and then distilled under nitrogen atmosphere. A portion of the mixture $(0.4$ $\mathrm{mL}$ ) was transferred into an NMR tube for ${ }^{1} \mathrm{H}$ NMR analysis (Figure S4). The tube with the remaining mixture was degassed by three freeze-pump-thaw cycles and then sealed after being filled with $c a$. 1 atmosphere of $\mathrm{CO}_{2}$ on the Schlenk line. After stirring at $80{ }^{\circ} \mathrm{C}$ for 48 hours, the $\mathrm{CO}_{2}$ in the tube was replaced with $\mathrm{N}_{2}$ on the Schlenk line. In the glovebox, $0.4 \mathrm{~mL}$ reaction liquid was transferred into an NMR tube for ${ }^{1} \mathrm{H}$ NMR analysis (Figure S5). A significant amount of silyl methoxides (3-4 ppm) was observed. Then, $N$-methylaniline $(0.25 \mathrm{mmol}, 32.4 \mu \mathrm{L})$ was added into the reaction vessel. Another $0.4 \mathrm{~mL}$ liquid mixture was transferred into an NMR tube for ${ }^{1} \mathrm{H}$ NMR analysis upon mixing (Figure S6). No obvious change was observed. After stirring the remaining part at $80{ }^{\circ} \mathrm{C}$ for $24 \mathrm{~h}, 0.4 \mathrm{~mL}$ reaction mixture was transferred into an NMR tube for ${ }^{1} \mathrm{H}$ NMR analysis (Figure S7). The methylation product was not observed.

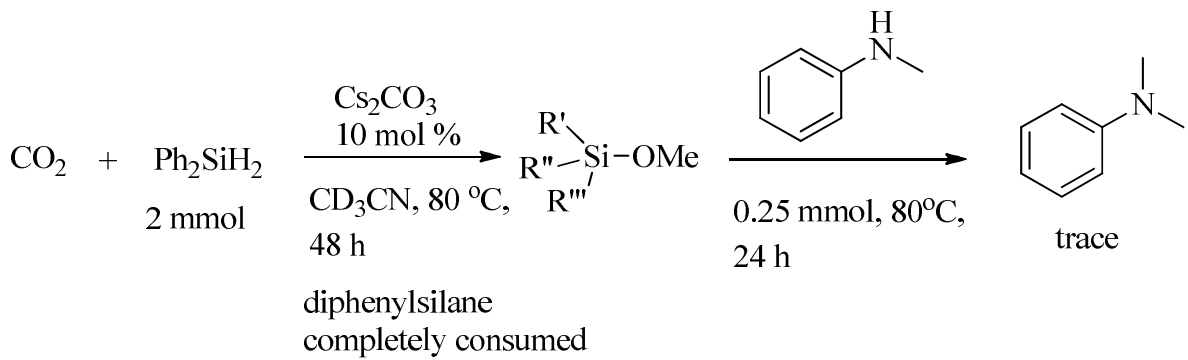

Scheme S3. Sequential formation of the silyl methoxide and the methylamine. 


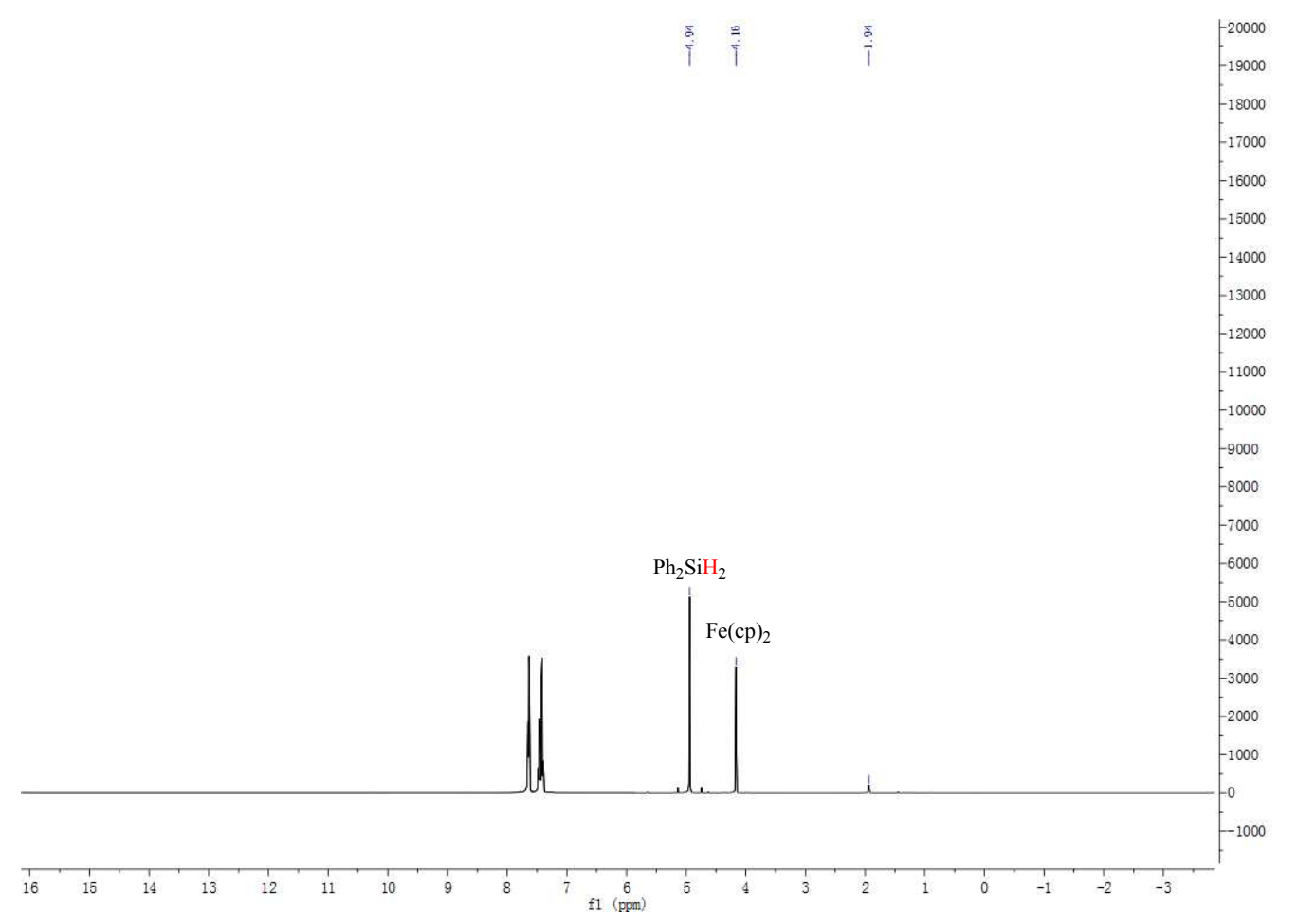

Figure S4. The ${ }^{1} \mathrm{H}$ NMR spectrum of the starting materials.

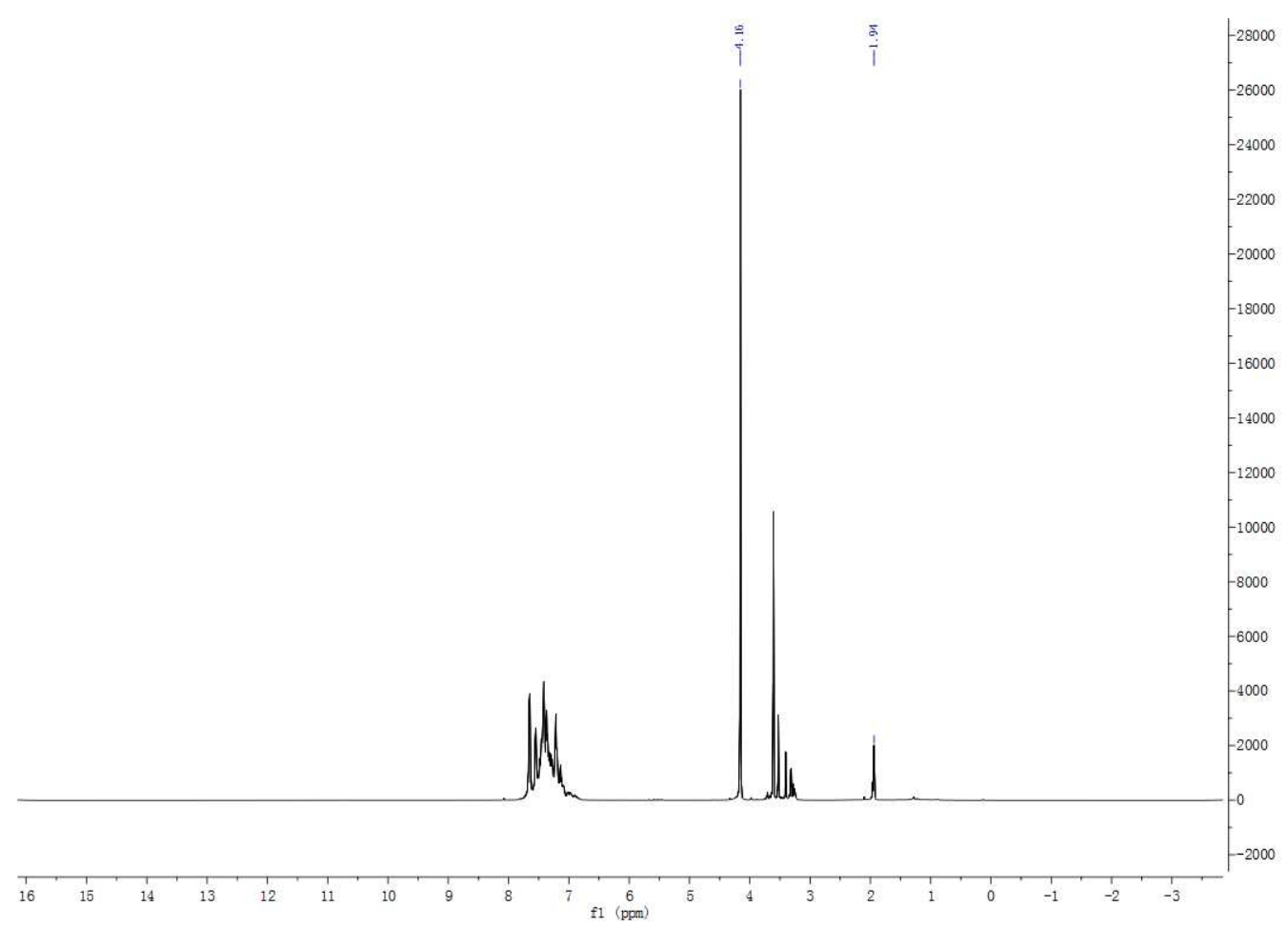

Figure S5. The ${ }^{1} \mathrm{H}$ NMR spectrum for the product mixture from hydrosilyation of $\mathrm{CO}_{2}$ in the absence of amines. The signals in the region of $c a$. 3.1-3.7 ppm are typical of silyl methoxides. ${ }^{1}$ 


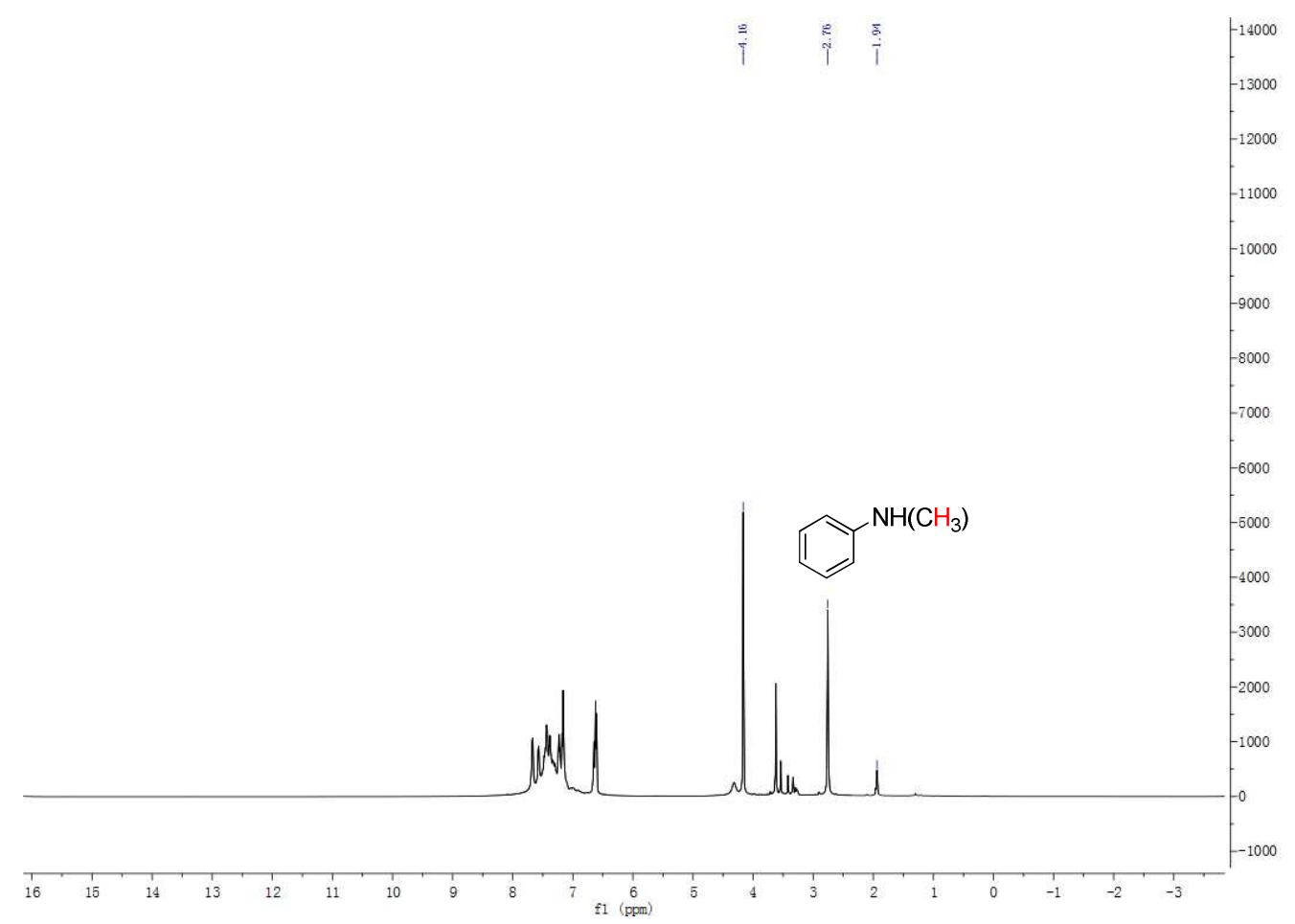

Figure S6. The ${ }^{1} \mathrm{H}$ NMR spectrum of the sample immediately after mixing the hydrosilyation products with 1a. No obvious change for the signals in the region of $c a$. 3.1-3.7 ppm were observed. 


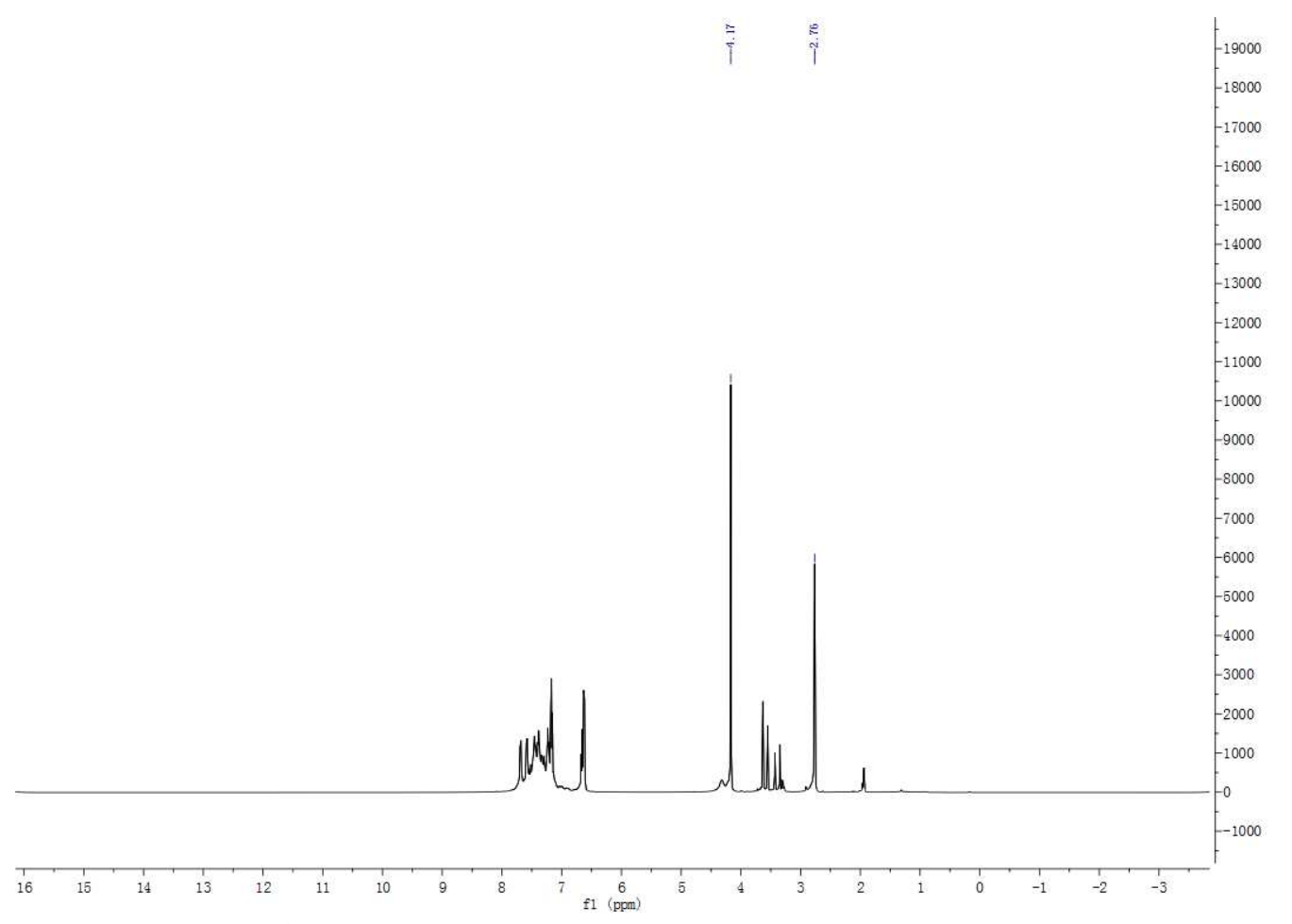

Figure S7. The ${ }^{1} \mathrm{H}$ NMR spectrum of the mixture of $\mathbf{1 a}$ and the hydrosilyation products after heating at $80{ }^{\circ} \mathrm{C}$ for 24 h. 3a was not observed.

\section{A proposed mechanism for the formylation.}

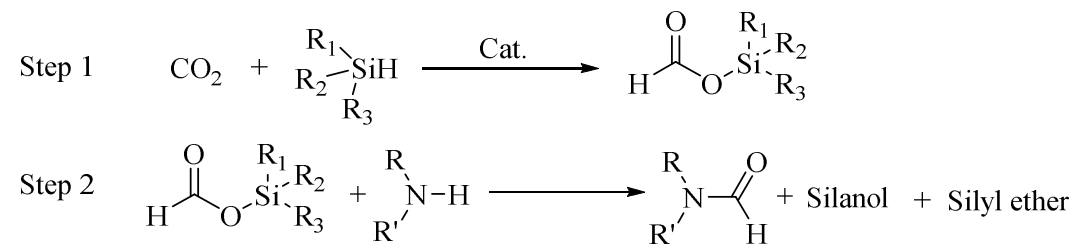

Scheme S4. A proposed mechanism for the formylation of amine with $\mathrm{CO}_{2}$ and hydrosilanes based on the experimental evidence reported in a related work. ${ }^{2}$

\section{Procedure for stepwise hydrosilylation of $\mathrm{CO}_{2}$ and formylation of $N$-methylaniline}

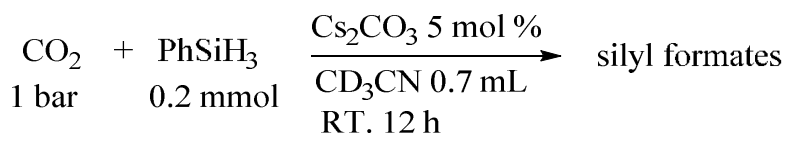

The deuterated acetonitrile was dried with calcium hydride for $48 \mathrm{~h}$ and then distilled under an nitrogen atmosphere. In a glovebox, $\mathrm{Cs}_{2} \mathrm{CO}_{3}(5 \mathrm{~mol} \%, 3.2 \mathrm{mg})$, phenylsilane $(0.2 \mathrm{mmol}, 26 \mu \mathrm{L})$ and $0.7 \mathrm{~mL}$ deuterated acetonitrile were added into a Schlenk tube equipped with a stir bar. The Schlenk tube was sealed in the glovebox. 
Then the vessel was degassed by three freeze-pump-thaw cycles and filled with $\mathrm{CO}_{2}$ via the Schlenk line. The reaction mixture was stirred at room temperature for $12 \mathrm{~h}$, transferred into an NMR tube in the golvebox, and then analyzed by ${ }^{1} \mathrm{H}$ NMR and ${ }^{13} \mathrm{C}$ NMR spectroscopies (Figure S8, S9). Subsequently, 0.2 mmol 1a was added into the NMR tube in the glovebox. The mixture was analyzed by ${ }^{1} \mathrm{H}$ NMR and ${ }^{13} \mathrm{C}$ NMR spectroscopies after 10 minutes and 10 hours at room temperature, respectively (Figures S10, S11). No apparent difference was observed for the mixture between the spectra of the two different time points. The GC-MS data further confirmed the formation of $N$-formanilide (Figure S12).

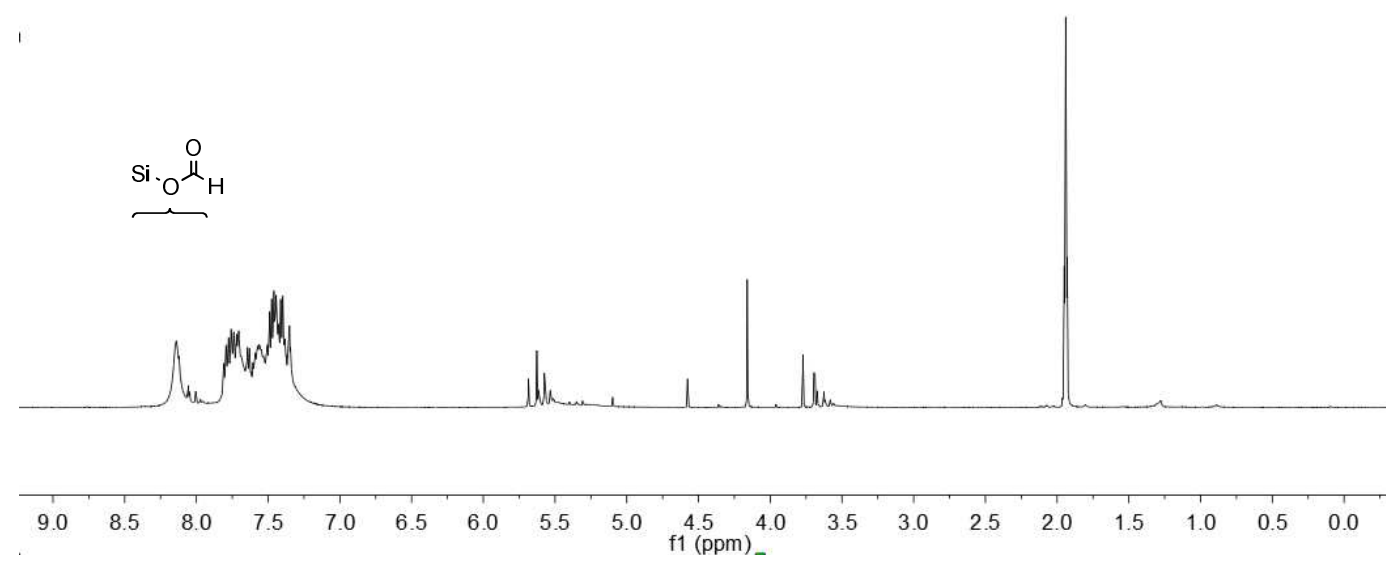

Figure S8. The ${ }^{1} \mathrm{H}$ NMR spectrum of the reaction mixture with $\mathrm{CO}_{2}$, phenylsilane and $\mathrm{Cs}_{2} \mathrm{CO}_{3}$ (5 mol\% of phenylsilane) in $\mathrm{CD}_{3} \mathrm{CN}$ after $12 \mathrm{~h}$. The broad signal at ca. 8.2 ppm is typical of silyl formates. The signal was consistent with the experimental results of Ying and Kinjo. ${ }^{1 \mathrm{a}, 2}$ 


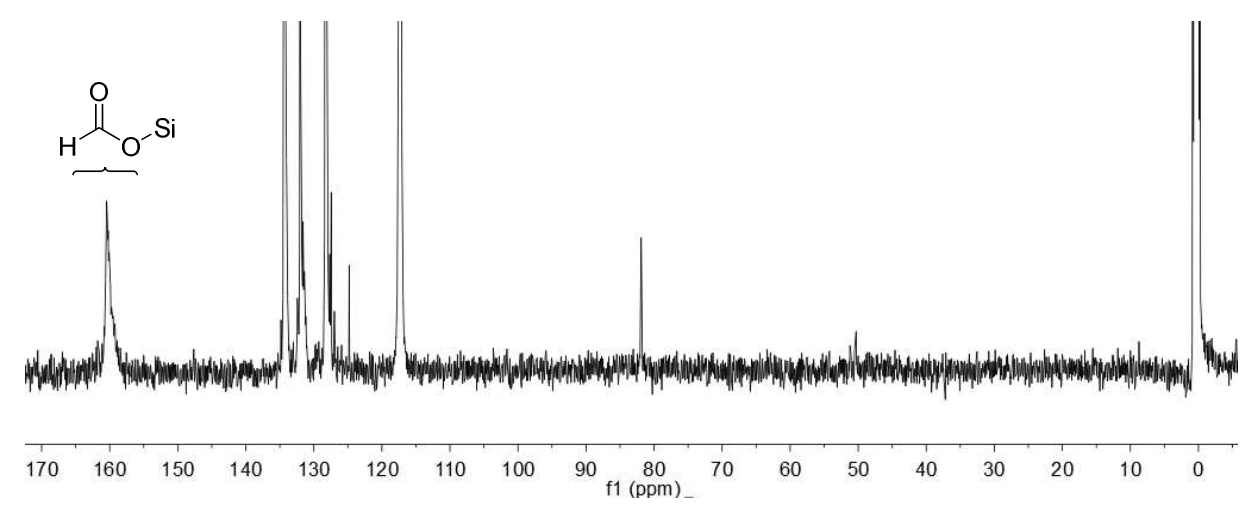

Figure S9. The ${ }^{13} \mathrm{C}$ NMR spectrum of the reaction mixture with $\mathrm{CO}_{2}$, phenylsilane and $\mathrm{Cs}_{2} \mathrm{CO}_{3}$ (5 mol \% of phenylsilane) in $\mathrm{CD}_{3} \mathrm{CN}$ after $12 \mathrm{~h}$. The signal at $c a .160$ ppm confirmed the formation of silyl formates. The signal was consistent with the experimental datas of Ying, Kinjo and Baba. ${ }^{1 \mathrm{a}, 1 \mathrm{~b}, 1 \mathrm{c}, 2}$

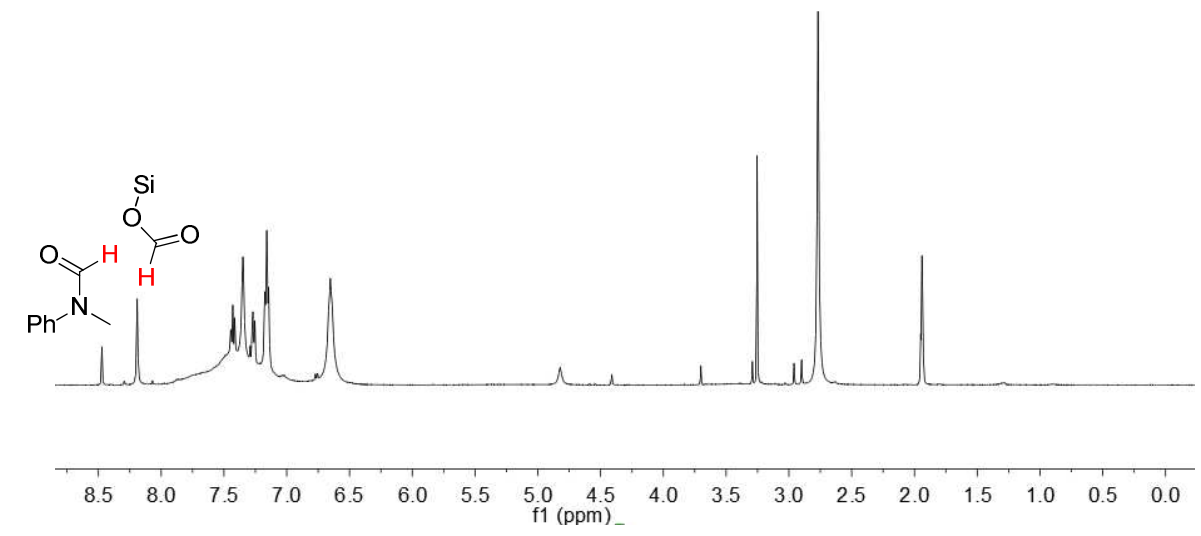

Figure S10. The ${ }^{1} \mathrm{H}$ NMR spectrum of the reaction mixture after the addition of 1 equiv $N$-methylaniline indicated the formation of $\mathbf{2 a}$.

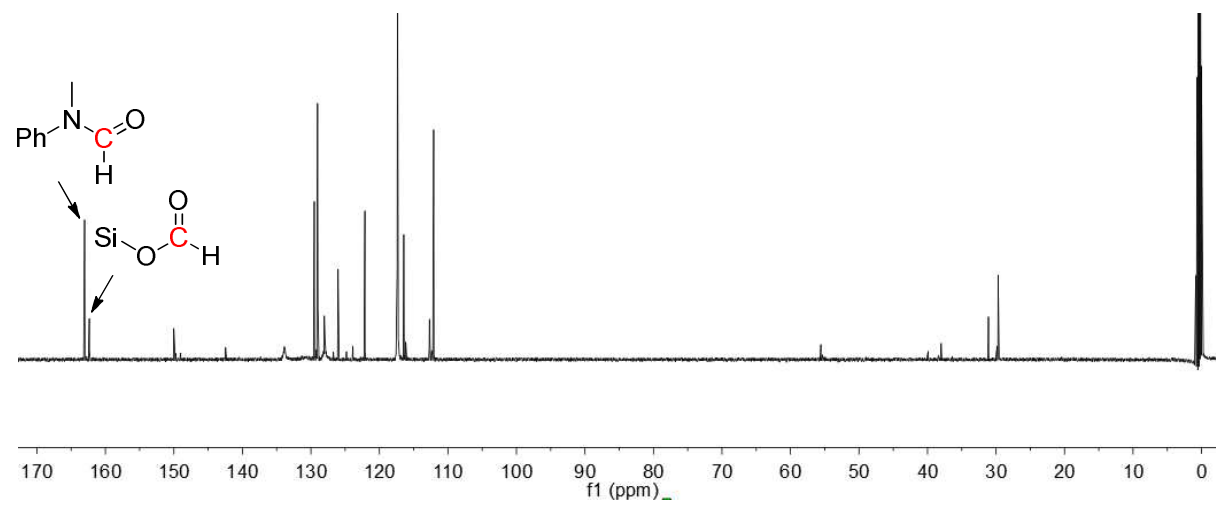

Figure S11. The ${ }^{13} \mathrm{C}$ NMR spectrum of the reaction mixture after the addition of 1 equiv $N$-methylaniline confirmed the formation of $\mathbf{2 a}$. 


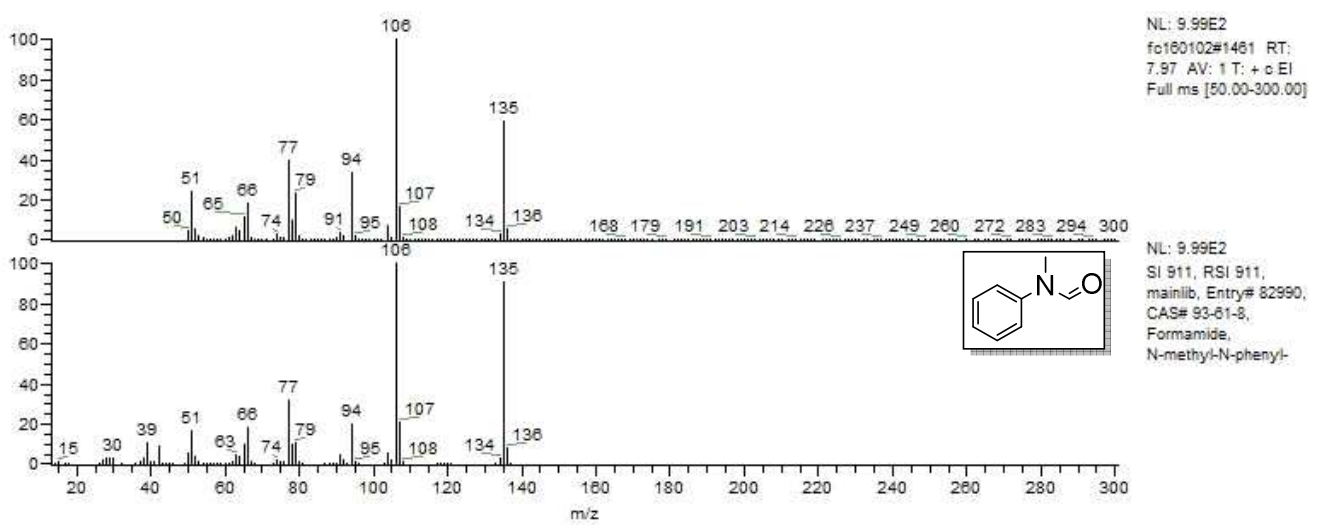

Figure S12. GC-MS data further confirmed the formation of 2a (upper: data from the reaction mixture; lower: authentic sample).

\section{Potential mechanisms for FACH.}
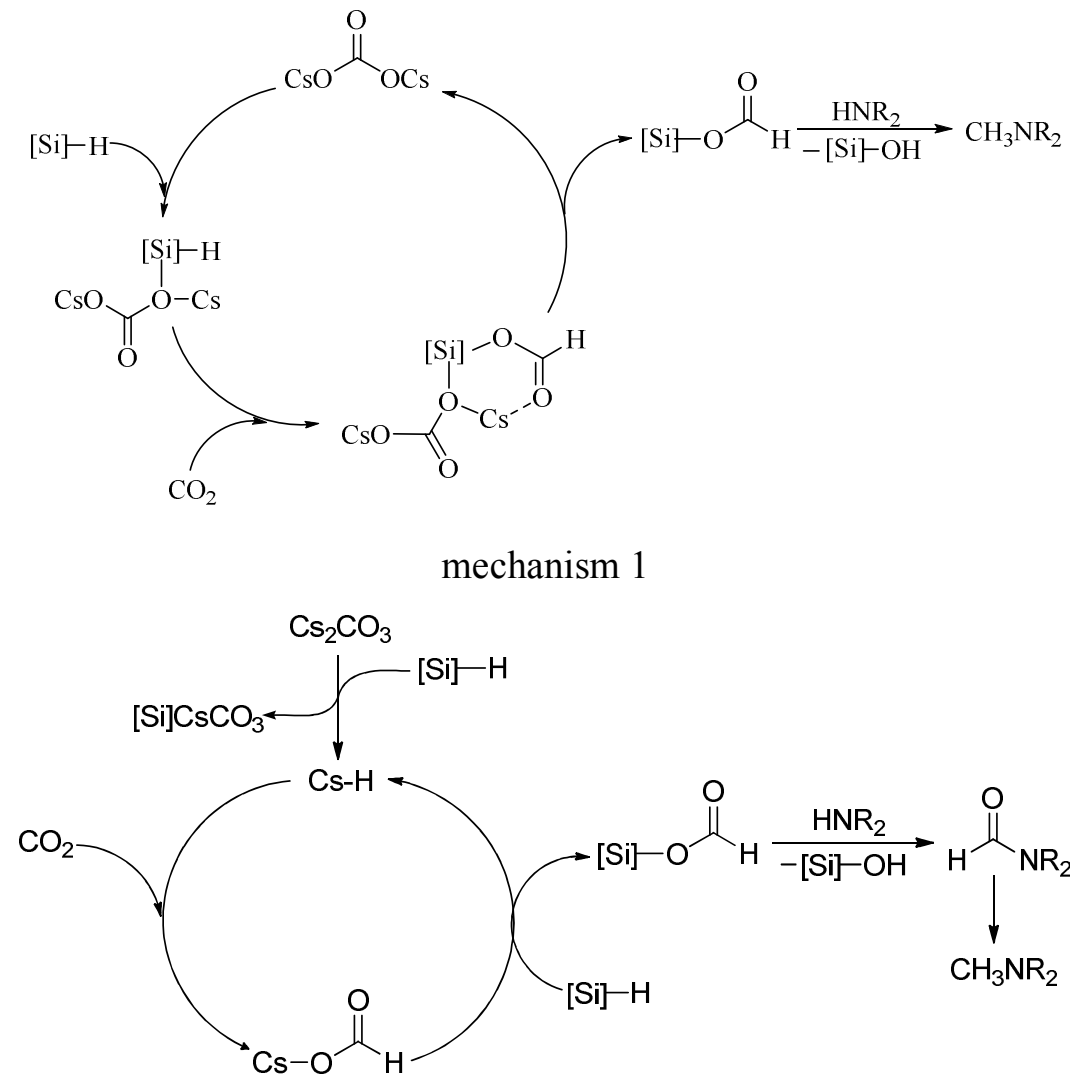

mechanism 2

Figure S13. Potential mechanisms for FACH. The key difference between the two mechanisms is whether the hydrogen transfers from $[\mathrm{Si}-\mathrm{H}]$ to the cesium cation or not. 


\section{DFT calculations for the formation of silyl formate. ${ }^{24}$}

In order to obtain insight into the proposed mechanisms, DFT calculations were employed using the Gaussian 09 program package. The geometry optimizations of all structures were performed at the b3lyp level of theory with the $6-31+G(d)$ basis set used for $\mathrm{H}, \mathrm{C}, \mathrm{N}, \mathrm{O}$, and $\mathrm{Si}$ atoms and lanl2dz basis set for $\mathrm{Cs}$ atom.

To ensure each structure was a true minimum, vibration frequencies were computed. Equilibrium geometries were characterized by the absence of imaginary frequency. The calculated vibration motion of the only one imaginary frequency in each transition structure illustrated the apparent transformation from the reactant to the product.

Subsequent calculations of the solvent effect of acetonitrile were conducted with the SMD model using gas phase optimized structures.

The energies reported in this paper are resulted from relative Gibbs free energies calculated at $298 \mathrm{~K}$ in solution and in the gas phase. Three different pathways (1, 2 and 3, Fig. S14) were identified computationally. Neither path 1 nor path 2 involves the transfer of hydrogen from $\mathrm{Si}-\mathrm{H}$ to $\mathrm{Cs}$, while $\mathrm{Cs}-\mathrm{H}$ is formed in path 3 . Whether the transferring hydrogen is cis- or trans- to the carbonate differentiates path 1 and path 2 . Although the cis- path (path 2) involves a six-membered ring that appears to stabilize the transition state through intramolecular Lewis acid-base interaction between the $\mathrm{Cs}^{+}$and the partially reduced $\mathrm{CO}_{2}$, path 1 shows a significantly lower activation barrier presumably due to the trans- influence of the carbonate onto the transferring hydrogen. The reaction energy of the last step of path 3 gives the lower limit of the overall activation, $31.2 \mathrm{kcal} \mathrm{mol}^{-1}$, significantly larger than both paths 1 and 2 . It should be noted that the imaginary varibational mode of TS-1 points to the formation of the $\mathrm{C}-\mathrm{H}$ bond of the formate. In principle, there should be an additional process corresponding to the formation of $\mathrm{Si}-\mathrm{O}$ bond of $\mathrm{Si}-2$ after that. However, all efforts to locate the transition state for such process failed and more calculations are still ongoing. 

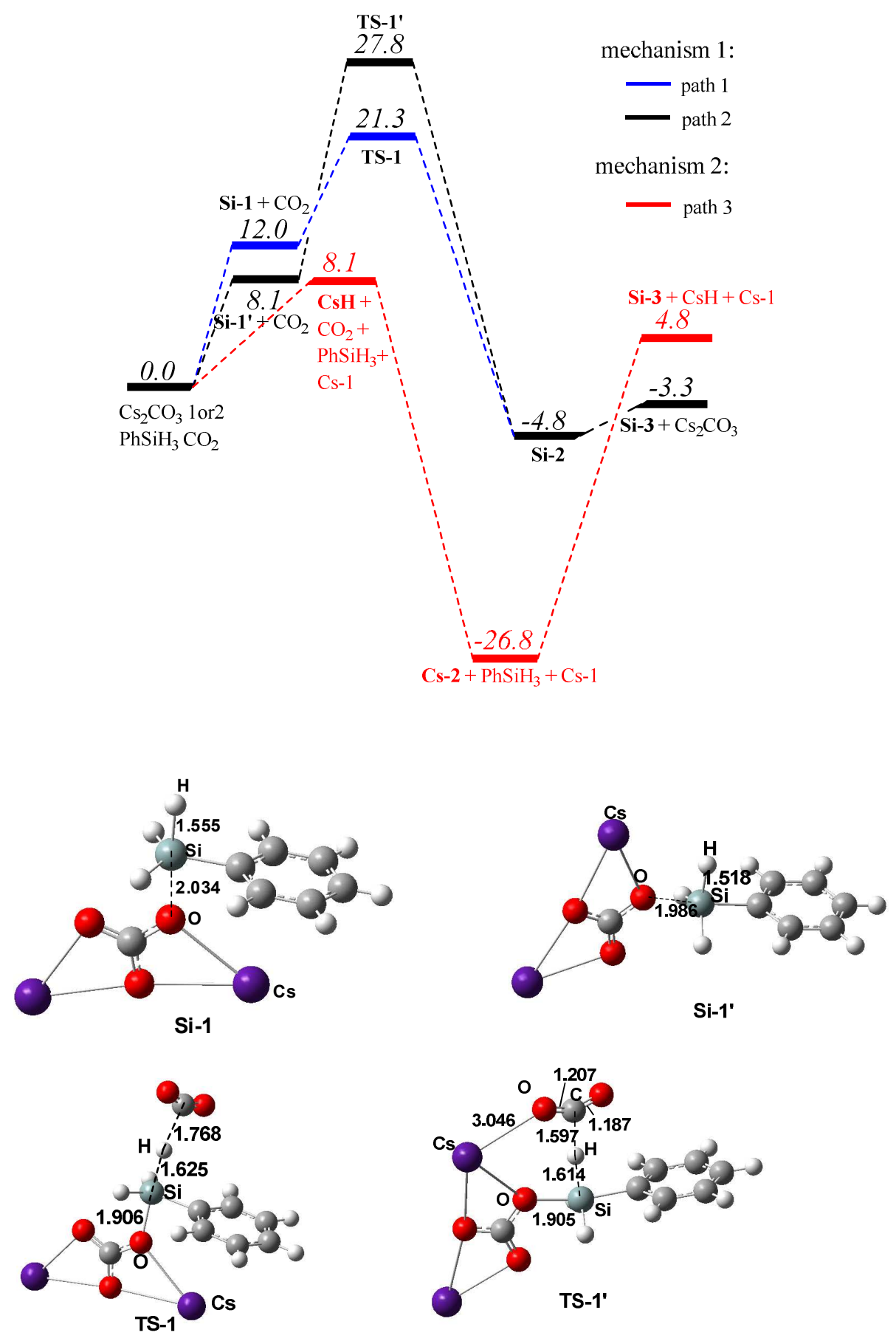

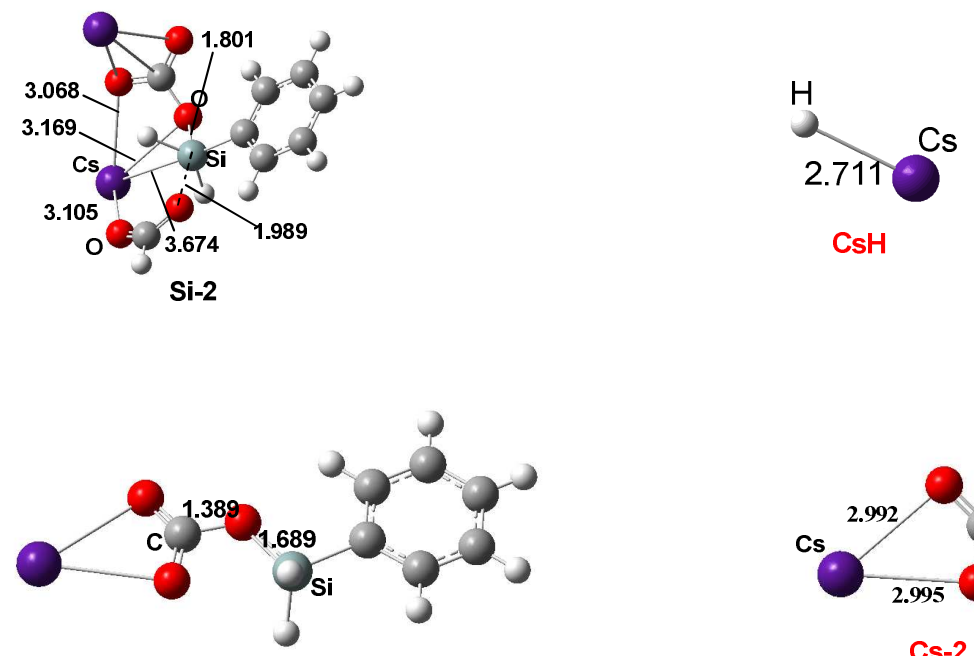

Cs-1
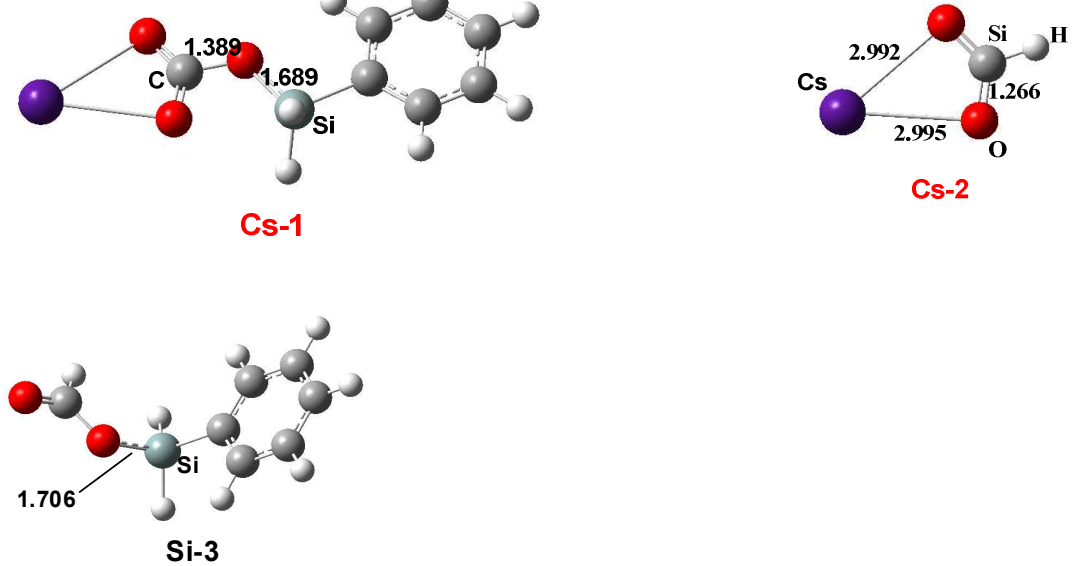

Figure S14. Free-energy profile (in $\mathrm{kcal} / \mathrm{mol}$ ) for silyl formate formation step and optimized structures of some important stationary points, along with key bond lengths (in $\AA$ ).

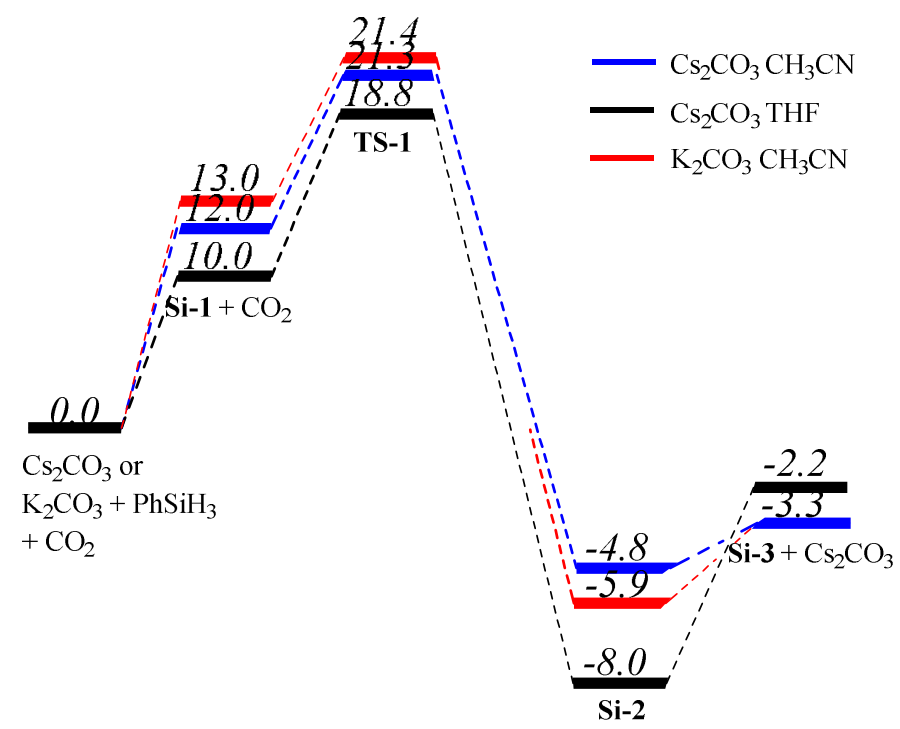

Figure S15. Free-energy profile (in $\mathrm{kcal} / \mathrm{mol}$ ) for the influence of different cations and solvents. 


\section{Reaction with $\mathrm{CsOCOH}$ or $\mathrm{KOSi}\left(\mathrm{CH}_{3}\right)_{3}$ or $\mathrm{CsOH}$ as the catalysts.}

In a glovebox, catalyst $(5 \mathrm{~mol} \%)$, phenylsilane $(0.5 \mathrm{mmol}, 65 \mu \mathrm{L}), \mathbf{1 a}(0.5 \mathrm{mmol})$ and $1 \mathrm{~mL}$ acetonitrile were added into a Schlenk tube equipped with a stir bar. The Schlenk tube was sealed in the glovebox, then the vessel was degassed by three freeze-pump-thaw cycles and then exposed to $c a .1$ atmosphere of $\mathrm{CO}_{2}$ on the Schlenk line. The reaction mixture was stirred at room temperature for $12 \mathrm{~h}(450 \mathrm{rpm})$. The

yields were determined by ${ }^{1} \mathrm{H}$ NMR spectroscopy of the crude reaction mixture using a specific amount ferrocene as an internal standard. The relatively lower yields compared to $\mathrm{Cs}_{2} \mathrm{CO}_{3}$ suggest that the latter might be a more likely active catalytic species (Scheme S5).

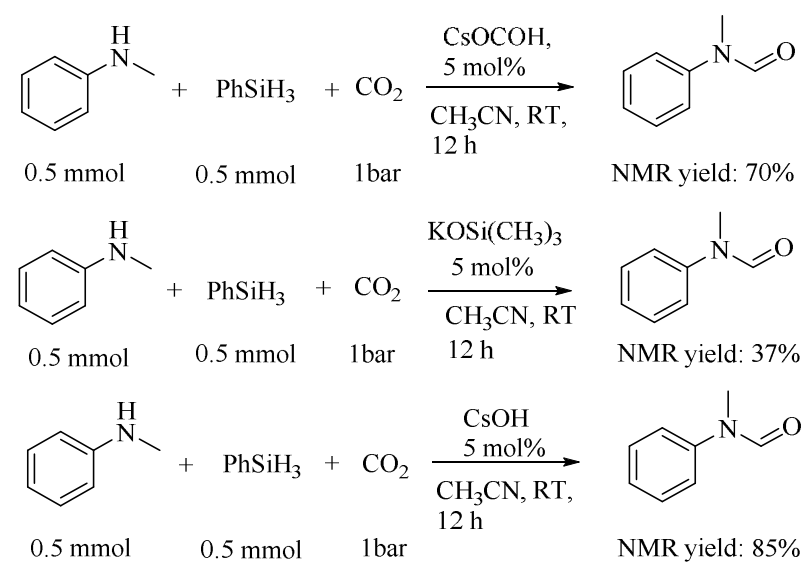

Scheme S5. The formylation of $\mathbf{1 a}$ using $\mathrm{CO}_{2}$ and phenylsilane catalyzed by cesium formate, a hypothetical intermediate in pathway 1 as well as two bases, i.e. $\mathrm{KOSi}\left(\mathrm{CH}_{3}\right)_{3}$ and $\mathrm{CsOH}$, that might be formed in the catalytic system (Figure $\mathbf{S 1 3}$ ). 


\section{Characterization of formamides}

The product for $\mathbf{2 e}$ in Table $\mathbf{2}$ was identified through comparison with commercial sample (J\&K Scientific Ltd). ${ }^{1} \mathrm{H}$ and ${ }^{13} \mathrm{C}$ NMR data for the products corresponding to $\mathbf{2} \mathbf{a}^{2}, \mathbf{2} \mathbf{b}^{3}, \mathbf{2} \mathbf{c}^{4}, \mathbf{2} \mathbf{d}^{5}, \mathbf{2} \mathbf{f}^{3}, \mathbf{2} \mathbf{g}^{6}, \mathbf{2} \mathbf{h}^{6}, \mathbf{2} \mathbf{i}^{2}, \mathbf{2} \mathbf{j}^{6}, \mathbf{2} \mathbf{k}^{7}, \mathbf{2} \mathbf{l}^{8}, \mathbf{2} \mathbf{m}^{7}, \mathbf{2} \mathbf{n}^{9}, \mathbf{2} \mathbf{o}^{10}, \mathbf{2} \mathbf{p}^{6}, \mathbf{2} \mathbf{q}^{2}, \mathbf{2} \mathbf{r}^{11}, \mathbf{2} \mathbf{s}^{8}$, $\mathbf{2} \mathbf{t}^{8}, \mathbf{2} \mathbf{u}^{12}, \mathbf{2} \mathbf{v}^{13}, \mathbf{2} \mathbf{w}^{14}$ and $\mathbf{2} \mathbf{x}^{15}$ in Table 2 were in accordance with those reported in the literatures.

${ }^{1} \mathrm{H}$ NMR data for the products with isolated yields in Table 2<smiles>O=CN1CC2CCCCC21</smiles>

$\mathrm{N}$-methyl- $\mathrm{N}$-phenylformamide (2a) $\mathbf{( 9 0 \% )}$ ) about $96: 4$ mixture of rotamers. Major rotamer: ${ }^{1} \mathbf{H}$ NMR $\left(500 \mathrm{MHz}, \mathrm{CDCl}_{3}\right) \delta=8.49(\mathrm{~s}, 1 \mathrm{H}, \mathrm{CHO}), 7.44-7.40(\mathrm{~m}, 2 \mathrm{H}$, $\mathrm{ArH}), 7.31-7.28(\mathrm{~m}, 1 \mathrm{H}, \mathrm{ArH}), 7.20-7.18(\mathrm{~m}, 2 \mathrm{H}, \mathrm{ArH}), 3.34$ (s, 3H, $\left.\mathrm{CH}_{3}\right)$.<smiles>O=CN(CC1CC2CCC1C2)c1ccccc1</smiles>

$\boldsymbol{N}$-methyl- $\mathbf{N}$-(p-tolyl)formamide (2g) $\mathbf{( 8 0 \% )}$ about 95:5 mixture of rotamers. Major rotamer: ${ }^{1} \mathbf{H}$ NMR $\left(500 \mathrm{MHz}, \mathrm{CDCl}_{3}\right) \delta=8.43(\mathrm{~s}, 1 \mathrm{H}, \mathrm{CHO}), 7.22(\mathrm{~d}, J=8.0 \mathrm{~Hz}, 2 \mathrm{H}$, $\mathrm{ArH}), 7.07$ (m, 2H, $\mathrm{ArH}), 3.30\left(\mathrm{~s}, 3 \mathrm{H}, \mathrm{N}-\mathrm{CH}_{3}\right), 2.38$ (s, 3H, $\left.\mathrm{Ar}-\mathrm{CH}_{3}\right)$.<smiles>CN(C=O)c1cccc(Cl)c1</smiles>

$\boldsymbol{N}$-(3-chlorophenyl)- $\boldsymbol{N}$-methylformamide (2h) (83\%) about 93:7 mixture of rotamers. Major rotamer: ${ }^{1} \mathbf{H}$ NMR $\left(500 \mathrm{MHz}, \mathrm{CDCl}_{3}\right) \delta=8.48(\mathrm{~s}, 1 \mathrm{H}, \mathrm{CHO}), 7.35(\mathrm{t}$, $J=8.0 \mathrm{~Hz}, 1 \mathrm{H}, A r H), 7.28-7.24(\mathrm{~m}, 1 \mathrm{H}, A r H), 7.19$ (t, $J=2.0 \mathrm{~Hz}, 1 \mathrm{H}, A r H), 7.08$ $(\mathrm{dd}, J=8.0,2.1 \mathrm{~Hz}, 1 \mathrm{H}, \mathrm{ArH}), 3.30\left(\mathrm{~s}, 3 \mathrm{H}, \mathrm{CH}_{3}\right)$.<smiles>O=CN(CC1CC2CCC1C2)c1ccc(Br)cc1</smiles>

$\boldsymbol{N}$-(4-bromophenyl)- $\boldsymbol{N}$-methylformamide (2i) (82\%) about 93:7 mixture of rotamers. Major rotamer: ${ }^{1} \mathbf{H} \mathbf{N M R}\left(500 \mathrm{MHz}, \mathrm{CDCl}_{3}\right) \delta=8.45(\mathrm{~s}, 1 \mathrm{H}, \mathrm{CHO}), 7.55-$ $7.50(\mathrm{~m}, 2 \mathrm{H}, \mathrm{ArH}), 7.05(\mathrm{~m}, 2 \mathrm{H}, \mathrm{ArH}), 3.30\left(\mathrm{~s}, 3 \mathrm{H}, \mathrm{CH}_{3}\right)$. 


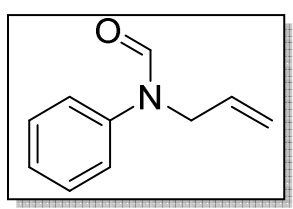

$\boldsymbol{N}$-allyl- $\boldsymbol{N}$-phenylformamide (2j) $\mathbf{( 7 0 \% )}$ about 93:7 mixture of rotamers. Major rotamer: ${ }^{1} \mathbf{H}$ NMR $\left(500 \mathrm{MHz}, \mathrm{CDCl}_{3}\right) \delta=8.50(\mathrm{~s}, 1 \mathrm{H}, \mathrm{CHO}), 7.40(\mathrm{dd}, J=11.0,4.7$ $\mathrm{Hz}, 2 \mathrm{H}, A r H), 7.32-7.25(\mathrm{~m}, 1 \mathrm{H}, A r H), 7.22-7.15(\mathrm{~m}, 2 \mathrm{H}, A r H), 5.93-5.79(\mathrm{~m}$, $1 \mathrm{H},=C H), 5.22-5.18\left(\mathrm{~m}, 1 \mathrm{H},=\mathrm{CH}_{2}\right), 5.18-5.14\left(\mathrm{~m}, 1 \mathrm{H},=\mathrm{CH}_{2}\right), 4.47-4.40(\mathrm{~m}$, $2 \mathrm{H}, \mathrm{NCH}_{2}$ ).<smiles>N#CCN(CO)c1ccccc1</smiles>

$\boldsymbol{N}$-(2-cyanoethyl)- $\boldsymbol{N}$-phenylformamide (2k) (32\%) about 93:7 mixture of rotamers. Major rotamer: ${ }^{1} \mathbf{H}$ NMR $\left(500 \mathrm{MHz}, \mathrm{CDCl}_{3}\right) \delta=8.38(\mathrm{~s}, 1 \mathrm{H}, \mathrm{CHO}), 7.47(\mathrm{dd}, J=$ 10.7, $4.8 \mathrm{~Hz}, 2 \mathrm{H}, A r H), 7.37$ (t, $J=7.4 \mathrm{~Hz}, 1 \mathrm{H}, A r H), 7.23$ (d, J=7.5 Hz, 2H, ArH), $4.07\left(\mathrm{t}, J=6.9 \mathrm{~Hz}, 2 \mathrm{H}, \mathrm{CH}_{2}\right), 2.70\left(\mathrm{t}, J=6.9 \mathrm{~Hz}, 2 \mathrm{H}, \mathrm{CH}_{2}\right)$.<smiles>O=CN(CCC1CC2CCCC1C2)c1ccccc1</smiles>

$\boldsymbol{N}$-ethyl- $\boldsymbol{N}$-phenylformamide (21) (71\%) about 93:7 mixture of rotamers. Major rotamer: ${ }^{1} \mathbf{H}$ NMR $\left(500 \mathrm{MHz}, \mathrm{CDCl}_{3}\right) \delta=8.36(\mathrm{~s}, 1 \mathrm{H}, \mathrm{CHO}), 7.42(\mathrm{dd}, J=11.0 \mathrm{~Hz}$, $4.7 \mathrm{~Hz}, 2 \mathrm{H}, A r H), 7.33-7.27(\mathrm{~m}, 1 \mathrm{H}, A r H), 7.17$ (d, $J=8.1 \mathrm{~Hz}, 2 \mathrm{H}, A r H), 3.87$ (q, $J$ $\left.=7.2 \mathrm{~Hz}, 2 \mathrm{H}, \mathrm{CH}_{2}\right), 1.16\left(\mathrm{t}, \mathrm{J}=7.2 \mathrm{~Hz}, 3 \mathrm{H}, \mathrm{CH}_{3}\right)$.<smiles>O=C1Cc2cccc3c2CCN3C1</smiles>

indoline-1-carbaldehyde (2m) $\mathbf{( 8 5 \% )}$ about 83:17 mixture of rotamers. ${ }^{\mathbf{1}} \mathbf{H}$ NMR $\left(500 \mathrm{MHz}, \mathrm{CDCl}_{3}\right) \delta=8.91(\mathrm{~s}, 0.7 \mathrm{H}, C H O), 8.49(\mathrm{~s}, 0.14 \mathrm{H}, C H O), 8.06(\mathrm{~d}, 0.14 \mathrm{H}$, CHO), $7.25-7.12(\mathrm{~m}, 3 \mathrm{H}, A r H), 7.04$ (td, $J=7.2,1.5 \mathrm{~Hz}, 1 \mathrm{H}, A r H), 4.09$ (t, $J=8.4$ $\left.\mathrm{Hz}, 0.29 \mathrm{H}, \mathrm{N}-\mathrm{CH}_{2}\right), 4.07-4.01\left(\mathrm{~m}, 1.51 \mathrm{H}, \mathrm{N}-\mathrm{CH}_{2}\right), 3.18\left(\mathrm{~d}, \mathrm{~J}=8.5 \mathrm{~Hz}, 0.2 \mathrm{H}, \mathrm{CH}_{2}\right)$, $3.16-3.11\left(\mathrm{~m}, 1.6 \mathrm{H}, \mathrm{CH}_{2}\right)$. 


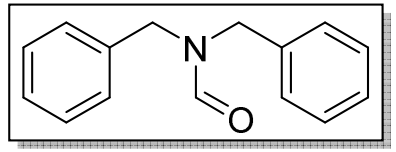

$\boldsymbol{N}, \boldsymbol{N}$-dibenzylformamide (2n) (86\%). ${ }^{1} \mathrm{H} \mathrm{NMR}\left(500 \mathrm{MHz}, \mathrm{CDCl}_{3}\right) \delta=8.45(\mathrm{~s}, 1 \mathrm{H}$, $C H O), 7.36(\mathrm{qd}, J=13.8,6.9 \mathrm{~Hz}, 6 \mathrm{H}, A r H), 7.21(\mathrm{dd}, J=12.8,7.1 \mathrm{~Hz}, 4 \mathrm{H}, A r H)$, $4.44\left(\mathrm{~s}, 2 \mathrm{H}, \mathrm{N}-\mathrm{CH}_{2}\right), 4.29\left(\mathrm{~s}, 2 \mathrm{H}, \mathrm{N}-\mathrm{CH}_{2}\right)$.

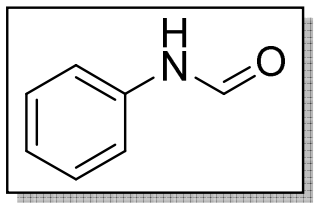

$\mathbf{N}$-phenylformamide (2p) (93\%) about 53:47 mixture of rotamers. ${ }^{\mathbf{1}} \mathbf{H}$ NMR (500 $\left.\mathrm{MHz}, \mathrm{CDCl}_{3}\right) \delta=8.92(\mathrm{br}, 0.52 \mathrm{H}, N H), 8.70(\mathrm{~d}, J=11.4 \mathrm{~Hz}, 0.53 \mathrm{H}, C H O), 8.35(\mathrm{~d}, J$ $=1.8 \mathrm{~Hz}, 0.47 \mathrm{H}, C H O), 8.06(\mathrm{br}, 0.45 \mathrm{H}, N H), 7.55(\mathrm{~d}, J=7.6 \mathrm{~Hz}, 1 \mathrm{H}, A r H), 7.40-$ $7.28(\mathrm{~m}, 2 \mathrm{H}, A r H), 7.21-7.08(\mathrm{~m}, 2 \mathrm{H}, \mathrm{ArH})$.<smiles>COc1ccc(NC=O)cc1</smiles>

$\mathbf{N}$-(4-methoxyphenyl)formamide (2q) $\mathbf{( 9 0 \% )}$ about 55:45 mixture of rotamers. ${ }^{\mathbf{1}} \mathbf{H}$ NMR $\left(500 \mathrm{MHz}, \mathrm{CDCl}_{3}\right) \delta=8.50(\mathrm{~d}, J=11.0 \mathrm{~Hz}, 1 \mathrm{H}, \mathrm{CHO}$ and $\mathrm{NH}$ overlap $), 8.28$ $(\mathrm{d}, J=1.8 \mathrm{~Hz}, 0.55 \mathrm{H}, C H O), 7.82(\mathrm{~s}, 0.53 \mathrm{H}, N H), 7.46-7.44(\mathrm{~m}, 0.51 \mathrm{H}, A r H), 7.44$ - $7.41(\mathrm{~m}, 0.55 \mathrm{H}, A r H), 7.05-7.03(\mathrm{~m}, 0.45 \mathrm{H}, A r H), 7.03-7.01(\mathrm{~m}, 0.54 \mathrm{H}, A r H)$, $6.89-6.87(\mathrm{~m}, 0.53 \mathrm{H}, A r H), 6.87-6.85(\mathrm{~m}, 0.46 \mathrm{H}, A r H), 6.85-6.84(\mathrm{~m}, 0.52 \mathrm{H}$, $\mathrm{ArH}), 6.84-6.82(\mathrm{~m}, 0.51 \mathrm{H}, \mathrm{ArH}), 3.79\left(\mathrm{~s}, 1.49 \mathrm{H}, \mathrm{OCH}_{3}\right), 3.77\left(\mathrm{~s}, 1.60 \mathrm{H}, \mathrm{OCH}_{3}\right)$.<smiles>O=CNc1ccc(CBr)cc1</smiles>

$\mathbf{N}$-(4-butylphenyl)formamide (2r) (70\%) about 55:45 mixture of rotamers. ${ }^{\mathbf{1}} \mathbf{H}$ NMR $\left(500 \mathrm{MHz}, \mathrm{CDCl}_{3}\right) \delta=8.82(\mathrm{~s}, 0.48 \mathrm{H}, N H), 8.64(\mathrm{~d}, J=11.4 \mathrm{~Hz}, 0.5 \mathrm{H}, \mathrm{CHO}), 8.32$ (s, 0.42H,CHO), 7.95 (s, 0.42H, NH), $7.45(\mathrm{~d}, J=8.3 \mathrm{~Hz}, 0.95 \mathrm{H}, A r H), 7.14$ (dd, $J=$ 15.3, 8.2 Hz, 2.05H, $A r H), 7.01$ (d, $J=8.3 \mathrm{~Hz}, 1.10 \mathrm{H}, A r H), 2.58$ (dt, $J=10.1,7.8 \mathrm{~Hz}$, $\left.1.91 \mathrm{H}, \mathrm{CH}_{2}\right), 1.57\left(\mathrm{dd}, J=14.8,7.9 \mathrm{~Hz}, 1.91 \mathrm{H}, \mathrm{CH}_{2}\right), 1.34(\mathrm{dt}, J=13.2,7.3 \mathrm{~Hz}$, $\left.1.93 \mathrm{H}, \mathrm{CH}_{2}\right), 0.92\left(\mathrm{dd}, \mathrm{J}=13.3,7.3 \mathrm{~Hz}, 2.84 \mathrm{H}, \mathrm{CH}_{3}\right)$. 


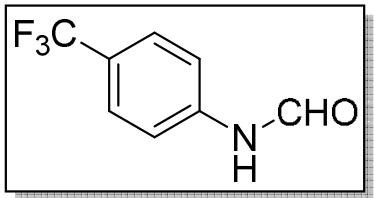

$\mathrm{N}$-(4-(trifluoromethyl)phenyl)formamide (2s) (42\%) about 50:50 mixture of rotamers. ${ }^{1} \mathbf{H}$ NMR $\left(500 \mathrm{MHz}, \mathrm{CDCl}_{3}\right) \delta=8.80(\mathrm{~d}, J=10.6 \mathrm{~Hz}, 1 \mathrm{H}, \mathrm{CHO}$ and $\mathrm{NH}$ overlap), $8.41(\mathrm{~d}, J=1.4 \mathrm{~Hz}, 0.71 \mathrm{H}, C H O), 7.78(\mathrm{br}, 0.6 \mathrm{H}, N H), 7.67(\mathrm{~d}, J=8.5 \mathrm{~Hz}$, 1.47H, $A r H), 7.61(\mathrm{~d}, J=8.4 \mathrm{~Hz}, 1.03 \mathrm{H}, A r H), 7.58(\mathrm{~d}, J=8.6 \mathrm{~Hz}, 1.55 \mathrm{H}, A r H), 7.19$ (d, $J=8.4 \mathrm{~Hz}, 1.19 \mathrm{H}, A r H)$.<smiles>O=CNCCNc1ccccc1</smiles>

$\boldsymbol{N}$-(2-(phenylamino)ethyl)formamide (2u) (65\%) about 86:14 mixture of rotamers. ${ }^{1}$ H NMR $\left(500 \mathrm{MHz}, \mathrm{CDCl}_{3}\right) \delta=8.17(\mathrm{~s}, 0.86 \mathrm{H}, \mathrm{CHO}), 7.98(\mathrm{~d}, J=11.9 \mathrm{~Hz}, 0.14 \mathrm{H}$, $C H O), 7.21(\mathrm{dd}, J=10.6,5.2 \mathrm{~Hz}, 2 \mathrm{H}, A r H), 6.76(\mathrm{q}, J=7.4 \mathrm{~Hz}, 1 \mathrm{H}, A r H), 6.63(\mathrm{t}, J$ $=8.4 \mathrm{~Hz}, 2 \mathrm{H}, A r H), 6.36(\mathrm{br}, 1 \mathrm{H}, N H C O), 4.04(\mathrm{~s}, 1 \mathrm{H}, N H A r), 3.53(\mathrm{dd}, J=11.8,5.9$ $\left.\mathrm{Hz}, 1.95 \mathrm{H}, \mathrm{CH}_{2} \mathrm{NHCHO}\right), 3.37\left(\mathrm{dd}, \mathrm{J}=11.9,5.9 \mathrm{~Hz}, 0.35 \mathrm{H}, \mathrm{CH}_{2} \mathrm{NHCHO}\right), 3.30(\mathrm{t}, \mathrm{J}$ $\left.=5.8 \mathrm{~Hz}, 1.86 \mathrm{H}, \mathrm{CH}_{2} \mathrm{NHAr}\right), 3.28-3.24\left(\mathrm{~m}, 0.3 \mathrm{H}, \mathrm{CH}_{2} \mathrm{NHAr}\right)$.<smiles>O=C(NN=C(P)Pc1ccccc1)c1ccccc1</smiles>

$N^{\prime}$-(diphenylmethylene)formohydrazide (2v) (60\%). ${ }^{\mathbf{1}} \mathbf{H} \mathbf{N M R}\left(500 \mathrm{MHz}, \mathrm{CDCl}_{3}\right)$ $\delta=8.84(\mathrm{dd}, J=10.5,1.0 \mathrm{~Hz}, 1 \mathrm{H}, C H O), 8.55(\mathrm{~m}, 1 \mathrm{H}, N H), 7.52(\mathrm{~m}, 5 \mathrm{H}, A r H), 7.43$ $-7.30(\mathrm{~m}, 3 \mathrm{H}, \operatorname{ArH}), 7.27-7.18(\mathrm{~m}, 2 \mathrm{H}, \operatorname{ArH})$.

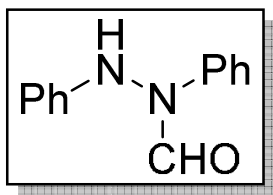

$\boldsymbol{N}, \boldsymbol{N}^{\prime}$-diphenylformohydrazide (2w) $\mathbf{( 4 2 \% )}$ ) about 73:27 mixture of rotamers. ${ }^{\mathbf{1}} \mathbf{H}$ NMR $\left(500 \mathrm{MHz}, \mathrm{CDCl}_{3}\right) \delta=8.88(\mathrm{~s}, 0.73 \mathrm{H}, \mathrm{CHO}), 8.62(\mathrm{~s}, 0.27 \mathrm{H}, \mathrm{CHO}), 7.68(\mathrm{~d}, J$ $=8.0 \mathrm{~Hz}, 0.67 \mathrm{H}, A r H), 7.54-7.42(\mathrm{~m}, 3.04 \mathrm{H}, A r H), 7.36(\mathrm{t}, J=8.0 \mathrm{~Hz}, 0.7 \mathrm{H}, A r H)$, $7.29(\mathrm{~m}, 1 \mathrm{H}, A r H), 7.25$ (t, $J=8.0 \mathrm{~Hz}, 1.91 \mathrm{H}, A r H), 7.19$ (t, J=7.4Hz, 0.28H, $A r H)$, $6.99-6.90(\mathrm{~m}, 1.76 \mathrm{H}, A r H$ and $N H), 6.84$ (t, $J=9.0 \mathrm{~Hz}, 1.94 \mathrm{H}, A r H), 6.68$ (s, 0.24H, $\mathrm{NH})$. 


\section{$\mathrm{Ph}_{\mathrm{N}^{-}} \mathrm{Ph}$ \\ HN \\ $\mathrm{CHO}$}

$\boldsymbol{N}^{\prime}, \boldsymbol{N}^{\prime}$-diphenylformohydrazide (2x) $\mathbf{( 3 5 \% )}$ about $70: 30$ mixture of rotamers. ${ }^{\mathbf{1}} \mathbf{H}$ NMR $\left(500 \mathrm{MHz}, \mathrm{CDCl}_{3}\right) \delta=8.42(\mathrm{~d}, J=11.1 \mathrm{~Hz}, 0.7 \mathrm{H}, \mathrm{CHO}), 8.19(\mathrm{~s}, 1 \mathrm{H}, 0.3 \mathrm{H}$, CHO), $7.88(\mathrm{~m}, 1 \mathrm{H}, N H), 7.33(\mathrm{dd}, J=11.3,4.6 \mathrm{~Hz}, 3.46 \mathrm{H}), 7.29(\mathrm{~m}, 1.69 \mathrm{H}), 7.11(\mathrm{~m}$, $6.08 \mathrm{H}), 7.05(\mathrm{t}, J=7.4 \mathrm{~Hz}, 0.89 \mathrm{H})$.
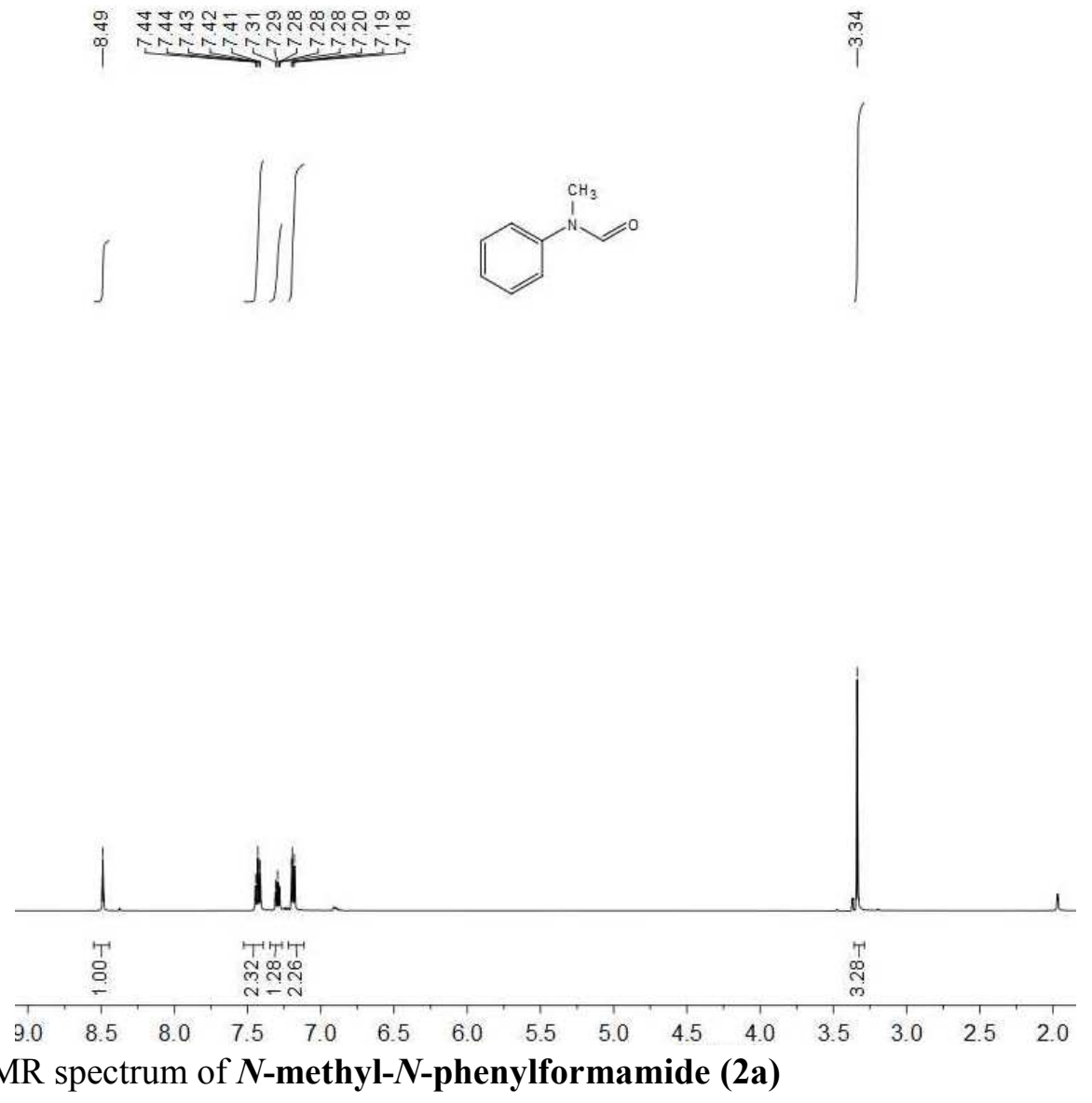


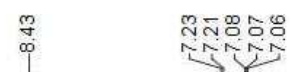

i nisin
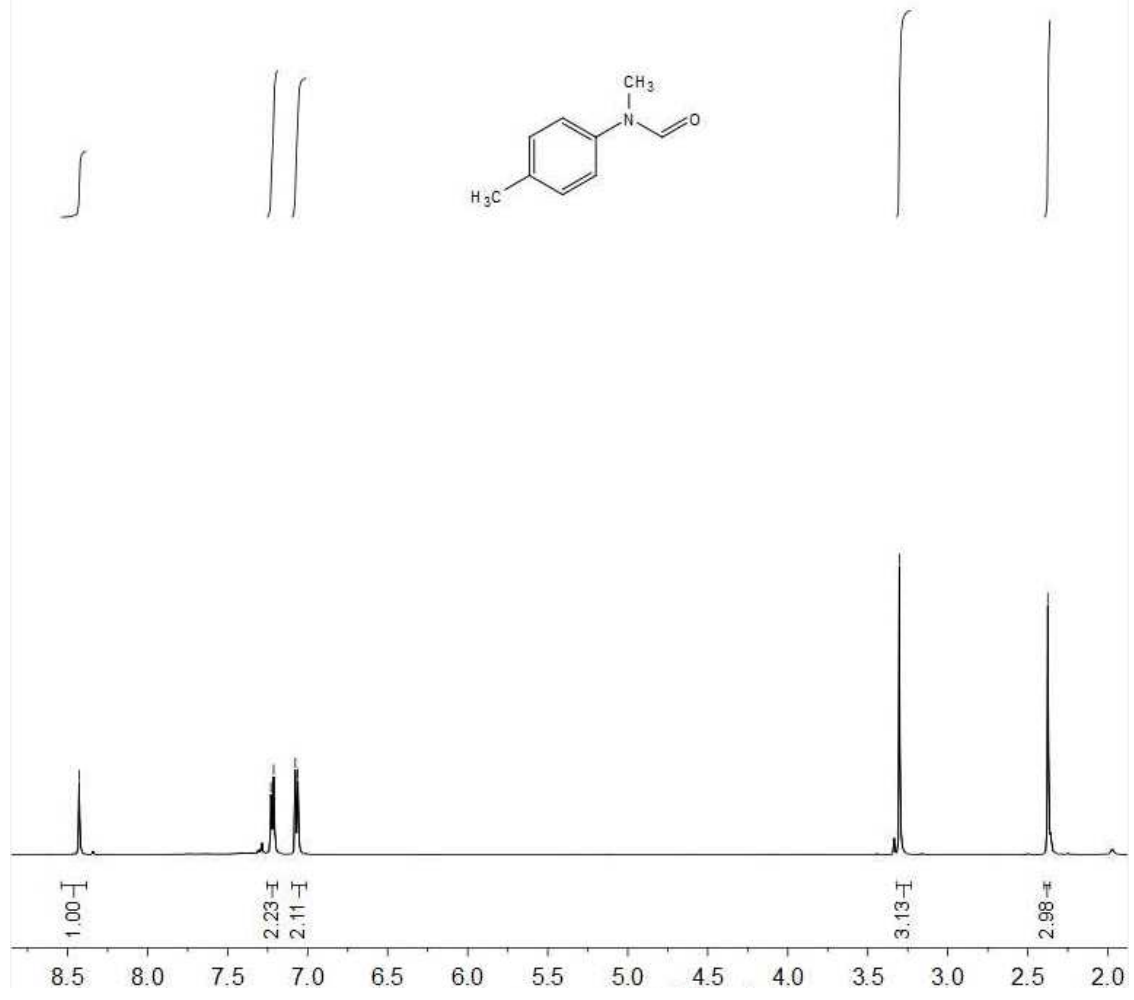

${ }^{1} \mathrm{H}$ NMR spectrum of $\boldsymbol{N}$-methyl- $\boldsymbol{N}$-(p-tolyl)formamide (2g) 

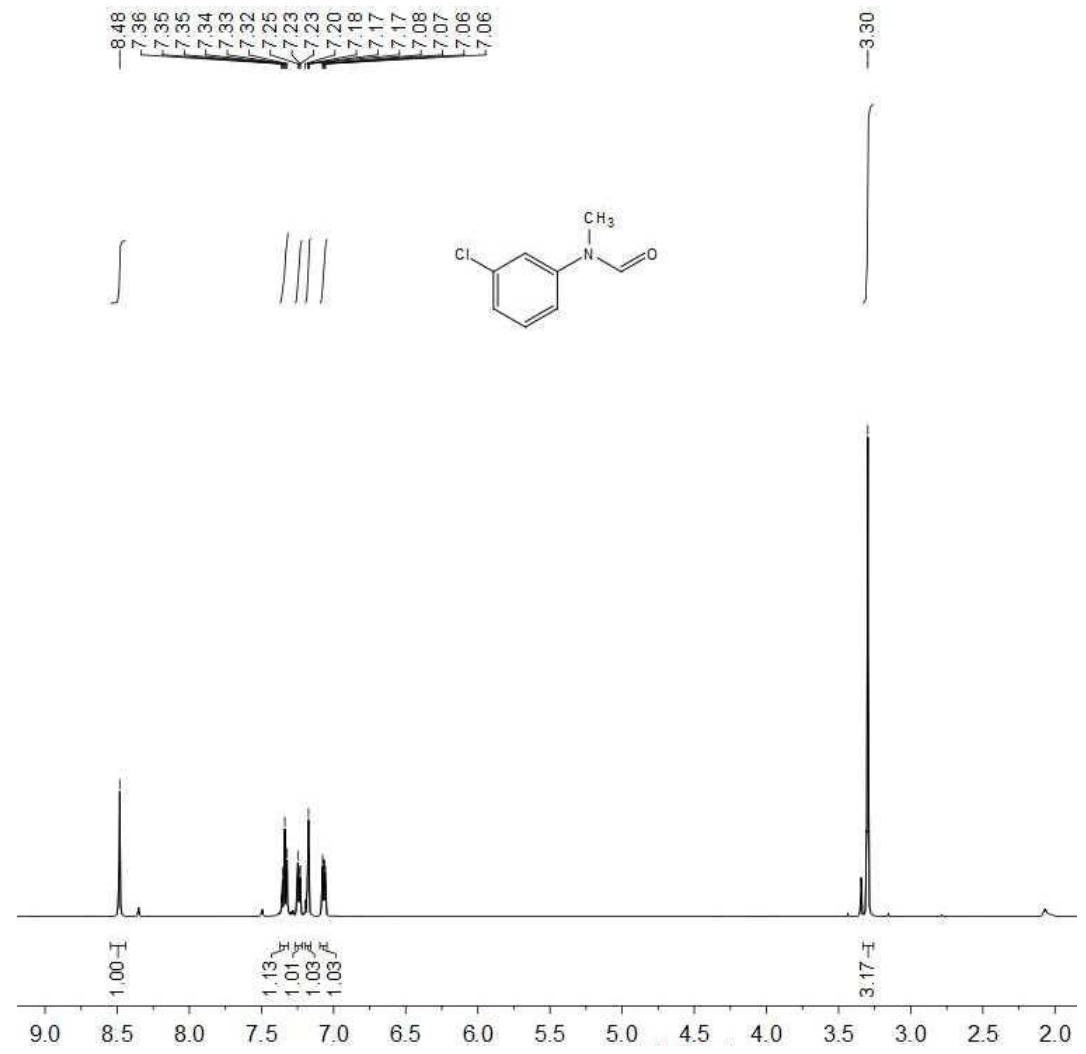

${ }^{1} \mathrm{H}$ NMR spectrum of $\mathrm{N}$-(3-chlorophenyl)- $\mathrm{N}$-methylformamide (2h) 


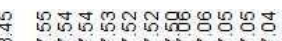

罂

$\stackrel{0}{p}$
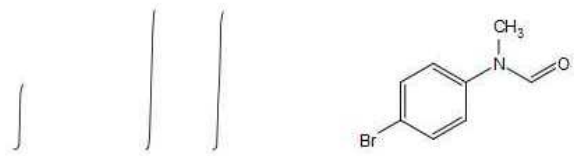

\section{(n)}

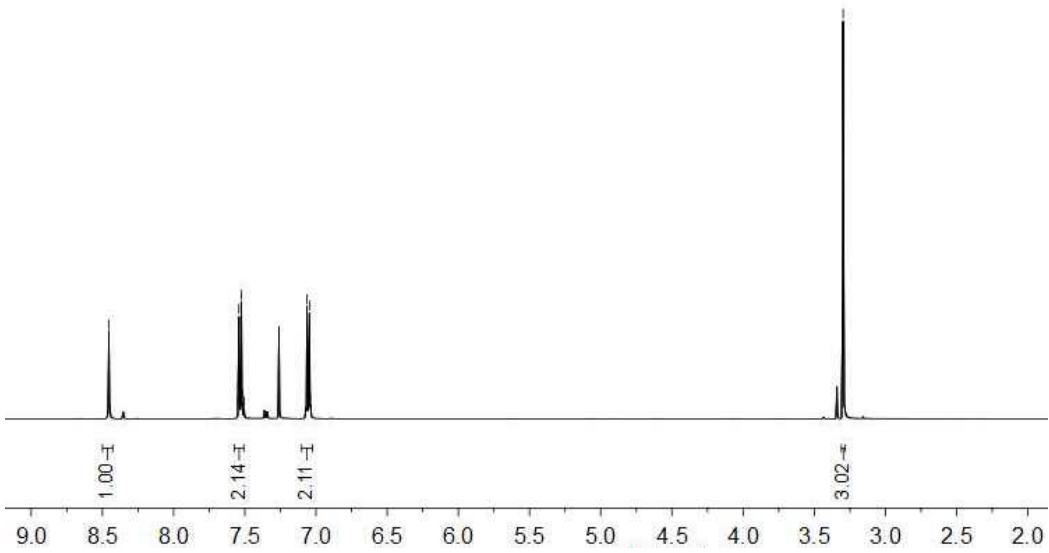

${ }^{1} \mathrm{H}$ NMR spectrum of $\boldsymbol{N}$-(4-bromophenyl)- $\boldsymbol{N}$-methylformamide (2i) 


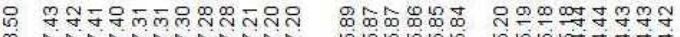

क N
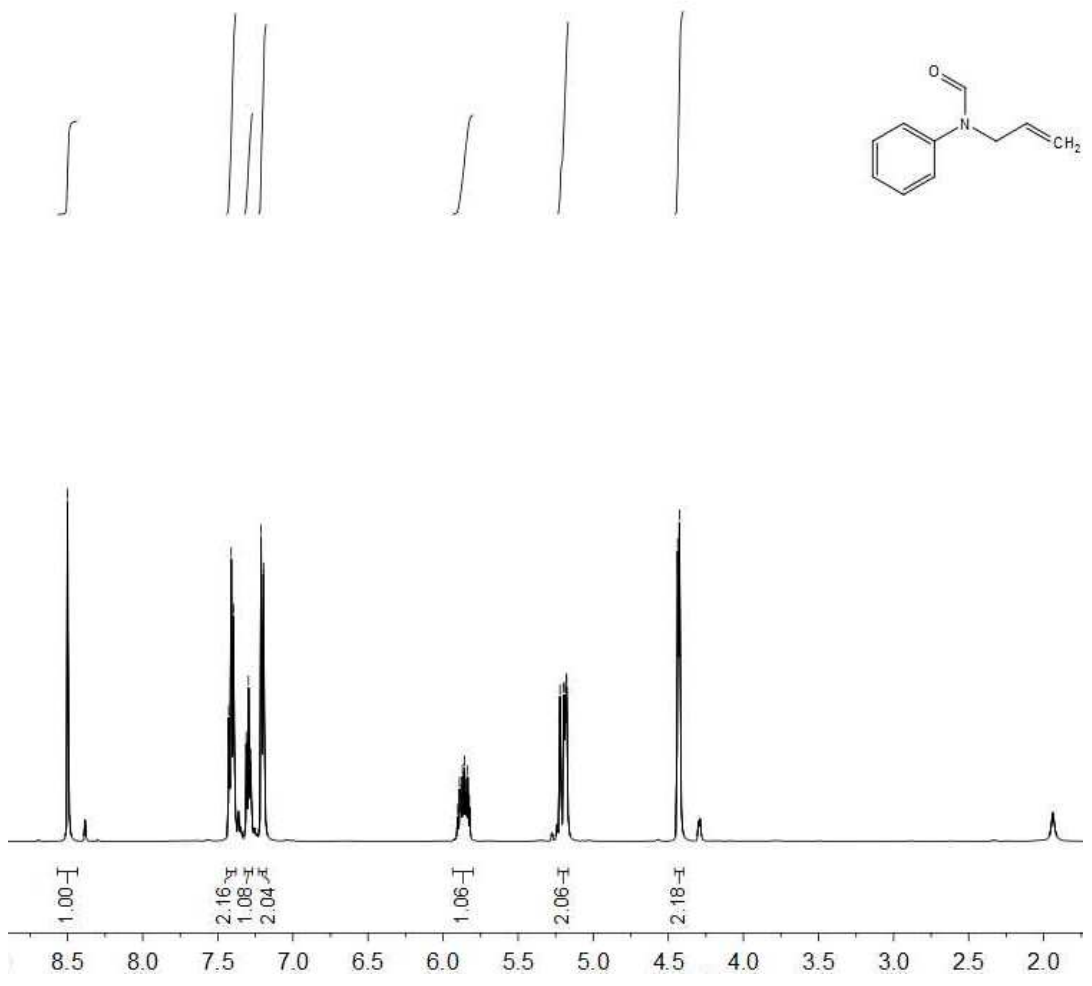

${ }^{1} \mathrm{H}$ NMR spectrum of $\boldsymbol{N}$-allyl- $\boldsymbol{N}$-phenylformamide (2j) 


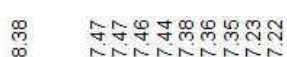

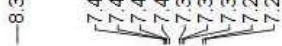

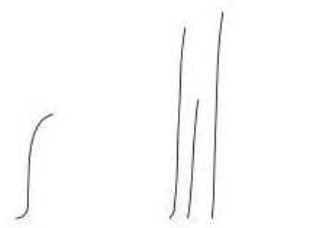

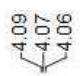

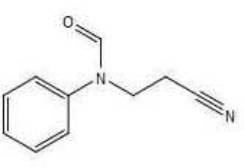

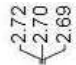

$\mid$

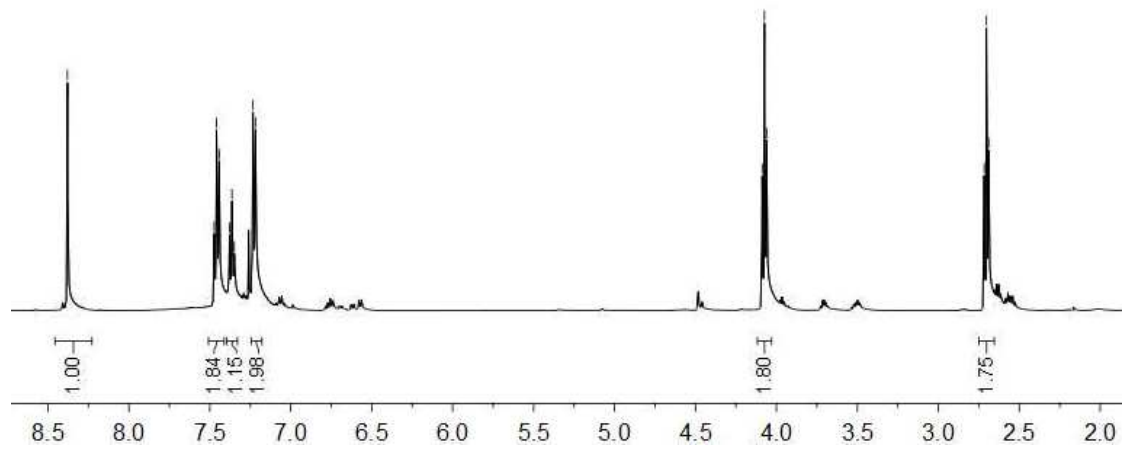

${ }^{1} \mathrm{H}$ NMR spectrum of $\boldsymbol{N}$-(2-cyanoethyl)- $\boldsymbol{N}$-phenylformamide (2k) 


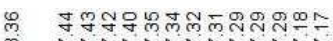

i) inivinasin

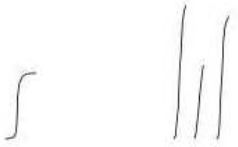

CO $\stackrel{\infty}{=}=\frac{10}{2}$

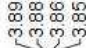

(

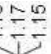

1

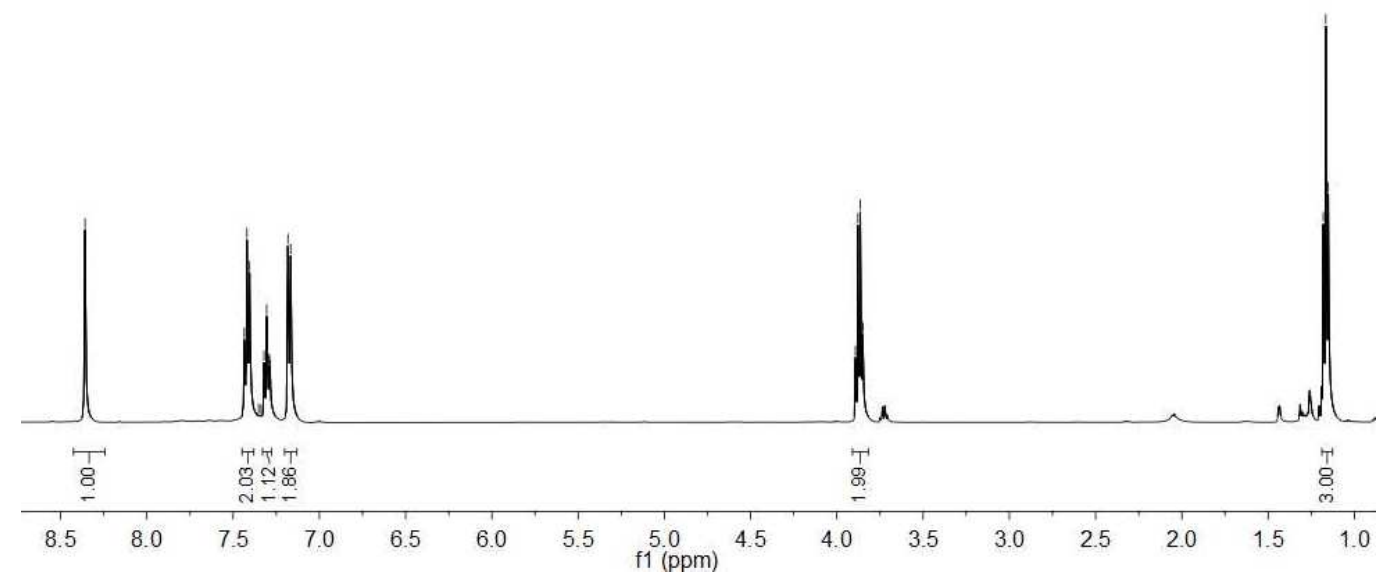

${ }^{1} \mathrm{H}$ NMR spectrum of $\boldsymbol{N}$-ethyl- $\boldsymbol{N}$-phenylformamide (2I) 

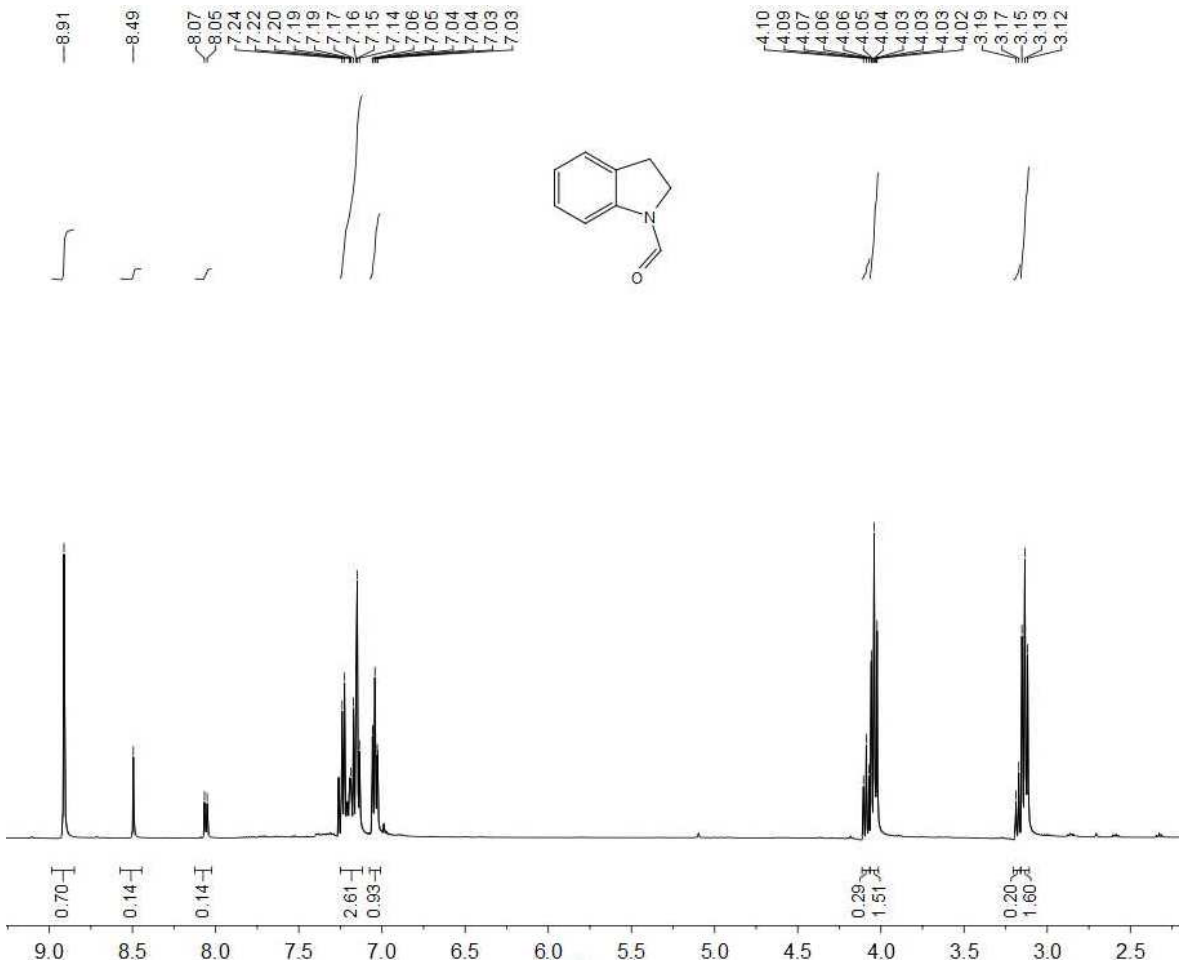

${ }^{1}$ H NMR spectrum of indoline-1-carbaldehyde (2m) 

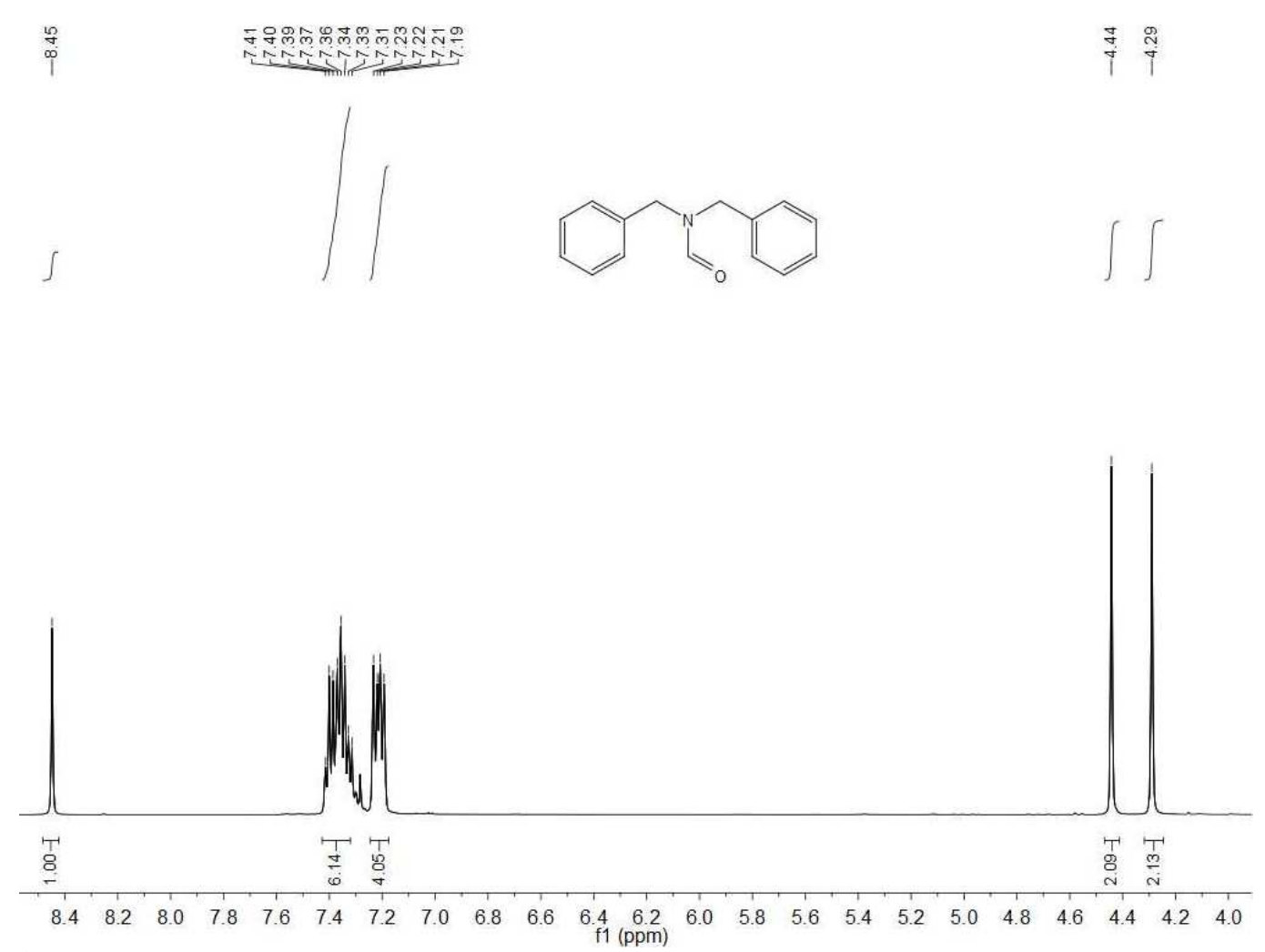

${ }^{1} \mathrm{H}$ NMR spectrum of $\boldsymbol{N}, \boldsymbol{N}$-dibenzylformamide (2n) 


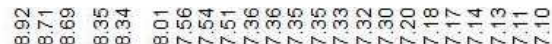

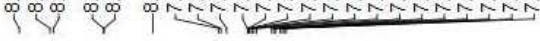

$\iiint \int d \mid$<smiles>O=CNc1ccccc1</smiles>

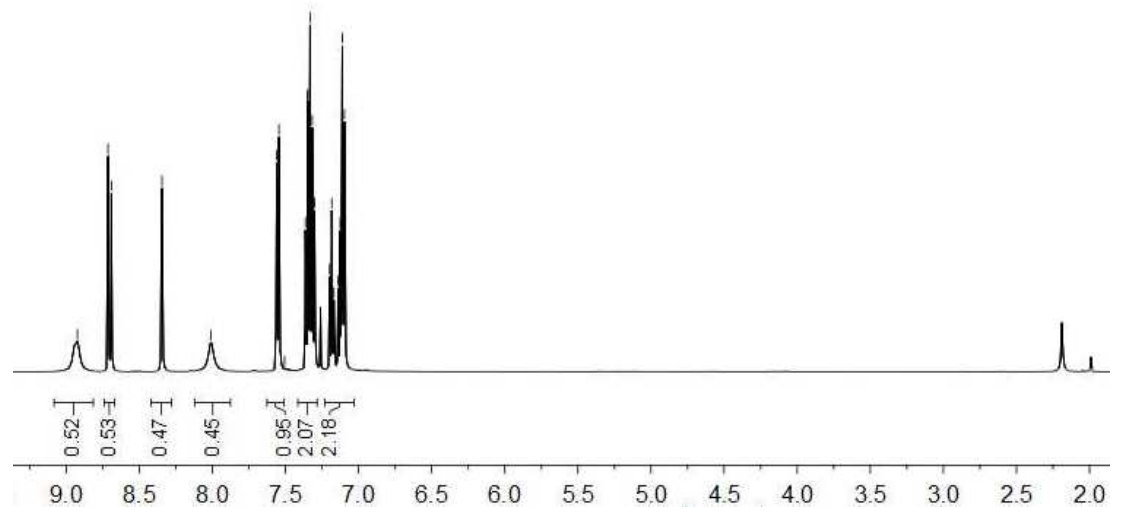

${ }^{1} \mathrm{H}$ NMR spectrum of $\boldsymbol{N}$-phenylformamide (2p) 

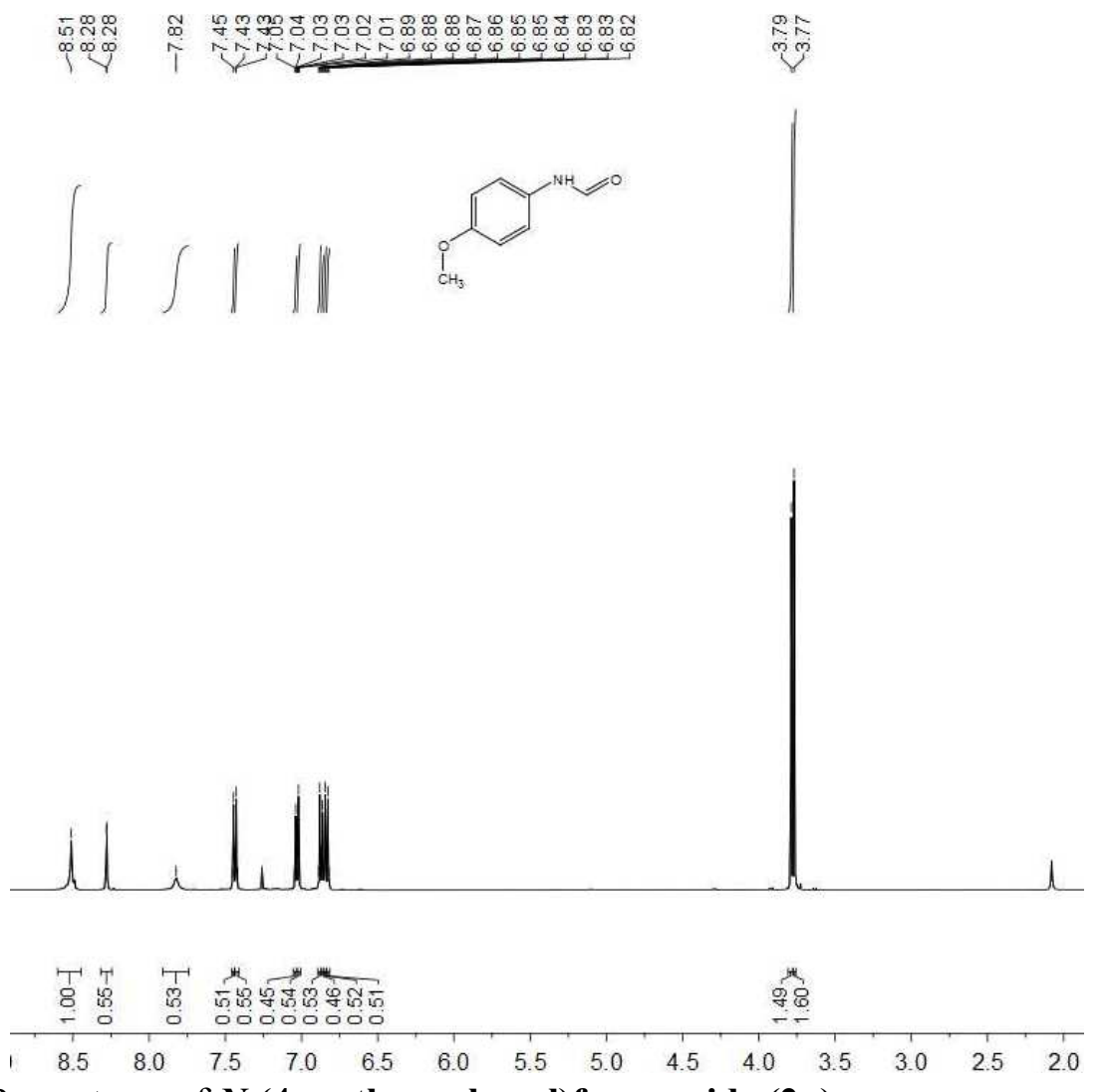

${ }^{1} \mathrm{H}$ NMR spectrum of $\boldsymbol{N}$-(4-methoxyphenyl)formamide (2q) 


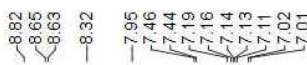

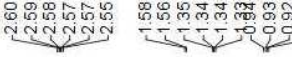
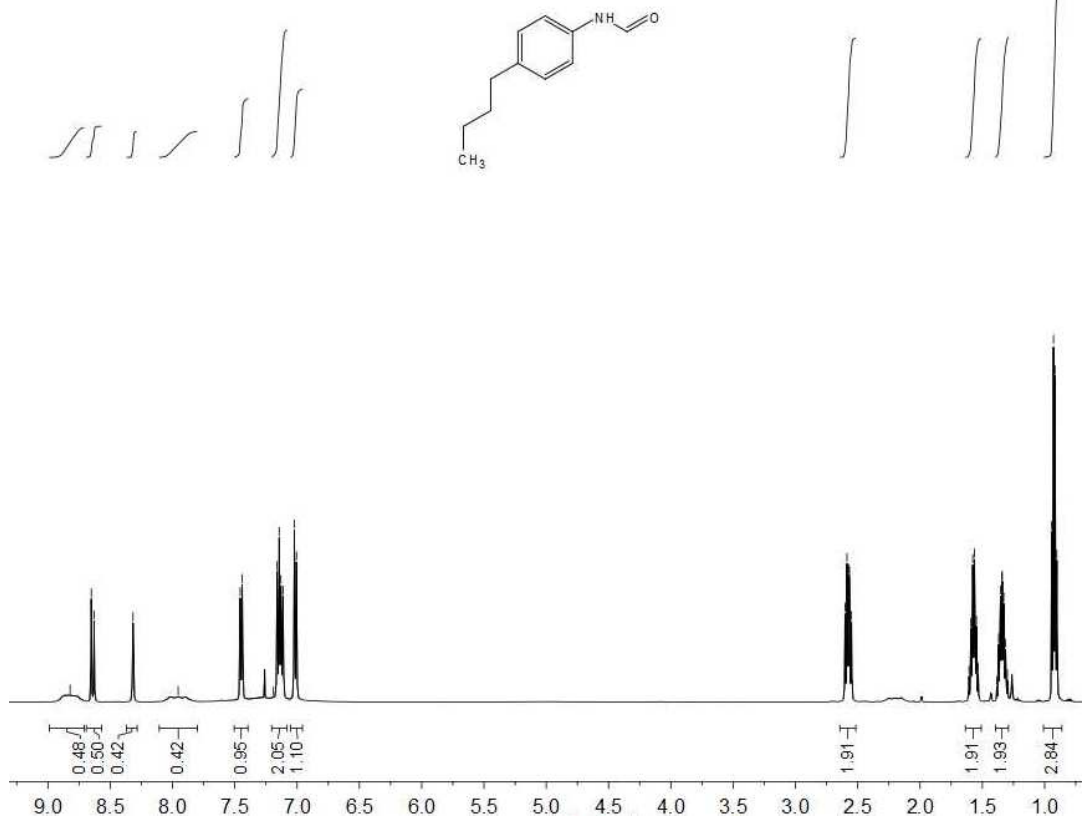

${ }^{1} \mathrm{H}$ NMR spectrum of $\boldsymbol{N}$-(4-butylphenyl)formamide (2r) 

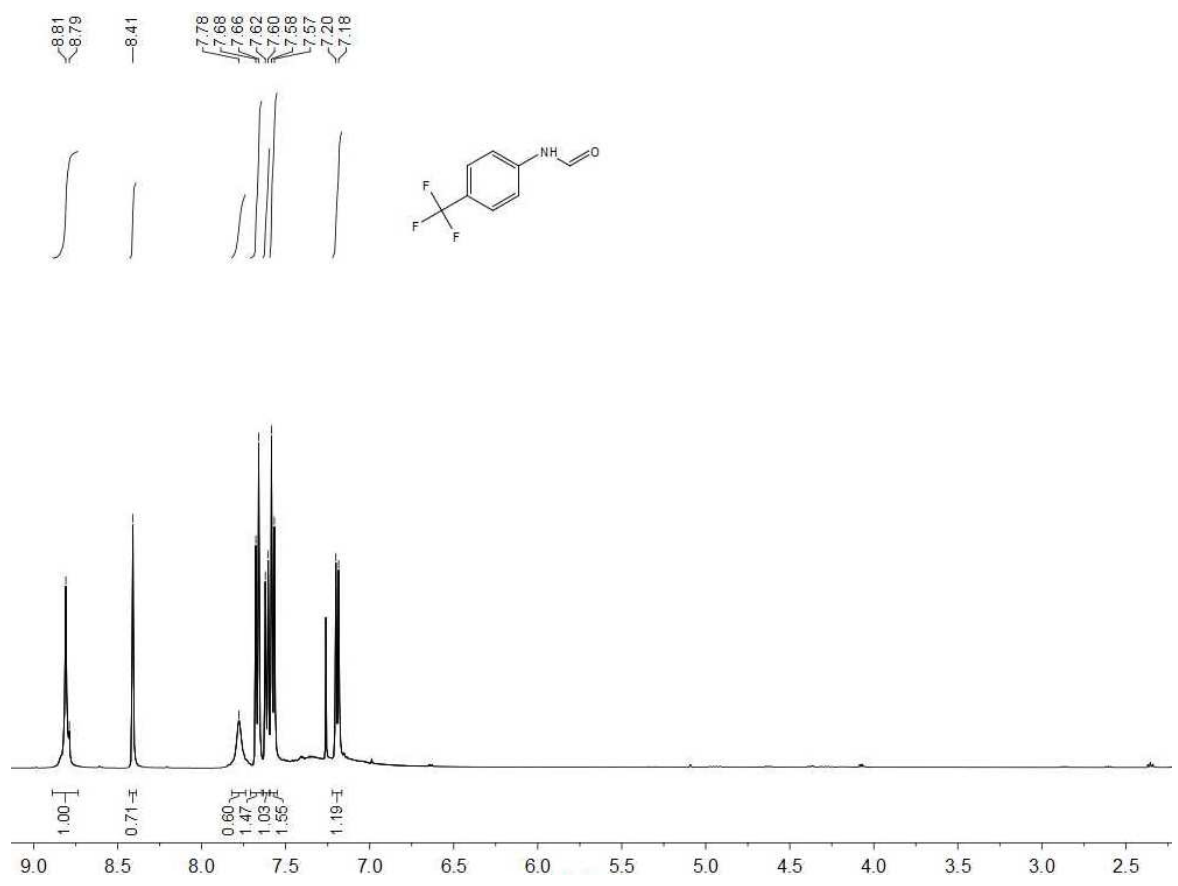

${ }^{1} \mathrm{H}$ NMR spectrum of $\mathrm{N}$-(4-(trifluoromethyl)phenyl)formamide (2s) 

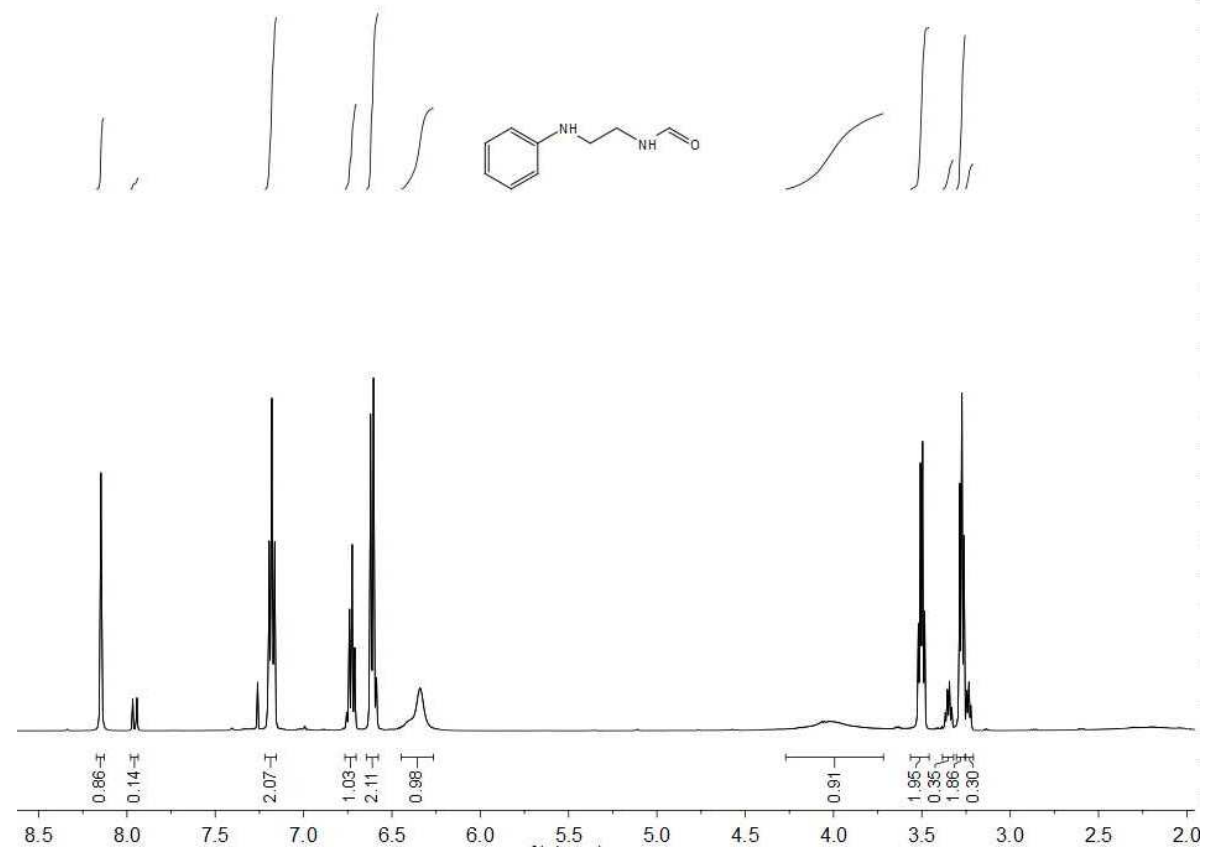

${ }^{1} \mathrm{H}$ NMR spectrum of $\mathbf{N}$-(2-(phenylamino)ethyl)formamide (2u) 


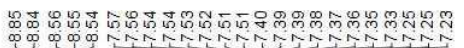

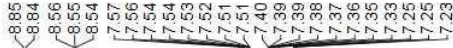
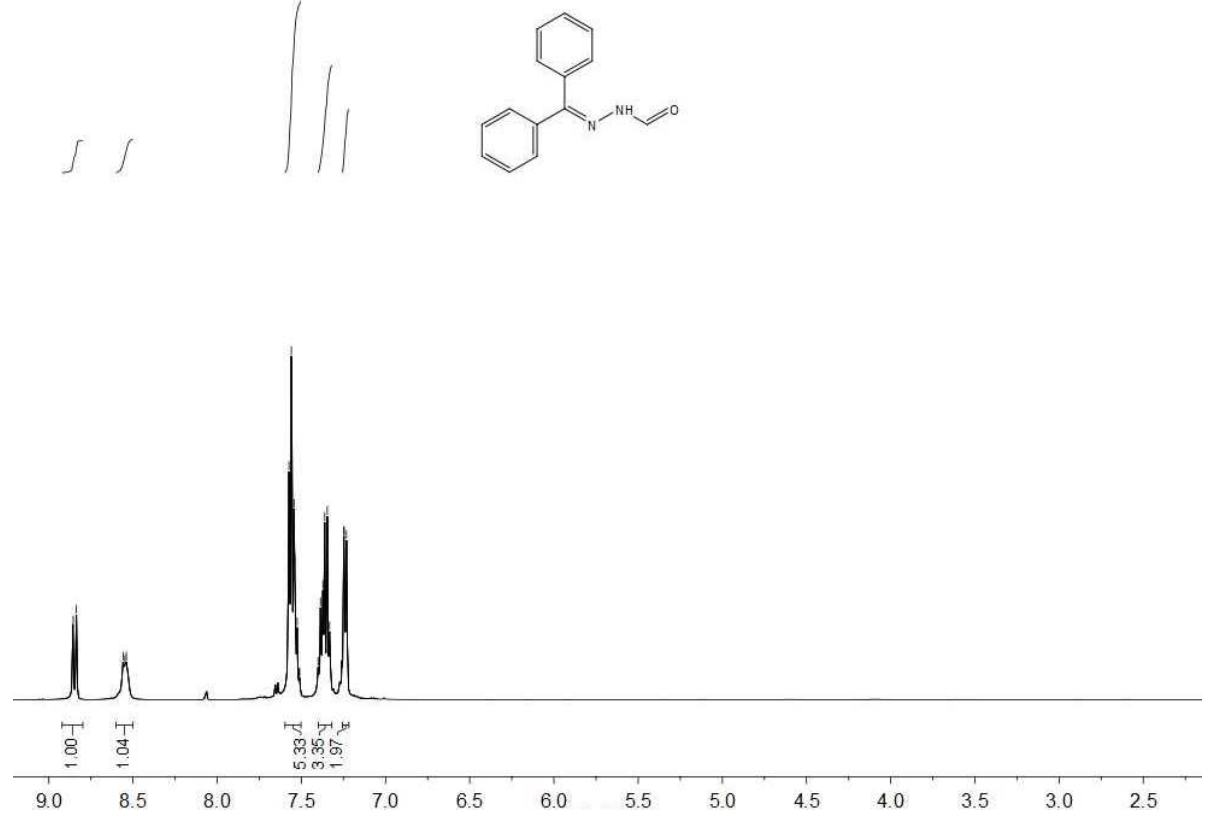

${ }^{1} \mathrm{H}$ NMR spectrum of $N^{\prime}$-(diphenylmethylene)formohydrazide (2v) 


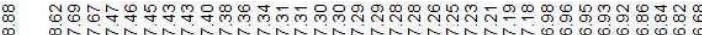

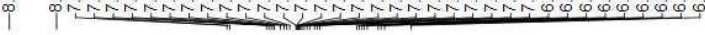

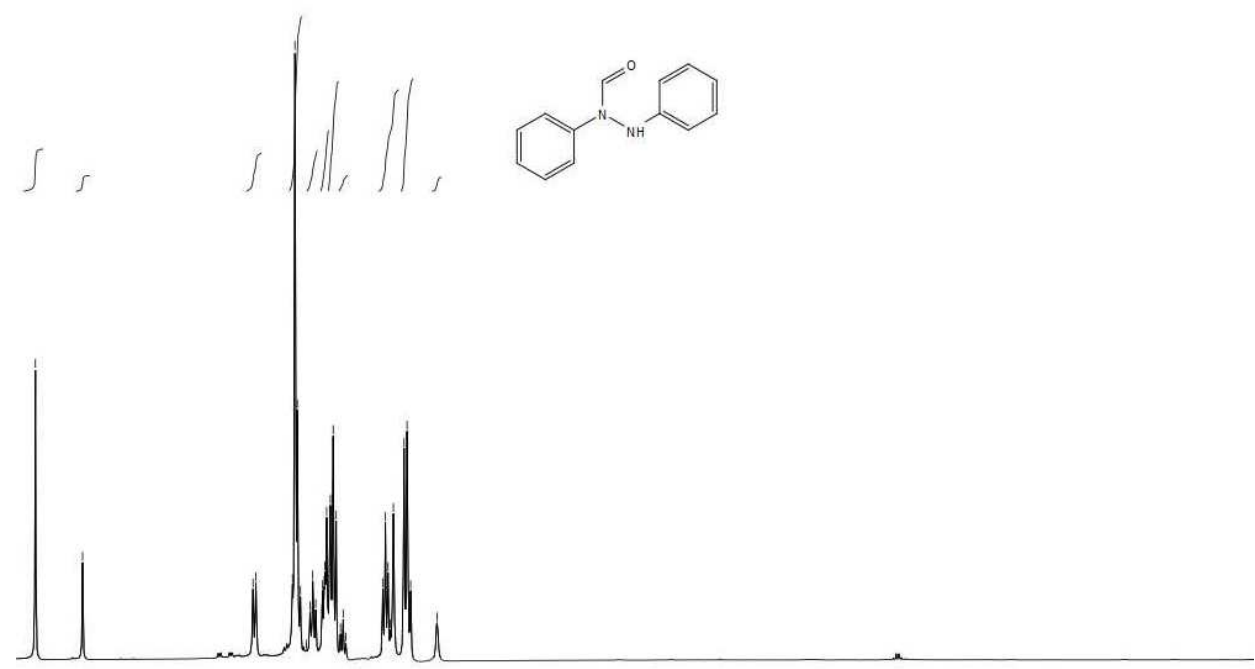

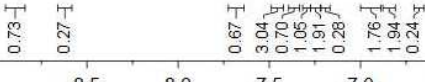

$\begin{array}{lll}8.5 & 80 & 75\end{array}$

${ }^{1} \mathrm{H}$ NMR spectrum of $\boldsymbol{N}, \boldsymbol{N}^{\prime}$-diphenylformohydrazide (2w) 


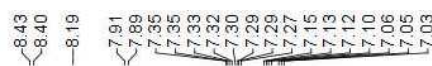

$1,1||_{1}$

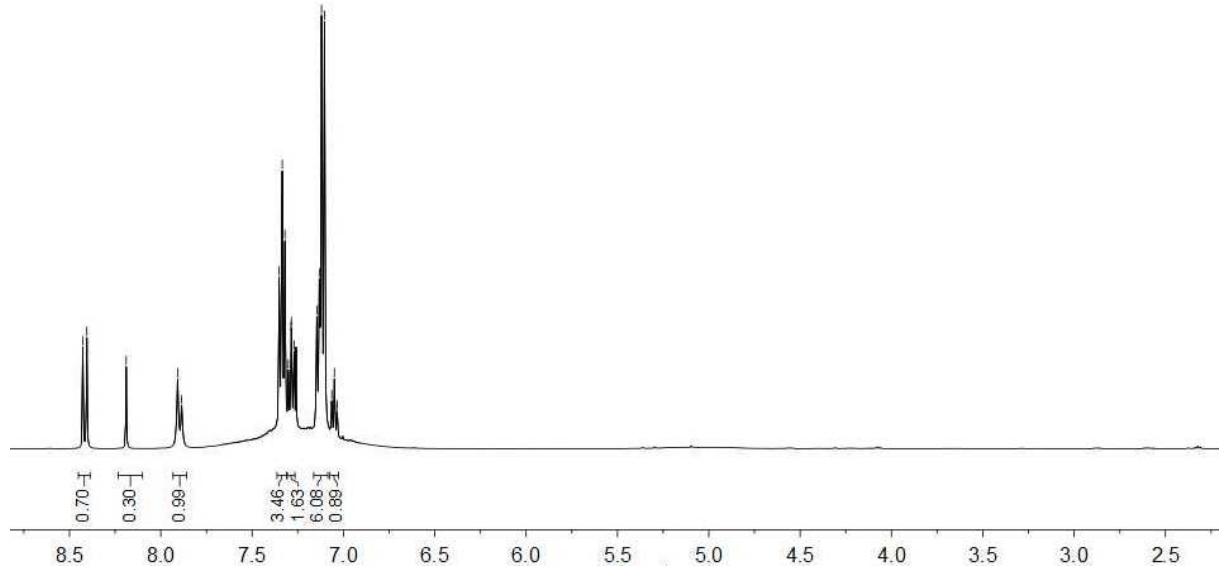

${ }^{1} \mathrm{H}$ NMR spectrum of $\boldsymbol{N}^{\prime}, \boldsymbol{N}^{\prime}$-diphenylformohydrazide (2x) 


\section{Characterization of the methylated products}

Methylated products were identified through comparisons with the corresponding ${ }^{1} \mathrm{H}$ NMR data reported in the literatures.

$\mathbf{3 a}{ }^{16}, \mathbf{3} \mathbf{b}^{17}, \mathbf{3} \mathbf{c}^{17}, \mathbf{3} \mathbf{d}^{16}, \mathbf{3} \mathbf{e}^{18}, \mathbf{3} \mathbf{f}^{17}, \mathbf{3} \mathbf{g}^{18}, \mathbf{3} \mathbf{h}^{19}, \mathbf{3 i}^{18}, \mathbf{3 j}^{18}, \mathbf{3} \mathbf{k}^{18}, \mathbf{3} \mathbf{l}^{18}, \mathbf{3} \mathbf{m}^{18}, \mathbf{3} \mathbf{n}^{3}, \mathbf{3} \mathbf{o}^{17}, \mathbf{3} \mathbf{p}^{20}$, $\mathbf{3} \mathbf{q}^{18}, \mathbf{3} \mathbf{r}^{16}, \mathbf{3} \mathbf{s}^{21}, \mathbf{3 t ^ { 2 2 }}$.

${ }^{1} \mathrm{H}$ NMR data for the products with isolated yields in Table 5<smiles>c1ccc(N2CC3CCC2C3)cc1</smiles>

$\boldsymbol{N}, \mathbf{N}$,4-trimethylaniline (3b) (82\%). ${ }^{\mathbf{1}} \mathbf{H}$ NMR $\left(500 \mathrm{MHz}, \mathrm{CDCl}_{3}\right) \delta=7.06(\mathrm{~d}, J=8.4$ $\mathrm{Hz}, 2 \mathrm{H}), 6.70(\mathrm{~d}, J=8.8 \mathrm{~Hz}, 2 \mathrm{H}), 2.90(\mathrm{~s}, 6 \mathrm{H}), 2.26(\mathrm{~s}, 3 \mathrm{H})$.<smiles>Brc1ccc(N2CC3CCC2C3)cc1</smiles>

4-bromo- $\boldsymbol{N}$, $\boldsymbol{N}$-dimethylaniline (3d) (85\%). ${ }^{\mathbf{1}} \mathbf{H}$ NMR $\left(500 \mathrm{MHz}, \mathrm{CDCl}_{3}\right) \delta=7.30(\mathrm{~d}$, $J=9.2 \mathrm{~Hz}, 2 \mathrm{H}), 6.59(\mathrm{~d}, J=7.2 \mathrm{~Hz}, 1 \mathrm{H}), 2.93(\mathrm{~s}, 6 \mathrm{H})$.<smiles>Clc1ccc(N2CC3CCC2C3)cc1</smiles>

3-chloro- $\boldsymbol{N}, \boldsymbol{N}$-dimethylaniline (3e) (76\%). ${ }^{\mathbf{1}} \mathbf{H} \mathbf{N M R}\left(500 \mathrm{MHz}, \mathrm{CDCl}_{3}\right) \delta=7.18(\mathrm{t}$, $J=8.3 \mathrm{~Hz}, 1 \mathrm{H}), 6.72(\mathrm{~d}, J=6.8 \mathrm{~Hz}, 2 \mathrm{H}), 6.64(\mathrm{~d}, J=7.8 \mathrm{~Hz}, 1 \mathrm{H}), 2.98(\mathrm{~s}, 6 \mathrm{H})$.<smiles>c1ccc(N2CC3CCC2CC3)cc1</smiles>

$\boldsymbol{N}$-ethyl- $\boldsymbol{N}$-methylaniline (3g) (90\%). ${ }^{\mathbf{1}} \mathbf{H}$ NMR $\left(500 \mathrm{MHz}, \mathrm{CDCl}_{3}\right) \delta=7.23-7.21$ $(\mathrm{m}, 2 \mathrm{H}), 6.73-6.67(\mathrm{~m}, 3 \mathrm{H}), 3.40(\mathrm{q}, J=7.1 \mathrm{~Hz}, 2 \mathrm{H}), 2.90(\mathrm{~s}, 3 \mathrm{H}), 1.11(\mathrm{t}, J=7.1$ $\mathrm{Hz}, 3 \mathrm{H})$.

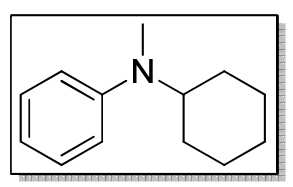


$\boldsymbol{N}$-cyclohexyl- $\boldsymbol{N}$-methylaniline (3h) (88\%). ${ }^{\mathbf{1}} \mathbf{H}$ NMR $\left(500 \mathrm{MHz}, \mathrm{CDCl}_{3}\right) \delta=7.22$ $(\mathrm{dd}, J=8.6,8.6 \mathrm{~Hz}, 2 \mathrm{H}), 6.77$ (d, $J=8.6 \mathrm{~Hz}, 2 \mathrm{H}), 6.67$ (t, $J=7.2 \mathrm{~Hz}, 1 \mathrm{H}), 3.57(\mathrm{tt}, J$ $=11.5,3.5 \mathrm{~Hz}, 1 \mathrm{H}), 2.76(\mathrm{~s}, 3 \mathrm{H}), 1.88-1.75(\mathrm{~m}, 4 \mathrm{H}), 1.71-1.67(\mathrm{~m}, 1 \mathrm{H}), 1.48-$ $1.31(\mathrm{~m}, 4 \mathrm{H}), 1.18-1.08(\mathrm{~m}, 1 \mathrm{H})$.

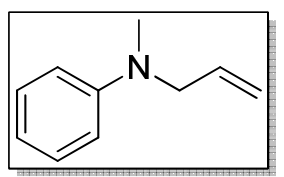

$\boldsymbol{N}$-allyl- $\boldsymbol{N}$-methylaniline (3i) (85\%). ${ }^{\mathbf{1}} \mathbf{H}$ NMR $\left(500 \mathrm{MHz}, \mathrm{CDCl}_{3}\right) \delta=7.24-7.21(\mathrm{~m}$, $2 \mathrm{H}), 6.74-6.69(\mathrm{~m}, 3 \mathrm{H}), 5.88-5.81(\mathrm{~m}, 1 \mathrm{H}), 5.19-5.13(\mathrm{~m}, 2 \mathrm{H}), 3.92(\mathrm{~d}, J=5.1 \mathrm{~Hz}$, $2 \mathrm{H}), 2.94(\mathrm{~s}, 3 \mathrm{H})$.

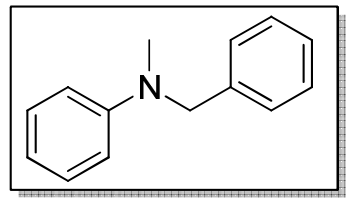

$\boldsymbol{N}$-benzyl- $\boldsymbol{N}$-methylaniline (3j) (87\%). ${ }^{\mathbf{1}} \mathbf{H}$ NMR $\left(500 \mathrm{MHz}, \mathrm{CDCl}_{3}\right) \delta=7.31-7.17$ (m, 7H), $6.75-6.69(\mathrm{~m}, 3 \mathrm{H}), 4.51(\mathrm{~s}, 2 \mathrm{H}), 2.99(\mathrm{~s}, 3 \mathrm{H})$.

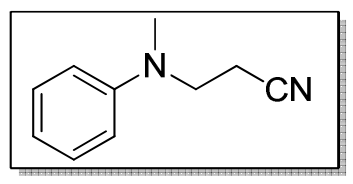

3-(methyl(phenyl)amino)propanenitrile (3k) (56\%). ${ }^{\mathbf{1}} \mathbf{H} \mathbf{N M R}\left(500 \mathrm{MHz}, \mathrm{CDCl}_{3}\right) \delta$ $=7.30-7.27(\mathrm{~m}, 2 \mathrm{H}), 6.79(\mathrm{t}, J=7.3 \mathrm{~Hz}, 1 \mathrm{H}), 6.72(\mathrm{~d}, J=8.0 \mathrm{~Hz}, 2 \mathrm{H}), 3.72(\mathrm{t}, J=$ $6.9 \mathrm{~Hz}, 2 \mathrm{H}), 3.03(\mathrm{~s}, 3 \mathrm{H}), 2.57(\mathrm{t}, J=6.9 \mathrm{~Hz}, 2 \mathrm{H})$. 


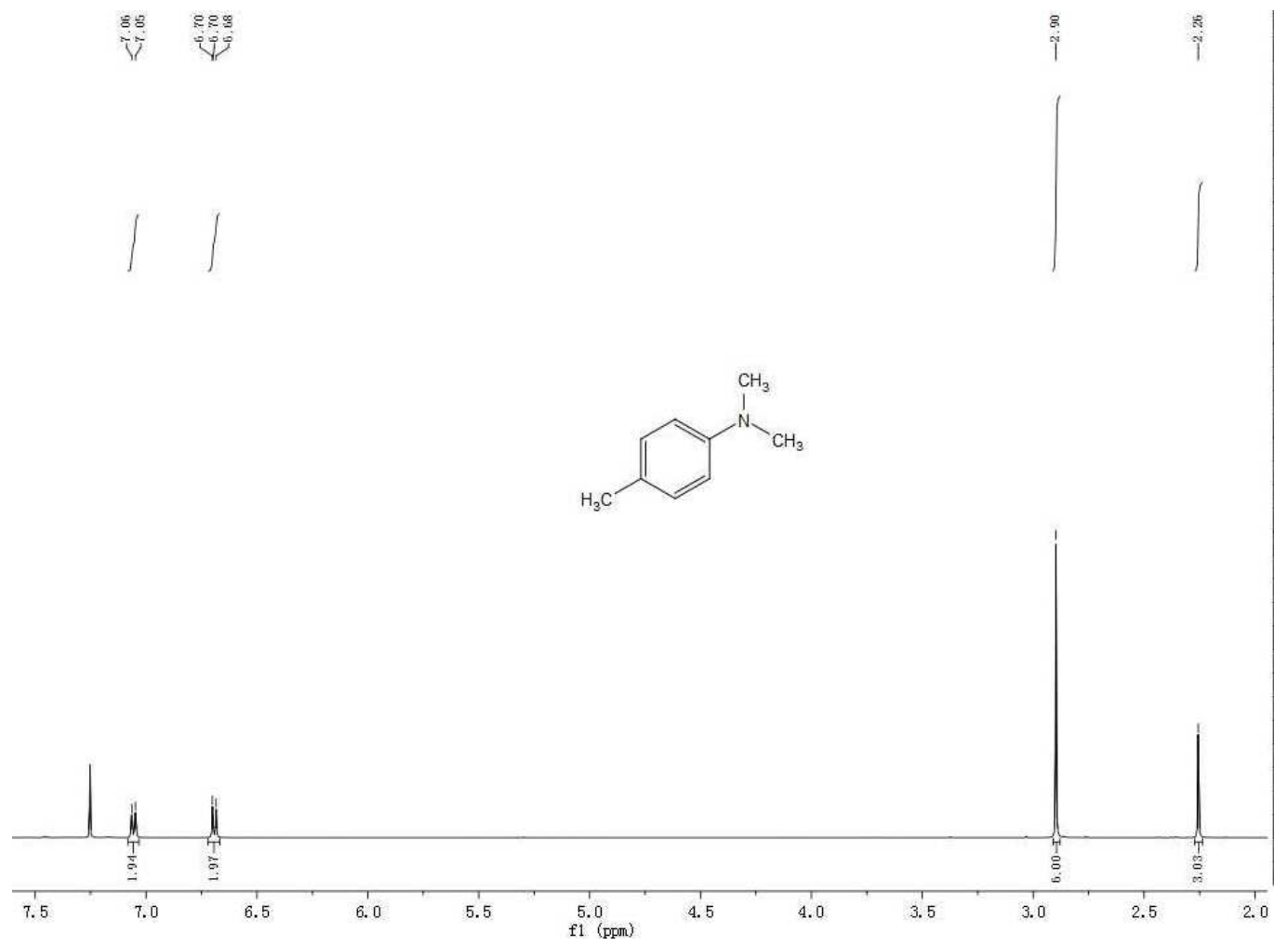

${ }^{1} \mathrm{H}$ NMR spectrum of $\boldsymbol{N}, \boldsymbol{N}, 4$-trimethylaniline (3b) 

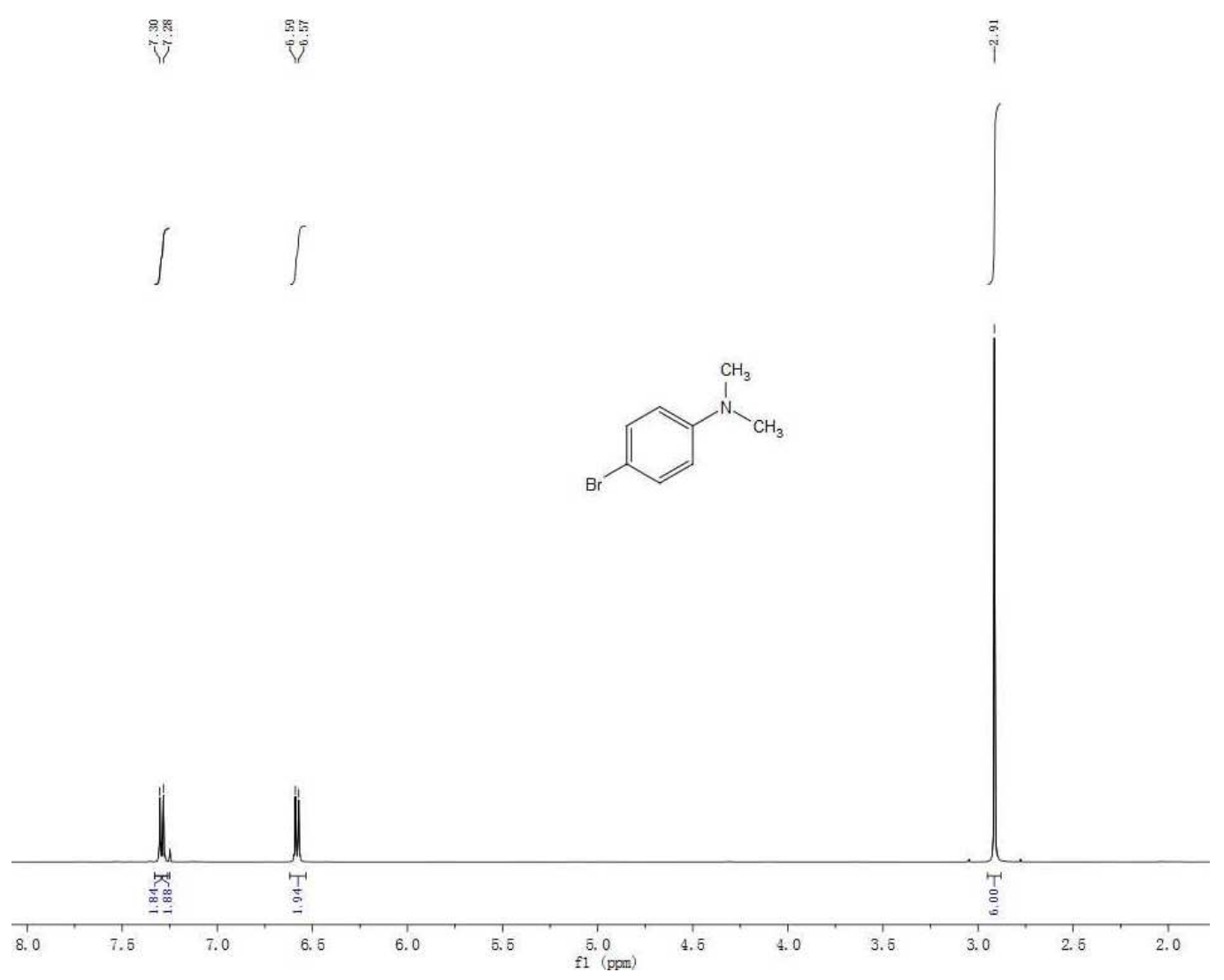

${ }^{1} \mathrm{H}$ NMR spectrum of 4-bromo- $\boldsymbol{N}, \boldsymbol{N}$-dimethylaniline (3d) 


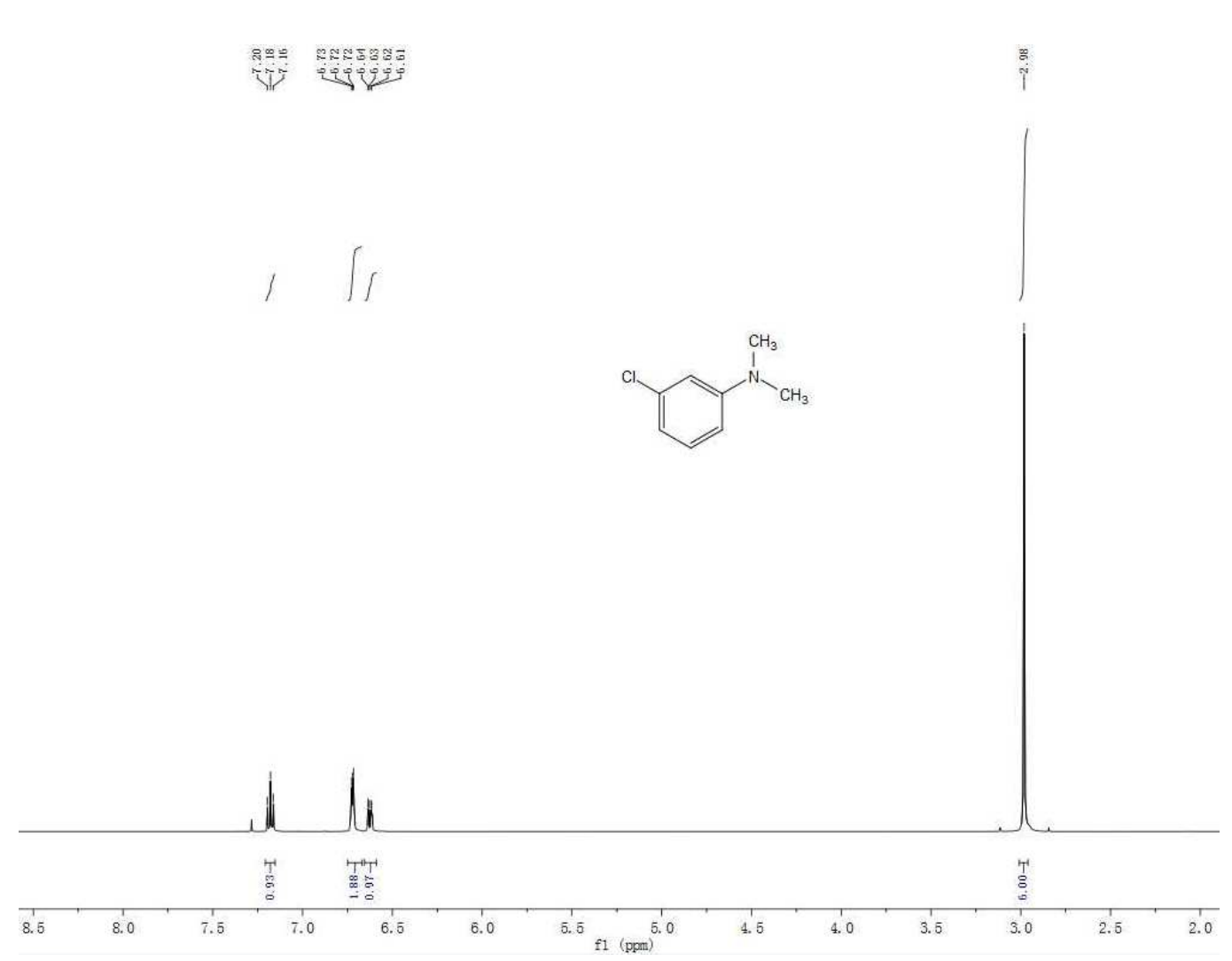

${ }^{1} \mathrm{H}$ NMR spectrum of 3-chloro- $\mathbf{N}, \boldsymbol{N}$-dimethylaniline (3e) 


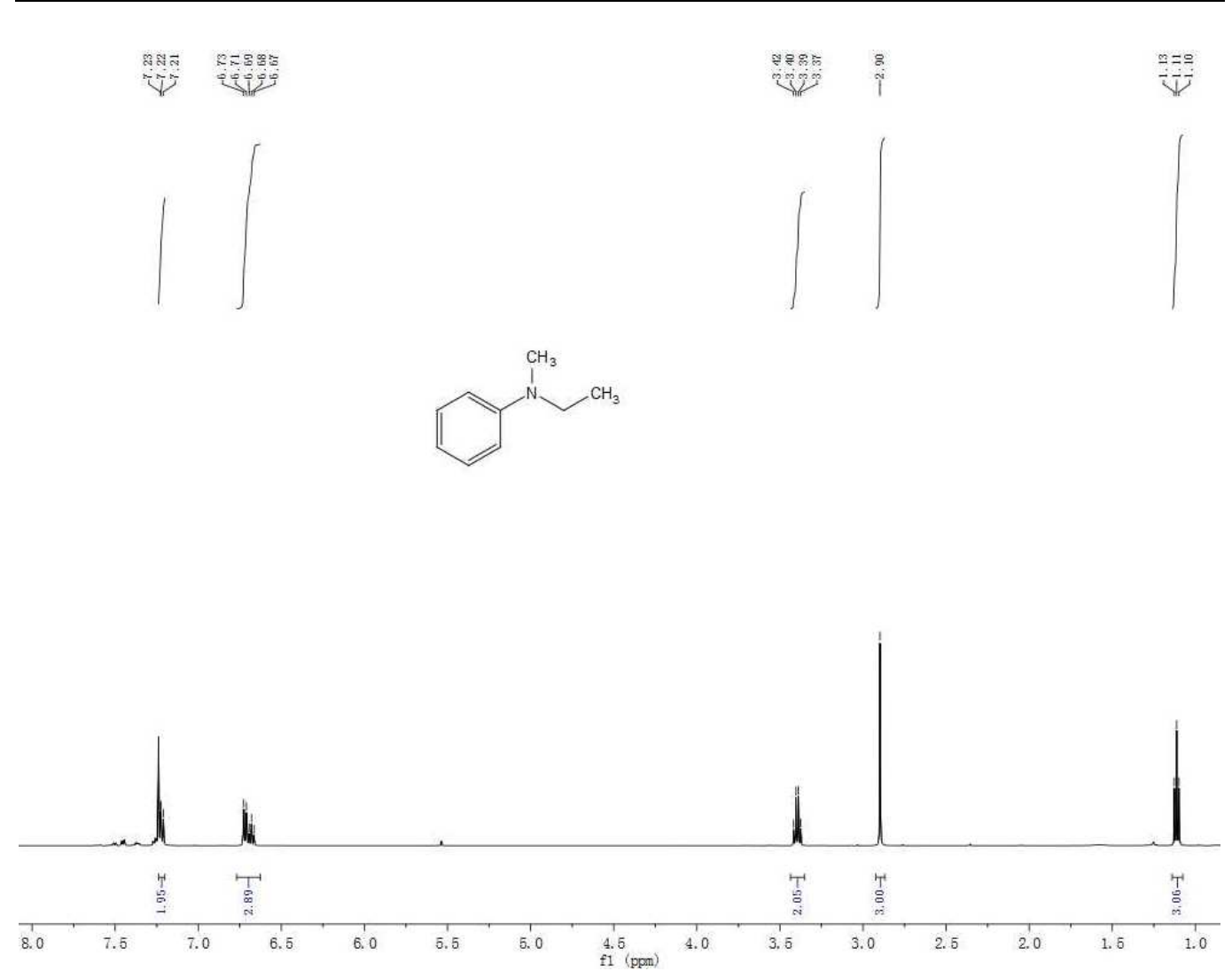

${ }^{1}$ H NMR spectrum of $\boldsymbol{N}$-ethyl- $\boldsymbol{N}$-methylaniline (3g) 


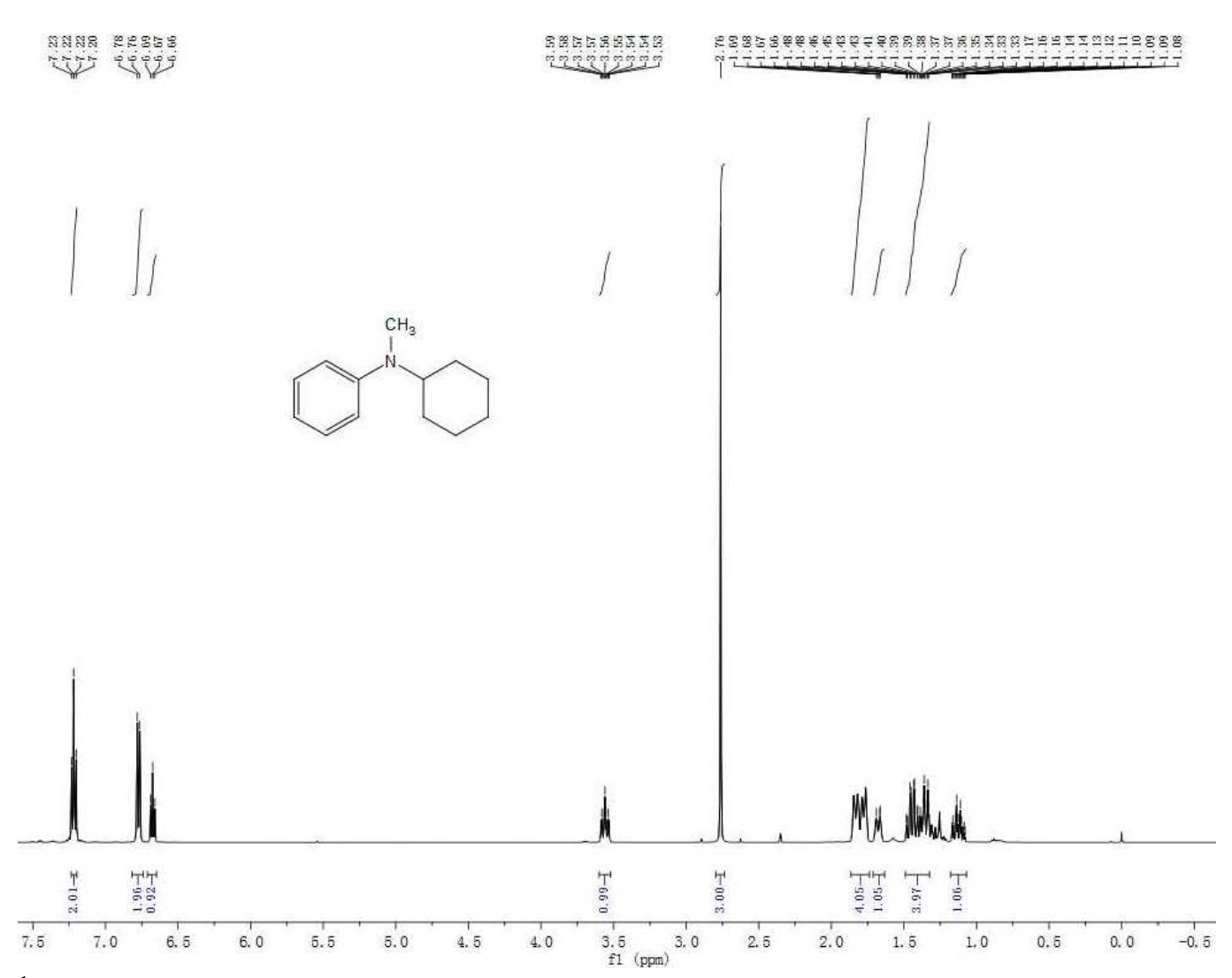

${ }^{1} \mathrm{H}$ NMR spectrum of $\boldsymbol{N}$-cyclohexyl- $\boldsymbol{N}$-methylaniline (3h) 


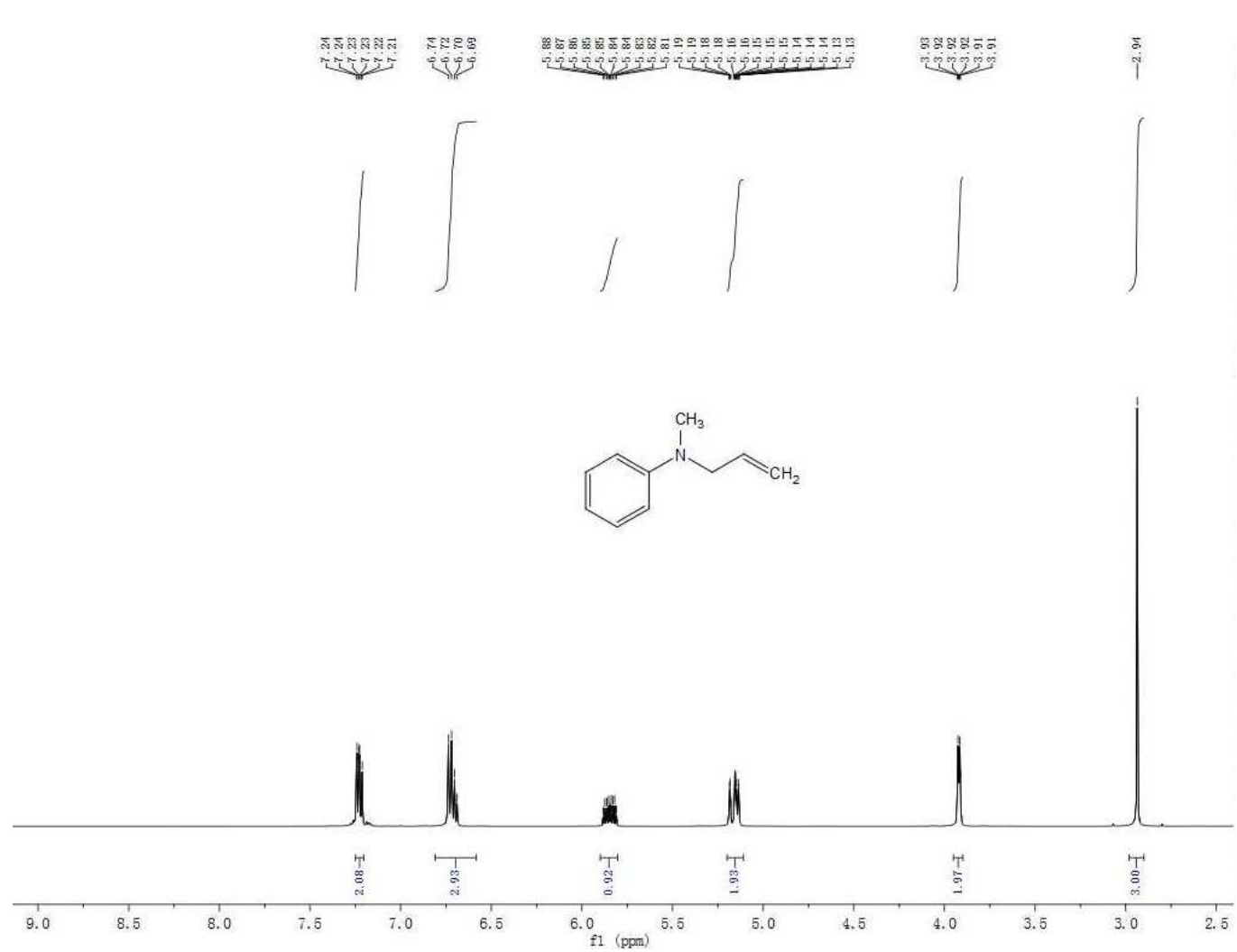

${ }^{1} \mathrm{H}$ NMR spectrum of $\boldsymbol{N}$-allyl- $\boldsymbol{N}$-methylaniline (3i) 


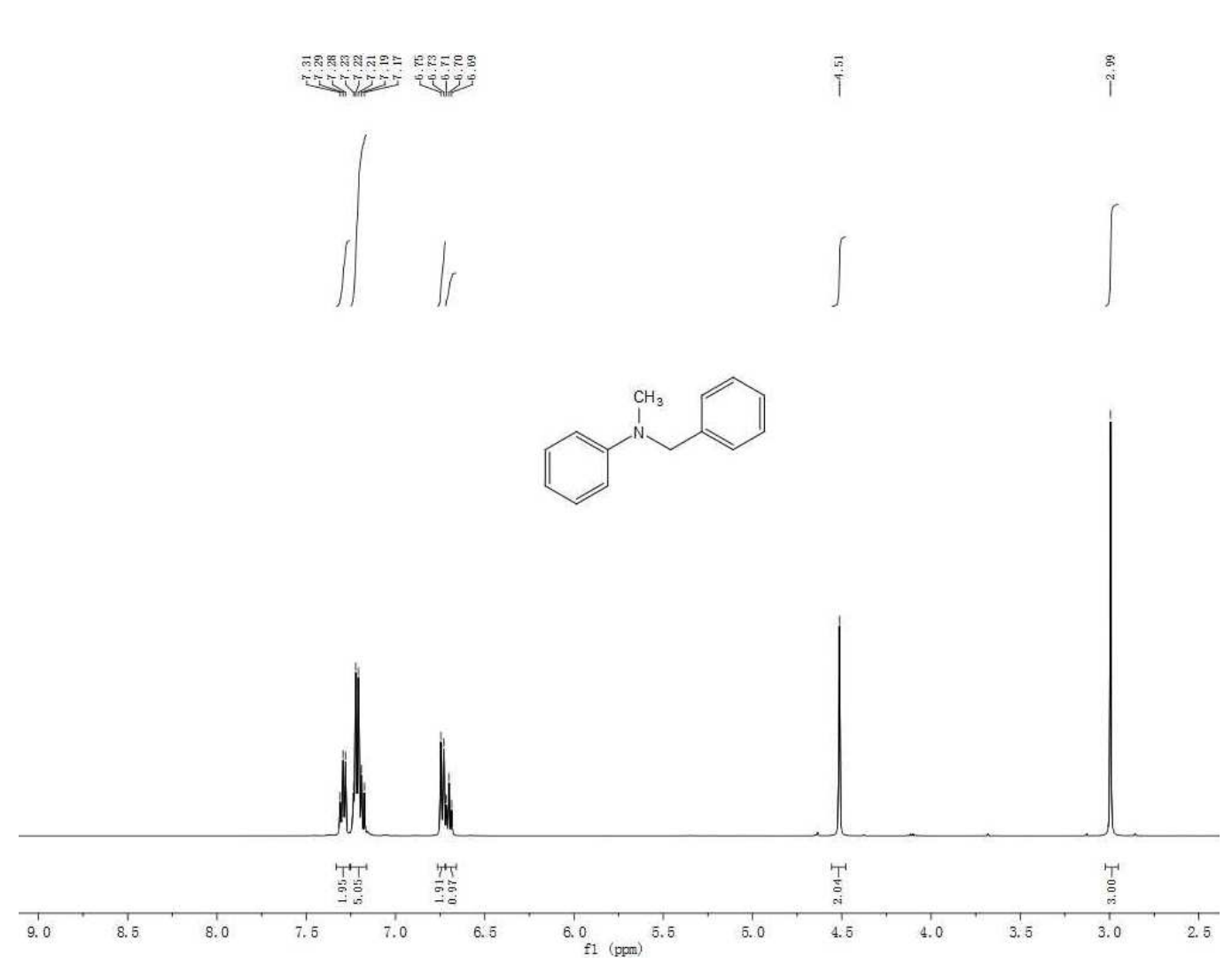

${ }^{1} \mathrm{H}$ NMR spectrum of $\boldsymbol{N}$-benzyl- $\boldsymbol{N}$-methylaniline (3j) 


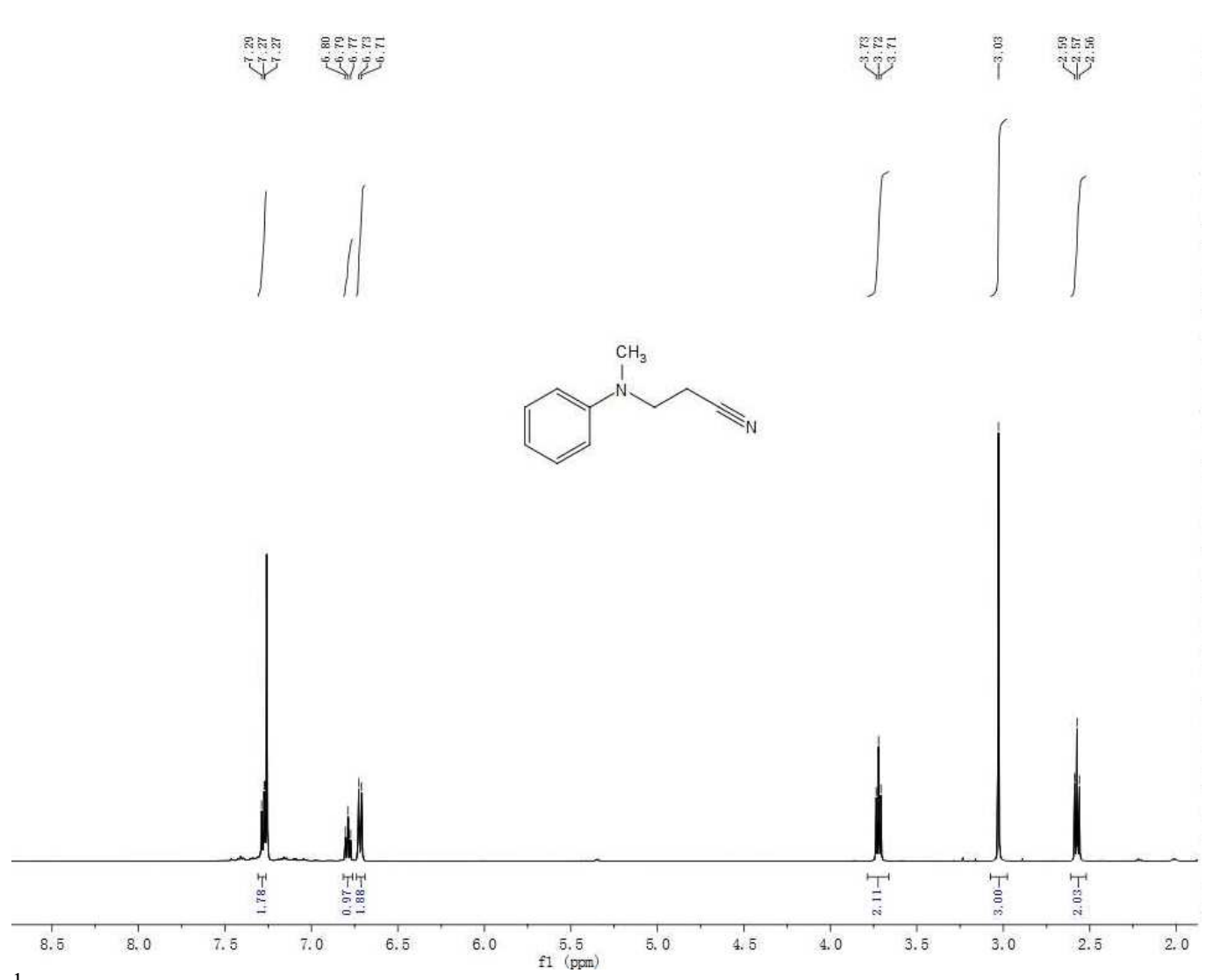

${ }^{1} \mathrm{H}$ NMR spectrum of 3-(methyl(phenyl)amino)propanenitrile (3k) 


\section{Characterization of $N$-methylation of cinacalcet}

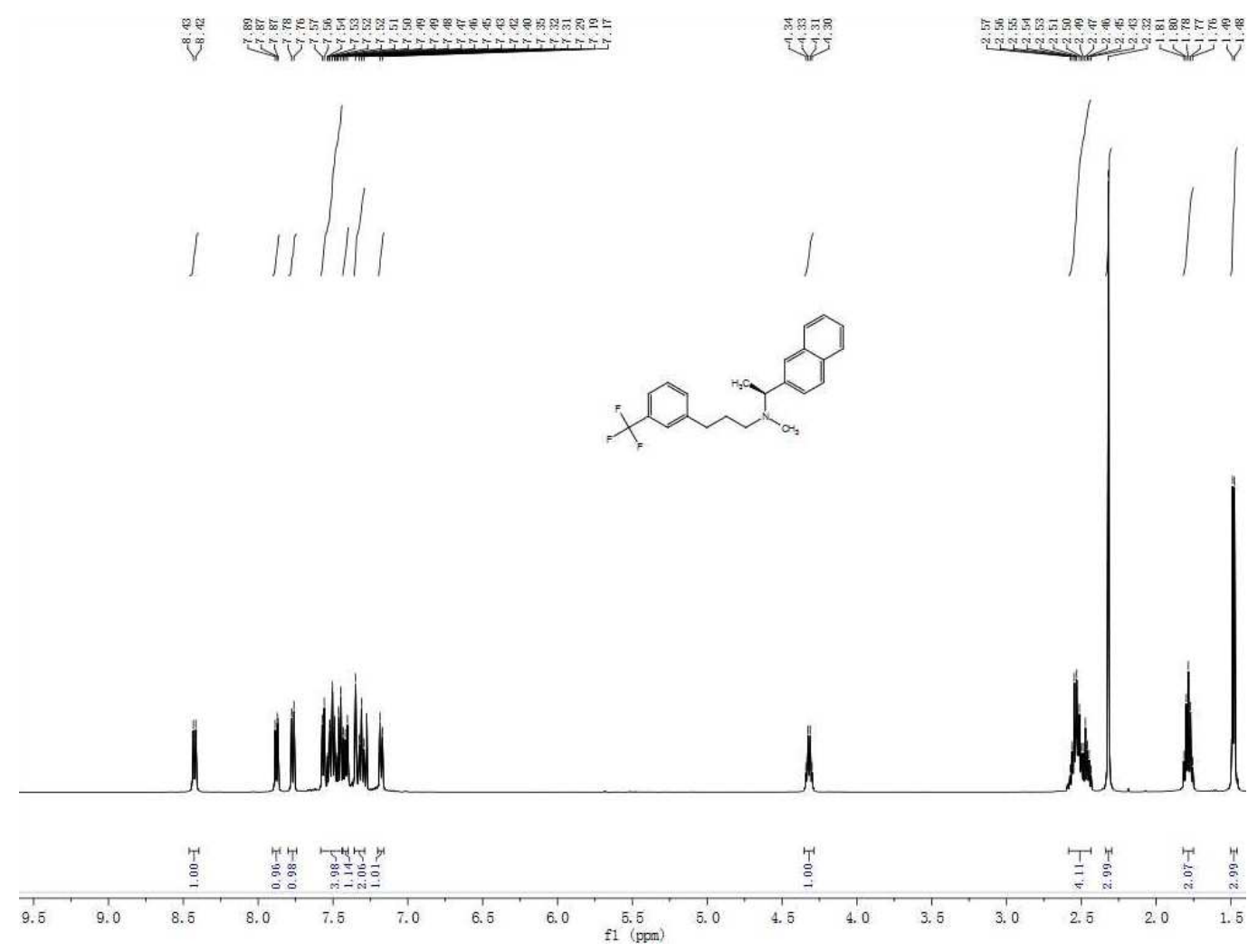

${ }^{1} \mathrm{H}$ NMR $\left(500 \mathrm{MHz}, \mathrm{CDCl}_{3}\right) \delta 8.43(\mathrm{~d}, J=8.2 \mathrm{~Hz}, 1 \mathrm{H}), 7.91-7.85(\mathrm{~m}, 1 \mathrm{H}), 7.77(\mathrm{~d}$, $J=8.1 \mathrm{~Hz}, 1 \mathrm{H}), 7.58-7.44(\mathrm{~m}, 4 \mathrm{H}), 7.44-7.40(\mathrm{~m}, 1 \mathrm{H}), 7.32(\mathrm{dd}, J=17.7,10.0 \mathrm{~Hz}$, 2H), $7.18(\mathrm{~d}, J=7.6 \mathrm{~Hz}, 1 \mathrm{H}), 4.32(\mathrm{q}, J=6.6 \mathrm{~Hz}, 1 \mathrm{H}), 2.61-2.41(\mathrm{~m}, 4 \mathrm{H}), 2.32(\mathrm{~s}$, $3 \mathrm{H}), 1.82-1.75(\mathrm{~m}, 2 \mathrm{H}), 1.48(\mathrm{~d}, J=6.7 \mathrm{~Hz}, 3 \mathrm{H}) .{ }^{1} \mathrm{H}$ NMR data are in accordance with those reported in reference ${ }^{23}$. 


\section{Data for DFT}

$\mathrm{CO}_{2}$

\begin{tabular}{|c|c|c|}
\hline Sum of electronic and zero-point Ene & ies $=$ & -188.578828 \\
\hline Sum of electronic and thermal Energi & & -188.576191 \\
\hline Sum of electronic and thermal Enthal & $\mathrm{es}=$ & -188.575246 \\
\hline Sum of electronic and thermal Free $\mathrm{E}$ & rgies $=$ & -188.599542 \\
\hline Electronic Energy in gas phase $=$ & & -188.5903926 \\
\hline Electronic Energy in acetonitrile= & & -188.5909696 \\
\hline Electronic Energy in tetrahydrofuran= & & -188.5919425 \\
\hline 0.29980657 & 1.47969050 & 0.00000000 \\
\hline-0.95859343 & 1.47969050 & 0.00000000 \\
\hline 1.55820657 & 1.47969050 & 0.00000000 \\
\hline
\end{tabular}

Si-2

Sum of electronic and zero-point Energies=

Sum of electronic and thermal Energies=

Sum of electronic and thermal Enthalpies=

Sum of electronic and thermal Free Energies=

Electronic Energy in gas phase $=$

Electronic Energy in acetonitrile=

Electronic Energy in tetrahydrofuran=

$\begin{array}{lrrr}\mathrm{Si} & 0.32763500 & -1.39180600 & 0.09539300 \\ \mathrm{H} & 0.13066000 & -0.16615900 & 0.95679700 \\ \mathrm{H} & -0.57916400 & -2.49479300 & -0.34451000 \\ \mathrm{C} & 2.16393900 & -1.76968500 & -0.19537500 \\ \mathrm{C} & 2.83253300 & -1.17995900 & -1.28768600 \\ \mathrm{C} & 2.90270900 & -2.64315200 & 0.62791100 \\ \mathrm{C} & 4.18082500 & -1.45432700 & -1.54539500 \\ \mathrm{H} & 2.29301100 & -0.48780000 & -1.92824600 \\ \mathrm{C} & 4.24927000 & -2.92248800 & 0.37018200 \\ \mathrm{H} & 2.41619900 & -3.11031200 & 1.47860300 \\ \mathrm{C} & 4.89422300 & -2.32785500 & -0.71903600 \\ \mathrm{H} & 4.67211100 & -0.98577000 & -2.39625000 \\ \mathrm{H} & 4.79363400 & -3.60609400 & 1.01922000 \\ \mathrm{H} & 5.94098700 & -2.54568400 & -0.92253100 \\ \mathrm{O} & -0.19686800 & -0.47202100 & -1.36223700 \\ \mathrm{C} & -0.14392700 & 0.90095000 & -1.41045500 \\ & & & \mathrm{~S} 52\end{array}$

$-1015.135834$

$-1015.117615$

$-1015.116671$

$-1015.189363$

$-1015.2864343$

$-1015.3570166$

$-1015.346235$

0.09539300 


$\begin{array}{lrrr}\text { Cs } & -3.23403100 & -0.70371600 & -0.48914100 \\ \text { O } & 0.92702400 & 1.44496700 & -1.78330400 \\ \text { O } & -1.18774200 & 1.52193100 & -1.00938700 \\ \text { Cs } & 0.91847300 & 3.11364500 & 0.66209800 \\ \text { O } & 0.25744500 & -2.32489000 & 1.85022900 \\ \text { C } & -0.87474900 & -2.39146800 & 2.45354500 \\ \mathrm{H} & -0.83491700 & -2.93412900 & 3.41825200 \\ \text { O } & -1.96144300 & -1.92026300 & 2.06824600\end{array}$

\section{$\mathrm{Si}-2\left(\mathrm{~K}_{2} \mathrm{CO}_{3}\right)$}

\begin{tabular}{|c|c|c|c|}
\hline \multicolumn{3}{|c|}{ Sum of electronic and zero-point Energies= } & -2175.194453 \\
\hline \multicolumn{3}{|c|}{ Sum of electronic and thermal Energies= } & -2175.176789 \\
\hline \multicolumn{3}{|c|}{ Sum of electronic and thermal Enthalpies= } & -2175.175845 \\
\hline \multicolumn{3}{|c|}{ Sum of electronic and thermal Free Energies= } & -2175.243938 \\
\hline \multicolumn{3}{|c|}{ Electronic Energy in gas phase $=$} & -2175.3461503 \\
\hline \multicolumn{3}{|c|}{ Electronic Energy in acetonitrile $=$} & -2175.3790485 \\
\hline $\mathrm{Si}$ & -0.61614500 & 0.82189100 & -0.14995100 \\
\hline $\mathrm{H}$ & 0.26878200 & 0.80942800 & 1.05747600 \\
\hline $\mathrm{H}$ & -0.56790300 & 1.72764600 & -1.33483400 \\
\hline $\mathrm{C}$ & -2.10332100 & -0.33992300 & -0.08857400 \\
\hline $\mathrm{C}$ & -1.96765400 & -1.67872200 & -0.50529900 \\
\hline $\mathrm{C}$ & -3.37282300 & 0.08608800 & 0.34896300 \\
\hline $\mathrm{C}$ & -3.05842700 & -2.55421000 & -0.48769300 \\
\hline $\mathrm{H}$ & -0.99641000 & -2.03974100 & -0.83048300 \\
\hline $\mathrm{C}$ & -4.46555000 & -0.78625100 & 0.36263400 \\
\hline $\mathrm{H}$ & -3.50642300 & 1.11047100 & 0.68228600 \\
\hline $\mathrm{C}$ & -4.31174900 & -2.11089900 & -0.05661700 \\
\hline $\mathrm{H}$ & -2.92696700 & -3.58454100 & -0.81259800 \\
\hline $\mathrm{H}$ & -5.43660400 & -0.43079000 & 0.70174000 \\
\hline $\mathrm{H}$ & -5.16117700 & -2.79104300 & -0.04643200 \\
\hline $\mathrm{O}$ & 0.66984500 & -0.16683600 & -1.04270400 \\
\hline $\mathrm{C}$ & 1.61855800 & -0.93847000 & -0.49113000 \\
\hline K & 2.20854200 & 2.16843400 & -0.75742400 \\
\hline $\mathrm{O}$ & 1.38287500 & -2.15268000 & -0.23609800 \\
\hline $\mathrm{O}$ & 2.76320400 & -0.37565100 & -0.25639300 \\
\hline K & 3.57311900 & -2.52129600 & 0.94044200 \\
\hline $\mathrm{O}$ & -1.50753600 & 2.32314900 & 0.74212200 \\
\hline $\mathrm{C}$ & -0.81864700 & 3.39769300 & 0.89477400 \\
\hline $\mathrm{H}$ & -1.36576900 & 4.21295800 & 1.40220300 \\
\hline $\mathrm{O}$ & 0.35969100 & 3.58847700 & 0.53560800 \\
\hline
\end{tabular}




\section{$\mathrm{PhSiH}_{3}$}

\begin{tabular}{|c|c|c|c|}
\hline \multicolumn{3}{|c|}{ Sum of electronic and zero-point Energies= } & -522.837779 \\
\hline \multicolumn{3}{|c|}{ Sum of electronic and thermal Energies $=$} & -522.830736 \\
\hline \multicolumn{3}{|c|}{ Sum of electronic and thermal Enthalpies= } & -522.829792 \\
\hline \multicolumn{3}{|c|}{ Sum of electronic and thermal Free Energies= } & -522.870283 \\
\hline \multicolumn{3}{|c|}{ Electronic Energy in gas phase $=$} & -522.953261 \\
\hline \multicolumn{3}{|c|}{ Electronic Energy in acetonitrile $=$} & -522.9603985 \\
\hline \multicolumn{3}{|c|}{ Electronic Energy in tetrahydrofuran= } & -522.9597787 \\
\hline $\mathrm{Si}$ & 2.35164800 & 0.00000200 & 0.00616400 \\
\hline $\mathrm{H}$ & 2.86495500 & -1.21431900 & -0.68230700 \\
\hline $\mathrm{H}$ & 2.86473900 & 1.21620500 & -0.67912200 \\
\hline $\mathrm{H}$ & 2.88323800 & -0.00183500 & 1.39734300 \\
\hline $\mathrm{C}$ & 0.46973000 & -0.00000500 & -0.01425400 \\
\hline $\mathrm{C}$ & -0.25660000 & 1.20569700 & -0.01043300 \\
\hline $\mathrm{C}$ & -0.25661100 & -1.20570600 & -0.01043200 \\
\hline $\mathrm{C}$ & -1.65421200 & 1.20874300 & 0.00325500 \\
\hline $\mathrm{H}$ & 0.27102800 & 2.15762200 & -0.02330100 \\
\hline $\mathrm{C}$ & -1.65421500 & -1.20874400 & 0.00325700 \\
\hline $\mathrm{H}$ & 0.27100000 & -2.15764400 & -0.02330000 \\
\hline $\mathrm{C}$ & -2.35591800 & 0.00000500 & 0.01133200 \\
\hline $\mathrm{H}$ & -2.19397000 & 2.15268900 & 0.00422900 \\
\hline $\mathrm{H}$ & -2.19398100 & -2.15268500 & 0.00423200 \\
\hline $\mathrm{H}$ & -3.44312500 & 0.00000600 & 0.01959300 \\
\hline
\end{tabular}

\section{TS-1}

\begin{tabular}{|c|c|c|c|}
\hline \multicolumn{3}{|c|}{ Sum of electronic and zero-point Energies= } & -1015.079599 \\
\hline \multicolumn{3}{|c|}{ Sum of electronic and thermal Energies= } & -1015.060534 \\
\hline \multicolumn{3}{|c|}{ Sum of electronic and thermal Enthalpies= } & -1015.059589 \\
\hline \multicolumn{3}{|c|}{ Sum of electronic and thermal Free Energies= } & -1015.137686 \\
\hline \multicolumn{3}{|c|}{ Electronic Energy in gas phase $=$} & -1015.2232004 \\
\hline \multicolumn{3}{|c|}{ Electronic Energy in acetonitrile $=$} & -1015.3036966 \\
\hline \multicolumn{3}{|c|}{ Electronic Energy in tetrahydrofuran= } & -1015.2919884 \\
\hline $\mathrm{Si}$ & 1.24457500 & 1.71556700 & 0.29696400 \\
\hline $\mathrm{H}$ & 0.80252000 & 2.15205200 & 1.65153300 \\
\hline $\mathrm{H}$ & 2.33190000 & 2.92107500 & 0.23221700 \\
\hline $\mathrm{H}$ & 0.68796400 & 2.15834800 & -1.01518700 \\
\hline $\mathrm{C}$ & 2.66645500 & 0.42479500 & 0.23930400 \\
\hline $\mathrm{C}$ & 3.09395300 & -0.25683100 & 1.39739500 \\
\hline
\end{tabular}




$\begin{array}{lrrr}\mathrm{C} & 3.31115600 & 0.08958800 & -0.96900000 \\ \mathrm{C} & 4.09912400 & -1.23264000 & 1.35458100 \\ \mathrm{H} & 2.63460400 & -0.01833600 & 2.35559100 \\ \mathrm{C} & 4.30968800 & -0.89163500 & -1.02693000 \\ \mathrm{H} & 3.03898100 & 0.61648900 & -1.88179000 \\ \mathrm{C} & 4.70527300 & -1.56444600 & 0.13627200 \\ \mathrm{H} & 4.41858200 & -1.72399000 & 2.27245600 \\ \mathrm{H} & 4.79337000 & -1.11677900 & -1.97598800 \\ \mathrm{H} & 5.49387700 & -2.31314300 & 0.09860400 \\ \mathrm{O} & 0.05431200 & 0.22695800 & 0.31566100 \\ \mathrm{C} & -1.28107700 & 0.30916500 & 0.25586000 \\ \mathrm{Cs} & 0.66178800 & -2.58377200 & -0.17040300 \\ \mathrm{O} & -1.84952400 & 1.43286100 & 0.37345600 \\ \mathrm{O} & -1.91087600 & -0.80974200 & 0.06839800 \\ \mathrm{Cs} & -4.60118900 & 0.61166300 & -0.05596500 \\ \mathrm{C} & 3.49160100 & 4.14240600 & -0.30491000 \\ \mathrm{O} & 3.63108500 & 4.77572600 & 0.69047300 \\ \mathrm{O} & 3.65802200 & 3.83043900 & -1.44050700 \\ & & & \end{array}$

\section{$\mathrm{TS}-1\left(\mathrm{~K}_{2} \mathrm{CO}_{3}\right)$}

\begin{tabular}{|c|c|c|c|}
\hline Sun & d zero-point En & gies $=$ & -2175.138130 \\
\hline Sun & $d$ thermal Energ & & -2175.119648 \\
\hline Sun & d thermal Entha & ies $=$ & -2175.118704 \\
\hline Sun & d thermal Free & nergies= & -2175.191442 \\
\hline Ele & gas phase $=$ & & -2175.2827663 \\
\hline Ele & acetonitrile $=$ & & -2175.3247069 \\
\hline $\mathrm{Si}$ & -0.57058100 & 1.23275100 & 0.41094200 \\
\hline $\mathrm{H}$ & -0.08973000 & 1.87251100 & -0.84234600 \\
\hline $\mathrm{H}$ & -1.93885300 & 2.13232500 & 0.33026900 \\
\hline $\mathrm{H}$ & -0.28035500 & 1.73039600 & 1.78051000 \\
\hline $\mathrm{C}$ & -1.59163200 & -0.38065700 & 0.24793800 \\
\hline $\mathrm{C}$ & -2.13385400 & -0.78289000 & -0.98929700 \\
\hline $\mathrm{C}$ & -1.75279900 & -1.26226600 & 1.33680400 \\
\hline $\mathrm{C}$ & -2.77102400 & -2.02157000 & -1.14307500 \\
\hline $\mathrm{H}$ & -2.07264900 & -0.11155800 & -1.84301400 \\
\hline $\mathrm{C}$ & -2.39956100 & -2.49727300 & 1.19612700 \\
\hline $\mathrm{H}$ & -1.36304800 & -0.98222800 & 2.31414300 \\
\hline $\mathrm{C}$ & -2.89972400 & -2.88938500 & -0.05192500 \\
\hline $\mathrm{H}$ & -3.18419900 & -2.30000200 & -2.11049600 \\
\hline $\mathrm{H}$ & -2.52316800 & -3.14692000 & 2.06076000 \\
\hline $\mathrm{H}$ & -3.40750300 & -3.84450500 & -0.16567800 \\
\hline
\end{tabular}




$\begin{array}{lrrr}\mathrm{O} & 0.95209200 & 0.06028700 & 0.42539900 \\ \mathrm{C} & 2.23680900 & 0.32789800 & 0.21012000 \\ \mathrm{~K} & 0.85464900 & -2.36254400 & -0.29961800 \\ \mathrm{O} & 2.69238700 & 1.50000900 & 0.32435000 \\ \mathrm{O} & 2.96988400 & -0.69806200 & -0.12476100 \\ \mathrm{~K} & 5.04691900 & 0.84526000 & -0.24536600 \\ \mathrm{C} & -3.21340500 & 2.91533400 & -0.23497800 \\ \mathrm{O} & -3.32084000 & 2.52480400 & -1.36225100 \\ \mathrm{O} & -3.55989900 & 3.58355300 & 0.69314700\end{array}$

\section{TS-1'}

\begin{tabular}{|c|c|c|c|}
\hline \multicolumn{3}{|c|}{ Sum of electronic and zero-point Energies= } & -1015.075763 \\
\hline \multicolumn{3}{|c|}{ Sum of electronic and thermal Energies= } & -1015.057053 \\
\hline \multicolumn{3}{|c|}{ Sum of electronic and thermal Enthalpies= } & -1015.056109 \\
\hline \multicolumn{3}{|c|}{ Sum of electronic and thermal Free Energies= } & -1015.131547 \\
\hline \multicolumn{3}{|c|}{ Electronic Energy in gas phase $=$} & -1015.2194806 \\
\hline \multicolumn{3}{|c|}{ Electronic Energy in acetonitrile= } & -1015.2957628 \\
\hline $\mathrm{Si}$ & 1.63964200 & -0.85629000 & 0.06734000 \\
\hline $\mathrm{H}$ & 1.22161100 & -1.63620200 & -1.13487000 \\
\hline $\mathrm{H}$ & 1.31374400 & -1.16811700 & 1.49406300 \\
\hline $\mathrm{H}$ & 2.29028000 & 0.58856700 & -0.23876300 \\
\hline $\mathrm{C}$ & 3.46055000 & -1.58252200 & 0.14686800 \\
\hline $\mathrm{C}$ & 4.18457100 & -1.61560200 & 1.35291100 \\
\hline $\mathrm{C}$ & 4.10228900 & -2.10226300 & -0.99161700 \\
\hline $\mathrm{C}$ & 5.48333100 & -2.13236900 & 1.42538900 \\
\hline $\mathrm{H}$ & 3.71921000 & -1.23715600 & 2.26443500 \\
\hline $\mathrm{C}$ & 5.39941300 & -2.62521100 & -0.93743900 \\
\hline $\mathrm{H}$ & 3.57517700 & -2.10284600 & -1.94615800 \\
\hline $\mathrm{C}$ & 6.09704800 & -2.63991800 & 0.27498100 \\
\hline $\mathrm{H}$ & 6.01480300 & -2.14463800 & 2.37597000 \\
\hline $\mathrm{H}$ & 5.86700300 & -3.01963500 & -1.83839500 \\
\hline $\mathrm{H}$ & 7.10603000 & -3.04476700 & 0.32356900 \\
\hline $\mathrm{O}$ & 0.06730300 & 0.21335800 & -0.04632300 \\
\hline $\mathrm{C}$ & -1.14218200 & -0.35872000 & 0.07047100 \\
\hline Cs & -0.65549700 & 3.08993300 & 0.20201900 \\
\hline $\mathrm{O}$ & -1.23323800 & -1.61095400 & 0.22685600 \\
\hline $\mathrm{O}$ & -2.16183600 & 0.43887600 & 0.01148500 \\
\hline Cs & -4.10918000 & -1.89379300 & -0.10107300 \\
\hline $\mathrm{C}$ & 3.04496900 & 1.90803700 & -0.72766400 \\
\hline $\mathrm{O}$ & 2.32265600 & 2.80781400 & -0.37389100 \\
\hline $\mathrm{O}$ & 4.03191800 & 1.50797300 & -1.25288900 \\
\hline
\end{tabular}




\section{$\mathrm{Si}-3$}

Sum of electronic and zero-point Energies=

Sum of electronic and thermal Energies=

Sum of electronic and thermal Enthalpies=

Sum of electronic and thermal Free Energies=

Electronic Energy in gas phase=

Electronic Energy in acetonitrile=

Electronic Energy in tetrahydrofuran=

$\begin{array}{lrrr}\mathrm{Si} & 0.97261600 & -0.34654300 & 1.15699200 \\ \mathrm{H} & 1.31337100 & 0.82233000 & 2.00945200 \\ \mathrm{H} & 1.09468200 & -1.62261100 & 1.89337400 \\ \mathrm{C} & -0.72734300 & -0.12331000 & 0.42636000 \\ \mathrm{C} & -1.40548800 & -1.19833900 & -0.18149100 \\ \mathrm{C} & -1.37148500 & 1.12779100 & 0.47396100 \\ \mathrm{C} & -2.67785000 & -1.02678100 & -0.72977200 \\ \mathrm{H} & -0.93758700 & -2.17956900 & -0.23058600 \\ \mathrm{C} & -2.64608500 & 1.30267600 & -0.07156500 \\ \mathrm{H} & -0.87773000 & 1.97650700 & 0.94335900 \\ \mathrm{C} & -3.29932500 & 0.22497800 & -0.67506100 \\ \mathrm{H} & -3.18489400 & -1.86709900 & -1.19703700 \\ \mathrm{H} & -3.12747000 & 2.27619400 & -0.02484100 \\ \mathrm{H} & -4.29099000 & 0.35865600 & -1.09995800 \\ \mathrm{O} & 2.15701200 & -0.45176400 & -0.06613800 \\ \mathrm{C} & 3.05353700 & 0.47897000 & -0.44763800 \\ \mathrm{H} & 3.02716000 & 1.39961200 & 0.16352600 \\ \mathrm{O} & 3.81937200 & 0.32322300 & -1.36185500\end{array}$

$-711.428764$

$-711.418699$

$-711.417754$

$-711.467340$

$-711.5609717$

$-711.5764125$

$-711.5750226$

15699200

\section{$\mathrm{CsH}$}

Sum of electronic and zero-point Energies=

Sum of electronic and thermal Energies=

Sum of electronic and thermal Enthalpies=

Sum of electronic and thermal Free Energies=

Electronic Energy in gas phase $=$

Electronic Energy in acetonitrile=

Cs

$\mathrm{H}$
0.00000000

0.00000000

0.00000000
$-20.426385$

$-20.423959$

$-20.423015$

$-20.447578$

$-20.4283178$

$-20.4937719$

0.04841200

$-2.66265600$ 


\section{Cs-1}

Sum of electronic and zero-point Energies=

Sum of electronic and thermal Energies=

Sum of electronic and thermal Enthalpies=

Sum of electronic and thermal Free Energies=

Electronic Energy in gas phase=

Electronic Energy in acetonitrile=

$\begin{array}{lrrr}\mathrm{Si} & 1.71353100 & -0.92052600 & 0.00017900 \\ \mathrm{H} & 1.49121000 & -1.75045200 & -1.21251300 \\ \mathrm{H} & 1.49123500 & -1.75006000 & 1.21314400 \\ \mathrm{C} & 3.49769100 & -0.30701700 & 0.00003900 \\ \mathrm{C} & 4.56587700 & -1.22412700 & -0.00016700 \\ \mathrm{C} & 3.81088500 & 1.06474000 & 0.00016200 \\ \mathrm{C} & 5.89583000 & -0.79397900 & -0.00023600 \\ \mathrm{H} & 4.36320900 & -2.29520000 & -0.00028200 \\ \mathrm{C} & 5.13997600 & 1.50243200 & 0.00009400 \\ \mathrm{H} & 3.00433800 & 1.79324800 & 0.00030300 \\ \mathrm{C} & 6.18548300 & 0.57465200 & -0.00010300 \\ \mathrm{H} & 6.70343500 & -1.52281700 & -0.00039700 \\ \mathrm{H} & 5.35835600 & 2.56817300 & 0.00019100 \\ \mathrm{H} & 7.21881400 & 0.91427000 & -0.00015700 \\ \mathrm{O} & 0.80722600 & 0.50447400 & -0.00001400 \\ \mathrm{C} & -0.57520300 & 0.36518300 & 0.00001400 \\ \mathrm{O} & -1.24981400 & 1.41729300 & -0.00014800 \\ \mathrm{O} & -0.99762300 & -0.83376700 & 0.00020000 \\ \mathrm{Cs} & -3.87675600 & -0.01572900 & -0.00003500\end{array}$

\section{$\mathrm{Cs}_{2} \mathrm{CO}_{3}$}

Sum of electronic and zero-point Energies=

Sum of electronic and thermal Energies=

Sum of electronic and thermal Enthalpies=

Sum of electronic and thermal Free Energies=

Electronic Energy in gas phase=

Electronic Energy in acetonitrile=

Electronic Energy in tetrahydrofuran=

$\begin{array}{lrrr}\mathrm{C} & -0.00001400 & 1.25486900 & 0.00001400 \\ \mathrm{O} & 1.12918600 & 1.87455900 & 0.00039000 \\ \mathrm{O} & -1.12922800 & 1.87457900 & -0.00040300\end{array}$

$-806.031012$

$-806.018433$

$-806.017489$

$-806.075372$

$-806.156767$

$-806.207588$

$-303.651100$

$-303.643786$

$-303.642841$

$-303.688589$

$-303.666576$

$-303.7526518$

$-303.7364974$ 


$\begin{array}{lrrr}\text { O } & -0.00000100 & -0.09128400 & 0.00005400 \\ \text { Cs } & 2.94418200 & -0.33447000 & -0.00002600 \\ \text { Cs } & -2.94417400 & -0.33447600 & 0.00001800\end{array}$

\section{$\mathrm{K}_{2} \mathrm{CO}_{3}$}

Sum of electronic and zero-point Energies $=$

Sum of electronic and thermal Energies=

Sum of electronic and thermal Enthalpies=

Sum of electronic and thermal Free Energies=

Electronic Energy in gas phase $=$

Electronic Energy in acetonitrile=

$\mathrm{O}$

$\mathrm{O}$

$\mathrm{O}$

K

K

$\begin{array}{rrr}0.00000000 & 0.80975000 & 0.00000300 \\ 1.13029200 & 1.41894000 & 0.00022900 \\ -1.13029300 & 1.41894000 & -0.00025000 \\ 0.00000000 & -0.54250700 & 0.00002900 \\ 2.52452000 & -0.61109200 & 0.00004500 \\ -2.52451900 & -0.61109200 & -0.00004900\end{array}$

\section{Si-1}

Sum of electronic and zero-point Energies $=$

Sum of electronic and thermal Energies=

Sum of electronic and thermal Enthalpies=

Sum of electronic and thermal Free Energies=

Electronic Energy in gas phase $=$

Electronic Energy in acetonitrile=

Electronic Energy in tetrahydrofuran=
$-1463.716012$

$-1463.709257$

$-1463.708313$

$-1463.749139$

$-1463.7324279$

$-1463.7732307$

$-826.503877$

$-826.488271$

$-826.487327$

$-826.554197$

$-826.6360567$

$-826.714999$

$-826.701496$

$-0.00057200$

1.33181200

$-0.00103600$

$-1.33307100$

$-0.00003500$

1.19881600

$-1.19840400$

1.20744700

2.15121300

$-1.20612800$

$-2.15114000$

0.00088700 


$\begin{array}{lrrr}\mathrm{H} & 5.02166800 & 0.17453800 & 2.15479100 \\ \mathrm{H} & 5.02283800 & 0.17380900 & -2.15312400 \\ \mathrm{H} & 6.06389200 & -0.53894000 & 0.00123500 \\ \mathrm{O} & 0.27633500 & 0.84875700 & -0.00066500 \\ \mathrm{C} & -1.02318000 & 0.59810100 & -0.00045300 \\ \mathrm{Cs} & 1.50478300 & -1.78510000 & -0.00014700 \\ \mathrm{O} & -1.86818900 & 1.54723500 & 0.00009400 \\ \mathrm{O} & -1.37596400 & -0.66261700 & -0.00075500 \\ \mathrm{Cs} & -4.31116100 & 0.00157700 & 0.00024100\end{array}$

\section{$\mathrm{Si}-1\left(\mathrm{~K}_{2} \mathrm{CO}_{3}\right)$}

Sum of electronic and zero-point Energies=

Sum of electronic and thermal Energies=

Sum of electronic and thermal Enthalpies=

Sum of electronic and thermal Free Energies=

Electronic Energy in gas phase=

Electronic Energy in acetonitrile=

\begin{tabular}{|c|c|c|c|}
\hline \multicolumn{3}{|c|}{ Electronic Energy in gas phase $=$} & -1986.6989798 \\
\hline \multicolumn{3}{|c|}{ Electronic Energy in acetonitrile $=$} & -1986.7346624 \\
\hline $\mathrm{Si}$ & 0.78464800 & 2.40210400 & -0.00053200 \\
\hline $\mathrm{H}$ & 0.08564700 & 2.60880600 & 1.28940900 \\
\hline $\mathrm{H}$ & 1.74285700 & 3.58835600 & -0.00067800 \\
\hline $\mathrm{H}$ & 0.08601400 & 2.60821600 & -1.29077200 \\
\hline $\mathrm{C}$ & 2.05979400 & 0.97784900 & 0.00001300 \\
\hline $\mathrm{C}$ & 2.54149700 & 0.42036200 & 1.20069900 \\
\hline $\mathrm{C}$ & 2.54189500 & 0.41980500 & -1.20025600 \\
\hline $\mathrm{C}$ & 3.43746400 & -0.65684400 & 1.20815800 \\
\hline $\mathrm{H}$ & 2.20206300 & 0.82580500 & 2.15230400 \\
\hline $\mathrm{C}$ & 3.43786800 & -0.65740400 & -1.20691900 \\
\hline $\mathrm{H}$ & 2.20278300 & 0.82481100 & -2.15216200 \\
\hline $\mathrm{C}$ & 3.88555000 & -1.20780100 & 0.00082200 \\
\hline $\mathrm{H}$ & 3.79468900 & -1.05870800 & 2.15471300 \\
\hline $\mathrm{H}$ & 3.79541400 & -1.05970100 & -2.15317000 \\
\hline $\mathrm{H}$ & 4.59026900 & -2.03652100 & 0.00113200 \\
\hline $\mathrm{O}$ & -0.55471300 & 0.49833600 & -0.00038400 \\
\hline $\mathrm{C}$ & -1.82985000 & 0.24621400 & -0.00022900 \\
\hline K & 0.31897700 & -1.83487200 & 0.00011800 \\
\hline $\mathrm{O}$ & -2.71358000 & 1.16789000 & -0.00008100 \\
\hline $\mathrm{O}$ & -2.18848500 & -1.03350400 & -0.00021900 \\
\hline K & -4.64928800 & -0.38857000 & -0.00020200 \\
\hline
\end{tabular}

$-1986.565943$

$-1986.551474$

$-1986.550529$

$-1986.610966$

$-1986.6989798$

$-1986.7346624$

0.00053200 
Si-1'

Sum of electronic and zero-point Energies=

Sum of electronic and thermal Energies=

Sum of electronic and thermal Enthalpies=

Sum of electronic and thermal Free Energies=

Electronic Energy in gas phase=

Electronic Energy in acetonitrile=

\section{$\mathrm{Si}$}

$\mathrm{H}$

$\mathrm{H}$

$\mathrm{H}$

C

C

C

C

$\mathrm{H}$

C

$\mathrm{H}$

C

$\mathrm{H}$

$\mathrm{H}$

$\mathrm{H}$

$\mathrm{O}$

C

Cs

$\mathrm{O}$

O

Cs

$\begin{array}{ccc}2.10778800 & -0.35456500 & -0.00054100 \\ 1.78671500 & -0.95559400 & -1.33344500 \\ 1.78593800 & -0.95674400 & 1.33166600 \\ 2.46628400 & 1.12065900 & 0.00008600 \\ 3.99334100 & -0.95811100 & -0.00015000 \\ 4.70755300 & -1.16676000 & 1.19522800 \\ 4.70882800 & -1.16455900 & -1.19515400 \\ 6.05724500 & -1.54102100 & 1.20714300 \\ 4.19165700 & -1.04025300 & 2.14886000 \\ 6.05853200 & -1.53878300 & -1.20631700 \\ 4.19395900 & -1.03630500 & -2.14911000 \\ 6.74137300 & -1.72730200 & 0.00060800 \\ 6.57432300 & -1.69457400 & 2.15393500 \\ 6.57662900 & -1.69057400 & -2.15283500 \\ 7.78908400 & -2.02255900 & 0.00089400 \\ 0.27023500 & 0.40017400 & -0.00061300 \\ -0.88321700 & -0.25404500 & -0.00091100 \\ -0.44528300 & 3.17080400 & 0.00032800 \\ -0.91333500 & -1.52156600 & -0.00183200 \\ -1.96370300 & 0.48557800 & -0.00018200 \\ -3.77873800 & -1.92662500 & 0.00014200\end{array}$

\section{Cs-2}

\begin{tabular}{|c|c|c|}
\hline \multicolumn{2}{|c|}{ Sum of electronic and zero-point Energies= } & -209.094527 \\
\hline \multicolumn{2}{|l|}{ Sum of electronic and thermal Energies $=$} & -209.089701 \\
\hline \multicolumn{2}{|c|}{ Sum of electronic and thermal Enthalpies= } & -209.088756 \\
\hline \multicolumn{2}{|c|}{ Sum of electronic and thermal Free Energies $=$} & -209.124885 \\
\hline Electronic Energy in gas phase $=$ & & -209.1163625 \\
\hline Electronic Energy in acetonitrile= & & -209.1602134 \\
\hline 2.45246975 & 0.00096706 & 0.00022320 \\
\hline 3.56728879 & 0.00070968 & 0.00105075 \\
\hline
\end{tabular}


$\mathrm{O}$

O

Cs

$\begin{array}{ccc}1.89067740 & -1.13301756 & -0.00020610 \\ 1.88731788 & 1.13337624 & -0.00020624 \\ -0.88192854 & -0.00017057 & 0.00001652\end{array}$




\section{Reference:}

(1) a) Riduan, S. N.; Zhang, Y.; Ying, J. Y. Angew. Chem. Int. Ed. 2009, 48, 3322-3325; b) Motokura, K.; Kashiwame, D.; Miyaji, A.; Baba, T. Org. Lett. 2012, 14, 2642-2645; c) Motokura, K.; Kashiwame, D.; Takahashi, N.; Miyaji, A.; Baba, T. Chem. Eur. J. 2013, 19, 10030-10037.

(2) Chong, C. C.; Kinjo, R. Angew. Chem., Int. Ed. 2015, 54, 12116-12120.

(3) Sarvari, M. H.; Sharghi, H. J. Org. Chem. 2006, 71, 6652-6654.

(4) Cui, X.; Zhang,Y.; Deng, Y.; Shi, F. Chem. Commun. 2014, 50, 189-191.

(5) Zhang, L.; Han, Z.; Zhao, X.; Wang, Z.; Ding, K. Angew. Chem., Int. Ed. 2015, 54, 6186-6189.

(6) Song, R. -J.; Liu, Y.; Hu, R. -X.; Liu, Y. -Y.; Wu, J. -C.; Yang, X. -H.; Li, J. -H. Adv. Synth. Catal. 2011, 353, 1467-1473.

(7) Wolfgang, M.; Dieter, M.; Lothar, Z.; Guenter, R.; Ernst, S.; Marita, S. Ger(East). 1984, DD214125 A1 19841003.

(8) Zhang, C.; Xu, Z. -J.; Shen, T.; Wu, G. -L.; Zhang, L. -R.; Jiao, N. Org. Lett. 2012, $14,2362-2365$.

(9) Lebleu, T.; Kotsuki, H.; Maddaluno, J.; Legros, J. Tetrahedron Lett. 2014, 55, 362-364.

(10) Fu, R.; Yang, Y.; Chen, Z.; Lai, W.; Ma, Y.; Wang, Q.; Yuan, R. Tetrahedron 2014, 70, 9492-9499.

(11) Gu, D. -W.; Guo, X. -X. Tetrahedron 2015, 71, 9117-9122.

(12) Perillo, I.; Caterina, M. C.; Lopez, J.; Salerno, A. Synthesis 2004, 6, 851-856.

(13) Jacquet, O.; Gomes, C. Das Neves; Ephritikhine, M.; T. Cantat, J. Am. Chem. Soc. 2012, 134, 2934-2937.

(14) Wolfgang, W.; Patrick, D. J. Chem. Res. Miniprint 1980, 11, 4513-4521.

(15) Bouchet, P.; Jaquier, R.; Pereillo, J. -M.; Elguero, J. Soc. Chim. Fr. 1972, 2264-2271.

(16) Wang, D.; Kuang, D.; Zhang, F.; Yang, C.; Zhu, X. Adv. Synth. Catal. 2015, 357, 714-718.

(17) Yang, Z.; Yu, B.; Zhang, H.; Zhao, Y.; Ji, G.; Ma, Z.; Gao, X.; Liu, Z. Green $\mathrm{S} 63$ 
Chem. 2015, 17, 4189-4193.

(18) Fu, M.; Shang, R.; Cheng, W.; Fu, Y. Angew. Chem., Int. Ed. 2015, 54, 9042-9046.

(19) Sueki, S.; Kuninobu, Y. Org. Lett. 2013, 15, 1544-1547.

(20) Santoro, O.; Lazreg, F.; Minenkov, Y.; Cavallo, L.; Cazin, C. S. J. Dalton Trans. 2015, 44, 18138-18144.

(21) Samples, M. S.; Yoder, C. H. J. Organomet. Chem. 1986, 312, 149-153.

(22) Besenius, P.; Cormack, P. A. G.; Ludlow, R. F.; Otto, S.; Sherrington, D. C. Chem. Commun. 2008, 2809-2811.

(23) S. Das, F. D. Bobbink, G. Laurenczy, P. J. Dyson, Angew. Chem., Int. Ed. 2014, 53, 12876-12879.

(24) Gaussian 09, Revision B.01, Frisch, M. J.; Trucks, G. W.; Schlegel, H. B.; Scuseria, G. E.; Robb, M. A.; Cheeseman, J. R.; Scalmani, G.; Barone, V.; Mennucci, B.; Petersson, G. A.; Nakatsuji, H.; Caricato, M.; Li, X.; Hratchian, H. P.; Izmaylov, A. F.; Bloino, J.; Zheng, G.; Sonnenberg, J. L.; Hada, M.; Ehara, M.; Toyota, K.; Fukuda, R.; Hasegawa, J.; Ishida, M.; Nakajima, T.; Honda, Y.; Kitao, O.; Nakai, H.; Vreven, T.; Montgomery, J. A.; Peralta, Jr., J. E.; Ogliaro, F.; Bearpark, M.; Heyd, J. J.; Brothers, E.; Kudin, K. N.; Staroverov, V. N.; Keith, T.; Kobayashi, R.; Normand, J.; Raghavachari, K.; Rendell, A.; Burant, J. C.; Iyengar, S. S.; Tomasi, J.; Cossi, M.; Rega, N.; Millam, J. M.; Klene, M.; Knox, J. E.; Cross, J. B.; Bakken, V.; Adamo, C.; Jaramillo, J.; Gomperts, R.; Stratmann,R. E.; Yazyev, O.; Austin, A. J.; Cammi, R.; Pomelli, C.; Ochterski, J. W.; Martin, R. L.; Morokuma, K.; Zakrzewski, V. G.; Voth, G. A.; Salvador, P.; Dannenberg, J. J.; Dapprich, S.; Daniels, A. D.; Farkas, O.; Foresman, J. B.; Ortiz, J. V.; Cioslowski, J.; Fox, D. J. Gaussian, Inc., Wallingford CT, 2010 . 